\title{
Focal Adhesion Kinase Signaling Spatially Regulates Adhesion Dynamics in
} Fibroblasts.

\author{
Marcin.P Iwanicki
}

Nysa, Poland

\section{B.S George Mason University}

Fairfax, Virginia

A Dissertation presented to the Graduate Faculty of the University of Virginia in Candidacy for the Degree of

Doctor of Philosophy

Department of Microbiology

University of Virginia

May 2008

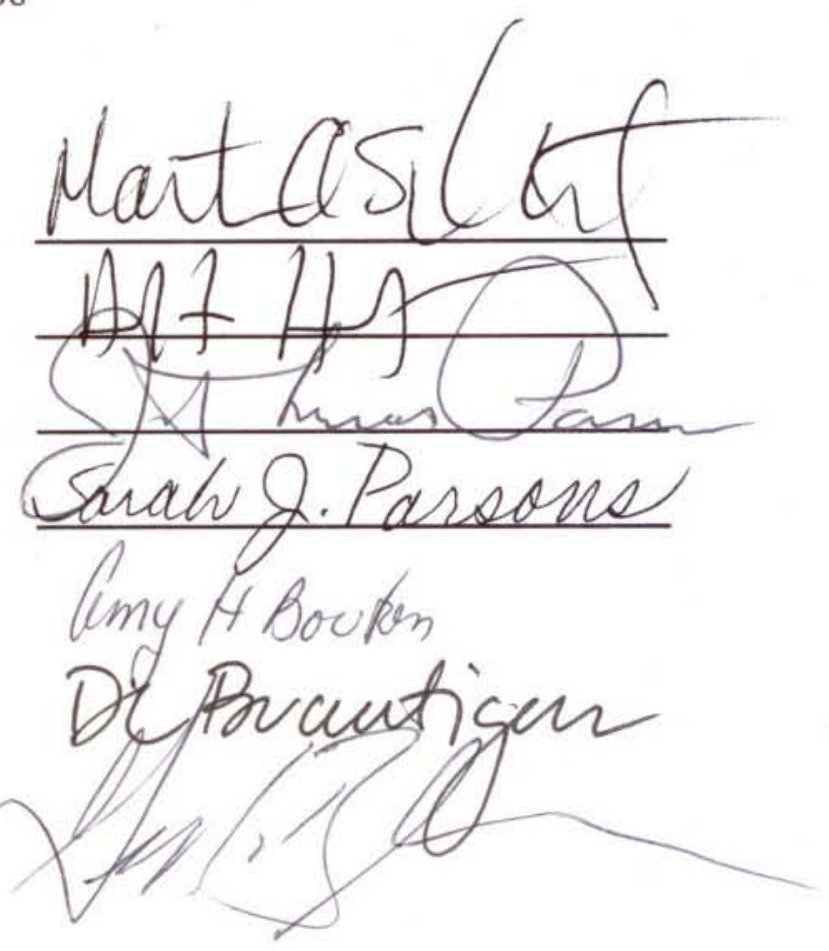


I dedicate this thesis to all my teachers. 


\section{Context}

Table of Context

List of abbreviations iii

$\begin{array}{ll}\text { Abstract } & 1\end{array}$

$\begin{array}{lr}\text { Chapter } 1 & 3\end{array}$

$\begin{array}{ll}\text { Introduction } & 3\end{array}$

Why study cell migration $\quad 3$

$\begin{array}{ll}\text { Cell migration Overview } & 4\end{array}$

ECM components $\quad 6$

$\begin{array}{ll}\text { ECM receptors } & 8\end{array}$

$\begin{array}{ll}\text { Integrin mediated adhesions } & 10\end{array}$

$\begin{array}{ll}\text { Cytoskeleton and cell migration } & 10\end{array}$

Dynamic regulation of adhesion $\quad 14$

$\begin{array}{ll}\text { Adhesion receptor signaling } & 17\end{array}$

$\begin{array}{lr}\text { Soluble factor receptor activation } & 19\end{array}$

Rho GTPase activation and cytoskeleton 21

Signaling and cell migration $\quad 26$

Cancer cell migration $\quad 28$

$\begin{array}{ll}\text { Src tyrosine kinase } & 31\end{array}$

FAK structure and regulation 33

FAK localization in cells $\quad 35$

FAK signaling $\quad 35$

Genetic analysis of FAK function 37

$\begin{array}{ll}\text { FAK and cancer progression } & 40\end{array}$

$\begin{array}{ll}\text { Figures } & 42\end{array}$

$\begin{array}{lr}\text { Dissertation focus } & 58\end{array}$ 
FAK couples Rho signaling to adhesion movement and trailing edge retraction in fibroblast

Introduction

Materials and methods

Results

Discussion

Figures and Tables

RACK 1 organizes FAK-induced ERK activation and localization to adhesions

Introduction

Materials and methods

Results

Discussion

Figures

Summary and possible models

Role of FAK in trailing edge retraction and adhesion movement

Role of RACK 1 in the regulation of FAK/ERK dependent adhesion disassembly in the lamellipodia of migrating cells

FAK couples two distinct signaling pathways to spatially regulate adhesion dynamics

Figures 


\section{List of Abbreviations}

ACF

ADF

ADP

Arp

ARPC

ASAP

ATP

Cas

CAT

Cdc42

CLASP

CRD

DAD

Dbl

DH

Dia

DID

ECM

EGF

EGFR

EMT

Ena/Vasp

ERK

FAK
Actin microtubule linker protein

Actin depolymerizing factor

Adenosie diphosphate

Actin related protein

Actin related protein complex

Arf GTPase activating protein

Adenosine triphosphate

Crk associated substrate

Collective amoeboid transition

cell division cycle GTP binding protein 42

Cytoplasmic linker associated protein

Cystine rich domain

Dia autoregulatory domain

Diffuse-B-cell lymphoma

Dbl homology

Diaphanous

Dia inhibitory domain

Extracellular matrix

Epidermal growth factor

Epidermal growth factor receptor

Epithelial mesenchyma transition

Enabled/Vasolidator-stimulated phosphoprotein

Extracellular regulated kinase

Focal adhesion kinase 
FERM

FH

fMLP

FRT

GAP

GDI

GEF

GFP

GRAF

Grb

GTP

HEK

HUVEC

ILK

IRSp

JAK-

JMR

LPA

MAPK

MEK

MHC

MLC

MLCK

MLCP

MP

NM erythrocyte band four.1-ezrin-radixin-moesin

Formin homology

formyl-methionyl leucine-phenylalanine

flp recombinase target

Guanine activating protein

Guanine dissociation inhibitor

Guanine exchange factor

Green fluorescent protein

GTPase regulator associated with FAK

Growth factor receptor binding protein

Guanine triphosphate

Human embryonic kidney

Human umbilical endothelial cells

Integrin linked kinase

Insulin receptor substrate protein

Janus kinase

Juxtamembrane

Lysophosphatidic Acid

Mitogen activated protein kinase

MAP kinase/ERK kinase

Myosin heavy chain

Myosin light chain

Myosin light chain kinase

Myosin light chain phosphotase

MAP kinase partner

Non muscle myosin 
PAK

PDGF

PDGFR

PDZ

PH

PI3K

PIP2

PIP3

PKC

PTX

PYK

Rac

RACK

Raf

RBD

RFP

Rho

RhoGEF

RNAi

ROCK

SH2

SH3

Src

WASP p21 activated kinase

Platelet derived growth factor

Platelet derived growth factor receptor PSD95/dlg/ZO-1 domain Pleckstrin homology Phosphoinositol kinase 3

Phosphoinositol 4,5 phosphate

Phosphoinositol 3,4,5 phosphate

Protein kinase C Pertussis toxin

Protein tyrosine kinase

Ras related protein

Receptor of activated protein kinase C

Ras activated factor

Rho binding domain

Red fluorescent protein

Ras homologous gene

Rho guanine nucleotide exchange factor

RNA interference

Rho coil-coil associated kinase

Src homology 2

Src homology 3

Sarcoma oncogene

Wiskot Aldrich syndrome protein 


\section{Abstract}

A key step in cell migration is the dynamic formation and disassembly of adhesions at the front and the concomitant movement and release of adhesions in the rear of the cell. In this thesis we present the evidence that the signals regulating adhesion dynamics in the rear of the cells differ from the signals that regulate adhesion dynamics in the front.

The first part of the work presented in this dissertation demonstrates that focal adhesion kinase (FAK) signaling to specific regulators of Rho GTPase controls adhesion movement and trailing edge retraction in fibroblasts. We present a model in which addition of Lysophosphatidic acid (LPA) induced the movement of adhesions and retraction of the trailing edge, thus mimicking tail retraction in migrating cells. FAK, guanine nucleotide exchange factors (GEFs) for Rho and the Rho effector, Rho Kinase II (ROCKII) are critical for the regulation of adhesion movement and trailing edge retraction. Down-regulation of FAK by small interfering RNAs (siRNAs) or small hairpin RNAs (shRNAs) blocked LPA induced adhesion movement and trailing edge retraction. This phenotype was rescued by ectopic expression of PDZ-RhoGEF or a Rho effector domain mutant that activates ROCK. The knockdown of PDZ-RhoGEF or ROCK II inhibited LPA-induced trailing edge retraction and adhesion movement. Moreover, over expressed PDZ-RhoGEF co-immunoprecipitated with FAK and localized to FAK containing adhesions. These studies support a model in which FAK and PDZRhoGEF co-operate to induce Rho/ROCKII-dependent adhesion movement and trailing edge retraction in response to LPA.

In the second part of this dissertation, we demonstrate the importance of a specific scaffold in targeting the MAP kinase pathway to adhesion structures found in the 
areas of active protrusions (front). We show that the RACK1 (Receptor Activated Kinase C) scaffold protein specifically functions in integrin-mediated activation of the MAPK/ERK cascade and targets active ERK to adhesions present in the lamellipodia of protruding cells. We found that RACK1 associates with the core kinases of the ERK pathway, Raf, MEK and ERK, and that attenuation of RACK1 expression resulted in a decreased ERK activity in response to adhesion. RACK1 silencing also caused a reduction of active ERK in adhesions and decreased the rate of adhesion disassembly in the areas of active protrusions. Our data further indicate that FAK is an upstream activator of the RACK1/ERK pathway in the regulation of adhesion disassembly in the lamellipodia. We suggest that RACK1 tethers the ERK pathway to adhesions found in the lamellipodia and channels signals from upstream activation by integrins to downstream targets at these sites.

In summary, the data presented in Chapters 2 and 3 argue that signaling pathways regulating adhesion dynamics within the leading edge of migrating cell differ from the signals that regulate adhesion dynamics at the trailing edge of the cell. We propose that FAK is a major component in coupling the MAP kinase pathway adhesion disassembly in the leading edge and Rho pathway to adhesion movement at the trailing edge. 


\section{CHAPTER 1}

Introduction

\section{Why study cell migration?}

The ability of cells to migrate is a fundamental biological phenomenon that is required for the persistence and survival of multicellular organisms (Horwitz and Webb, 2003). Cell migration starts from the moment of conception and continues throughout the life span of an organism. First, the fertilized egg has to migrate to the proper locale to prepare for the initial cell division. Second, in developing embryo primordial germ cells (precursors of gametes) migrate through the hindgut epithelium to the sites of gonad development (Laird et al., 2008). In adult organisms immune cells constantly survey tissues to look for potential pathogens (Cook and Bottomly, 2007). During injury (wound healing) stromal cells migrate to fill the injury site (Fuchs, 2007).

An alteration of the proper regulatory signals in migratory cells leads to pathological situations. In cancer, aberrant cell migration leads to the spread of cells to distant sites, resulting in dissemination of the cancer throughout the body (Wolf and Friedl, 2006). In muscular dystrophy, aberrant regulation of skeletal muscle stem cell migration leads to the failure of muscle repair following injury (Laird et al., 2008). Inflammation of arteries during atherosclerosis is induced in part by monocyte derived cells that fail to migrate out of the atherosclerotic lesions (Trogan et al., 2006). Therefore, understanding the biochemical and mechanical properties that regulate the migratory process is crucial for the development of therapeutics that may have useful applications in cancer progression, stem cell transplantation and inflammation associated with atherosclerosis. 
In the introduction to the dissertation, I cover the fundamental issues that are critical for cell migration. Following the overview of cell migration, I will introduce selected extracellular matrix (ECM) components on which cells migrate. I will also review the ECM receptors expressed on the cell surface that are needed for cellular migration. Next, I will focus on the importance of cell adhesion to ECM and cytoskeletal network in cell migration. Further, I will review current knowledge about selected receptor mediated signal transduction pathways that have been implicated in the regulation of adhesions, cytoskeleton and cell migration. Finally, I will describe the plasticity of migratory behaviors that cells can adopt in disease like cancer and discuss the pro migratory signaling pathways that will be the main theme of this work.

\section{Cell Migration an Overview}

Almost every aspect of life involves cell migration (Webb et al., 2005). During development, the blastocyst migrates as collective sheets to form three layers of the developing embryo (Anderson et al., 2000). Eventually cells within the developing embryo will migrate to form tissues and organs (Keller, 2005). In the developing cerebrum, neuronal stem cells migrate from the epithelial lining beneath the brain into the cerebrum, differentiate and extend axons to form synapses (Horwitz and Webb, 2003). Also the body plan of a vertebrate is entirely mediated by cell migration (Keller, 2006). For example, in the developing frog embryo the formation of a head and tail is achieved by polarized cell movement (Keller, 2002). These movements are known as convergence (to narrow the mediolateral aspect of the embryo) and extension (to elongate the anteriorposterior aspect of the embryo) of an embryo (Keller et al., 2000). Convergence/extension is achieved by increased mesoderm cell polarization followed by 
medial and lateral protrusive activities within gastrula (Wallingford et al., 2000; Wilson and Keller, 1991). As a result, cells begin to migrate laterally in an up and down direction producing neurula and finally a tadpole (Keller, 2002).

Cell migration is fundamental not only in embryo development but also in maintaining adult homeostasis. For instance, epidermal cells of the skin are continuously renewed by virtue of dermal "newcomers" migration (Fuchs, 2007). Immune system homeostasis almost entirely relies on the migratory properties of cells (Ridley et al., 2003). Cells of the innate immune system migrate to survey tissues and organs during and post infection (Cook and Bottomly, 2007). Migration of cells of the acquired and humoral arm of the immune system is required for proper $\mathrm{T}$ and $\mathrm{B}$ cell maturation and their antigen dependent activation (Allen et al., 2007). Wound repair relies on cell migration. Following injury and platelet dependent fibrinogen production, fibroblasts first move into the wound to form new stromal cell layers and epithelial cells follow to complete wound closure (Watterson et al., 2007).

Cell migration is a cyclical process which starts with the ability of the cell to sense external signals and convert these signals into the mechanics of lamellipodia protrusion and trailing edge retraction necessary for cell translocation (VicenteManzanares et al., 2005). Migratory cells are coupled to the extracellular matrix via cells' surface receptors localized within structures of close juxtaposition to the ECM (termed ECM adhesions). ECM adhesions connect the substrate on which the cell is migrating to the intracellular cytoskeleton. In the protruding lamellipodia, adhesions form and disassemble at relatively fast rates. In the trailing edge, adhesions tend to move inward and break during trailing edge retraction. In the following section, I will discuss the fundamental components of extracellular matrix that form the physical platform for cell migration. 


\section{ECM Components}

One of the most important cellular structures in biology are the sites of attachment of cells to basement membranes, attachment platforms rich in secreted extracellular matrix proteins. Basement membranes are present in the simplest multicellular sea creatures (for example, Coelenerates) as well as in the more complex and diverse higher mammals. The fundamental extracellular matrix proteins found in most basic basement membranes are type IV collagens and laminins (Hynes and Zhao, 2000). Both the fly and the worm have one pair of type IV collagen genes (Adams et al., 2000). In vertebrates there are three pairs of collagen IV genes that are arranged in an antiparallel (head to head) manner with a common promoter between them. Transcription of collagen IV in vertebrates results in production of heterotrimeric proteins containing two $\alpha 1$ subunits and one $\alpha 2$ subunit. Collagen IV molecules, through their triple alpha helical collagenous regions, interact with each other forming fibrils that play an essential role in the formation and maintenance of basement membranes (see Figure 1 A) (Kreis and Vale, 1999).

Laminins comprise another family of important extracellular matrix proteins. The laminins found in Drosphila and C.elegans are heterotrimers composed of one $\alpha$, one $\beta$ or one $\gamma$ subunit (Gotwals et al., 1994). In higher vertebrates the number of laminin subunits is far greater. In humans or mice there are five $\alpha$, three $\beta$ and three $\gamma$ subunits that make up sixteen different laminin heterotrimers (Aumailley et al., 2005). The sequence of the laminin $\alpha$ subunit contains a number of tandem repeats of epidermal growth factor like domains, G like domains ( sequences of approximately 200 residues that are repeated five times in the $\mathrm{C}$-terminal globular region of the laminin $\alpha$ subunit) and similarly $\mathrm{N}$-like domains that are placed at the $\mathrm{N}$ terminus of the molecule (Figure 
1B) (Colognato and Yurchenco, 2000). Similar to collagen IV, laminins play a central role in the formation and stability of basement membranes.

Fibronectin is an ECM protein that is not present in basement membranes but is essential for development. Fibronectin is a large glycoprotein composed of three different types of repeating modules, type I, type II and type III repeats (Figure 1C) (Pankov and Yamada, 2002; Potts and Campbell, 1996), (Hynes R.O, 1990). The type I repeat is composed of 45 amino acids and is found in the amino terminal and carboxy terminal regions of the protein (Bork et al., 1996). The amino terminal type I repeat facilitates interaction with other fibronectin molecules and collagen molecules and promotes formation of fibrils (Hohenester and Engel, 2002). Type II repeats are found within the $\mathrm{N}$-terminal portion of the molecule and are positioned along with type I repeats within a collagen binding region of fibronectin that also contributes to fibril formation (Morla and Ruoslahti, 1992). Type III repeats make up the most of the molecule (WierzbickaPatynowski and Schwarzbauer, 2003). In contrast to type I and type II repeats, type III repeats are encoded by different exons within the fibronectin gene (Schwarzbauer et al., 1983). Alternative splicing of type III repeat exons can generate various fibronectin molecules that are expressed differentially during the development of the organism (Schwarzbauer et al., 1983). Type III repeats facilitate the interaction of fibronectin with other fibronectin molecules as well as cell surface receptors and heparins (Hynes and Yamada, 1982). Mice lacking the fibronectin gene die from defects in vascular development (Astrof et al., 2007). Thus, fibronectin along with collagen IV and laminin are required for development.

The role of ECM proteins is to provide attachment sites for cells expressing ECM receptors and provide a platform for cell migration. In the following section I will describe the variety of extracellular matrix receptors that engage different substrates. 


\section{ECM Receptors}

The integrins are major cellular receptors for ECM and are well conserved among the phyla (Hynes and Zhao, 2000). Integrins are heterodimeric receptors (comprised of an $\alpha$ and $\beta$ subunit) that link components of the ECM to the cell cytoskeleton (Carrell et al., 1985). The $\alpha$ subunit noncovalently associates with $\beta$ subunit to form a heterodimer (Gailit and Ruoslahti, 1988). The integrin $\alpha$ and $\beta$ subunits are transmembrane glycoproteins with short cytoplasmic tails (Hynes, 1992). The $\mathrm{N}$ terminus of the $\alpha$ subunit contains the amino acid sequence (Asp-X-Asp-X-Asp-Gly-X-X-Asp) that supports divalent cation binding and is necessary for proper integrin function (Figure 2) (Kirchhofer et al., 1990). Common to all $\beta$ subunits is a four fold repeat of a cystine rich segment within the $\mathrm{N}$ terminal portion of the subunit. These repeats are thought to be important for ligand binding (Hynes, 1992). The carboxy terminal portions (cytoplasmic domains) of both subunits serve as docking sites for the cytoskeleton and cytoskeleton associated proteins (Liu et al., 2000b).

Integrins are found in organisms including sponges, corals, nematodes, echinoderms and mammals (Burke, 1999). The Drosophila genome encodes two $\beta$ subunits ( $\beta$ PS and $\beta v$ ) and five $\alpha$ subunits ( $\alpha$ PS1- $\alpha$ PS-5) (Gotwals et al., 1994). All five $\alpha$ subunits pair to form complexes with $\beta \mathrm{PS}$, it is not known if $\beta \mathrm{v}$ pairs with any of the $\alpha$ subunits (Hynes and Zhao, 2000). The integrins, $\alpha$ PS1 $\beta$ PS and $\alpha$ PS2 $\beta$ PS are the receptors for laminin and collagen (Gotwals et al., 1994; Zavortink et al., 1993). In vertebrates there are eighteen $\alpha$ subunits and eight $\beta$ subunits and the combinatorial complexity yields twenty-two distinct integrins (Hynes, 1996). More than a half of vertebrate $\alpha$ subunits contain an additional domain called the "I" domain (Hogg et al., 
1994). "I" domains are the major cation and ligand binding domains in integrins (Figure 2) (Nolte et al., 1999). I domains of $\alpha \mathrm{L}$ integrins have been shown to bind to ICAM molecules (Luo et al., 2007). In vertebrates $\alpha_{1-2}$ and $\alpha_{6-7}$ subunits can pair with $\beta_{1}$ subunit and yield receptors for collagen and laminin. Fibronectin is recognized by the following integrin dimers $\alpha_{3-5} \beta_{1}, \alpha_{V} \beta_{6} \alpha_{V} \beta_{3}, \alpha_{V} \beta_{1}, \alpha_{V} \beta_{5}$ and $\alpha_{4} \beta_{7}$ (Faull and Ginsberg, 1996). Genetic deletion of mouse integrin alpha subunits $\alpha_{3,4,5,6,8}$ and $\alpha_{\mathrm{v}}$ is embryonic lethal (GeorgesLabouesse et al., 1996; Kreidberg et al., 1996; Yang et al., 1993; Yang et al., 1995) (Hynes, 1996), whereas mice knocked out for $\alpha_{1}, \alpha_{I I b}$ and $\alpha_{E}$ are viable and fertile (Gardner et al., 1996) (Hynes, 1996), suggesting some functional redundancy. However, targeted deletion of $\alpha_{7}$ results in the development of muscular dystrophy (Mayer et al., 1997) .Similarly, phenotypes due to deletion of mouse integrin $\beta$ subunits differ. For instance, knock outs of $\beta 1$ and $\beta 4$ subunits are embryonic lethal (Fassler et al., 1995; van der Neut et al., 1996), whereas, knock outs of $\beta_{2,3,5,6,7}$ are dispensable for development (Hynes, 1996).

Integrins are a major family of transmembrane receptors that translate extracellular cues into biochemical signals that regulate a variety of cellular processes (Schwartz and Ingber, 1994). The ability of integrins to regulate cell behavior is a result of the fact that integrins constitute an essential bridge between ECM and the cellular microfilament network, thus providing structure as well as sensors for the microenvironment. In the following section I will describe the nature of integrin substrate adhesions. 


\section{Integrin mediated adhesions.}

Integrin engagement with extracellular matrix components results in the formation of structurally distinct matrix adhesions (Horwitz et al., 1986). Early studies in cultured chicken fibroblasts revealed that matrix adhesions occur as elongated small regions along the ventral plasma membrane (Abercrombie and Dunn, 1975). These regions were termed focal adhesions and/or focal complexes (I will use the term adhesions throughout the thesis) (Burridge et al., 1988; Burridge et al., 1987). In the earlier experiments, it was recognized that adhesions were associated with the actin cytoskeleton and provided structural support for the attached cell (Burridge et al., 1987). In addition to the association of actin with adhesions, immunofluorescence experiments revealed the multi-protein nature of adhesions, containing transmembrane ECM receptors (integrins), lipid kinases/ phosphatases and non-enzymatic scaffold proteins (Zaidel-Bar et al., 2003). Because of the direct link to the cell cytoskeleton, adhesions are part of the cytoskeletal mechanics that drive cell migration. In the following chapter, I will focus on the role of cytoskeleton and selected associated proteins with the cytoskeleton in the regulation of cell migration.

\section{Cytoskeleton and cell migration.}

Cellular migration is a mechanical process that involves the temporal and spatial interplay amongst components of the ECM, cellular adhesion and cytoskeleton. The initial event of polarized movement is the formation of a predominant membrane protrusion (lamellipodium or filopodia) which is driven by the actin cytoskeleton (Euteneuer and Schliwa, 1984; Pollard and Borisy, 2003; Verkhovsky et al., 1999). Actin 
filaments are assembled from actin monomers (Drenckhahn and Pollard, 1986). Actin filaments within the protrusion are polarized in such a way that barbed ends (fast assembling) face the protrusion and pointed ends (slow assembling) face distally from the protrusion (Borisy and Svitkina, 2000). Organization of actin in the lamellipodium resembles a branching dendritic meshwork (Svitkina and Borisy, 1999), whereas in filopodia actin is organized in bundles (Schafer et al., 1998; Welch and Mullins, 2002). In lamellipodia, actin polymerization is catalyzed by components of the actin related protein complex Arp2 and Arp3 (Arp2/3). The Arp2/3 complex contains seven associated subunits that include Arp2, Arp 3 and ARPC1-5 (Machesky et al., 1994). Purified Arp2/3 can nucleate actin polymerization in vitro and cross-link (arrange) newly formed filaments into Y - branched arrays (Mullins et al., 1998). In lamellipodia, Arp2/3 induces the formation of new actin filaments that branch off already existing filaments (Pollard and Beltzner, 2002). In filopodia actin polymerization is also driven by Arp2/3-dependent mechanisms that promote actin filament barbed end assembly that pushes the membrane out (Miki et al., 1998). In addition filopodia can also be generated via Arp2/3 independent mechanisms, involving the formin -dependent barbed end polymerization and membrane extension (Pellegrin and Mellor, 2005).

The rate of actin polymerization in lamellipodia and filopodia is mainly controlled by actin-binding proteins that can directly promote or prevent polymerization of actin, disrupt existing actin filaments or stabilize actin filaments. For instance, profilin is an actin binding protein that can either promote or prevent actin polymerization (Kaiser et al., 1999). It promotes actin filament polymerization by targeting actin monomers to barbed ends of the filaments (Goldschmidt-Clermont et al., 1991). Profilin also prevents actin polymerization by decreasing the free monomer concentration available for spontaneous filament nucleation (Pollard and Borisy, 2003). Thymosin $\beta 4$ is an actin 
binding protein that can regulate actin dynamics (Cassimeris et al., 1992). Contrary to profiling, thymosin $\beta 4$ will not target actin monomers to barbed ends of polymerizing filaments (Goldschmidt-Clermont et al., 1992). Thus, it suppresses barbed end elongation by decreasing the pool of available actin monomers.

Another protein that can influence actin polymerization in the lamellipodium is the actin destabilizing factor ADF/cofilin (Ghosh et al., 2004). ADF/Cofilin is an actin severing molecule that cuts "old actin" filaments and creates free barbed ends for further polymerization, thus, it contributes to actin filament turnover (Andrianantoandro and Pollard, 2006). Studies in mammary adenocarcinoma cells revealed that lack of cofilin results in stabilization of actin filaments that induces persistence of lamellipodia (Sidani et al., 2007), supporting a role for cofilin in the turnover of pre-existing actin filaments.

Yet another mechanism for regulating actin dynamics within the lamellipodium is actin filament capping (Pollard and Borisy, 2003). Gelsolin is a protein that first cuts actin filaments and then caps one end of the filament (Kwiatkowski, 1999). This property of gelsolin has been attributed to its increased barbed end formation and stabilization of a polymerizing filament at the same time (Barkalow et al., 1996). Gelsolin null fibroblasts show increased actin bundling and decreased lamellipodia protrusions, probably due to decreased actin polymerization (Azuma et al., 1998).

In filopodial protrusions, another set of proteins has been found to regulate actin dynamics. Ena/Vasp proteins localize to the tips of barbed ends and antagonize capping and branching, thus promoting filament polymerization and stabilization (Krause et al., 2003). Mice lacking Ena/Vasp proteins are viable but show defects in brain development, and their neurons are severely depleted in filopodia (Kwiatkowski et al., 2007; Lebrand et al., 2004). Thus, Ena/Vasp plays a critical role in stabilization of polymerizing actin filaments that contribute to the formation of filopodia. 
Another component of the cytoskeleton that regulates actin dynamics during cell migration is myosin (Vicente-Manzanares et al., 2007). The myosins are actin-based molecular motors that can pull and bundle actin filaments (Bresnick, 1999). The myosin II family includes skeletal, cardiac and smooth muscle myosin as well as non muscle myosin (NM) (Conti and Adelstein, 2008). Every myosin II molecule is a hexamer composed of $\underline{\text { Myosin }} \underline{\text { Heavy }} \underline{\text { Chain dimers }}$ (MHC) and two pairs of regulatory Myosin $\underline{\text { Light }} \underline{\text { Chains }}$ (MLC). The MLCs are tightly bound to MHCs and play a role in stabilizing and regulating myosin structure (Amano et al., 1996a). The N-terminus of MHC is a globular head that contains ATPase activity and binds actin. The C-terminus of MHC is involved in myosin filament formation (Conti and Adelstein, 2008)(Figure 3). This arrangement of myosin allows for the actin filament to be translocated via the globular motor domain and to be maintained under tension via C-terminus association with other MHC molecules (cross-linking). NM II has been implicated in the mechanics of lamellipodial adhesion assembly, maturation and disassembly (Vicente-Manzanares et al., 2007) .

In addition to actin, microtubules have been implicated in regulation of migration in multiple cell types (Gundersen et al., 1998). Microtubules are comprised of tubulin dimmers $(\alpha$ and $\beta$ ) that derive the energy for polymerization from the sustained binding of guanine tri-phosphates (GTP) to the $\beta$ subunit (Howard and Hyman, 2003; Mitchison and Kirschner, 1984). Microtubule polymerization is favored when there is an abundance of GTP loaded dimers near the tips of microtubules (Howard and Hyman, 2003).

The regulation of microtubule stability is an important component of cell migration (Palazzo et al., 2004). In polarized fibroblasts, tips of stable (i.e., the rate of polymerization greatly exceeds the depolymerization rate) microtubules are predominantly found in protruding lamellipodia (Kaverina et al., 1999), as shown by total 
internal reflection microscopy (TIR-FM). It has been proposed that the role of stable microtubule penetration into lamelliopodia is to deliver lipid vesicles containing regulatory molecules (Lin et al., 2002).

Genetic attenuation of expression of microtubule tip binding proteins has been shown to perturb the stability of microtubules and the polarization of migratory

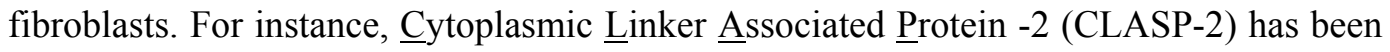
shown to be necessary for maintenance of stable microtubules in fibroblast lamellipodia (Drabek et al., 2006). Cells lacking CLASP-2 show multiple short lived lamellipodia and lose directional migration (Drabek et al., 2006). Another molecule that has been implicated in the regulation of microtubule dynamics within the lamellipodium is actinmicrotubule linker protein ACF-7. Similar to CLASP-2 null fibroblasts, ACF-7 depleted cells have reduced levels of stable microtubules and fail to maintain directional migration (Kodama et al., 2003). In summary microtubules along with actin filaments are essential to drive polarized cell migration.

In previous paragraphs, I introduced the basic knowledge about components (substrate, adhesions and cytoskeleton, see Figure 4A) that contribute to the migratory process. Cell matrix adhesions along with the cytoskeleton are dynamic and undergo constant remodeling during attachment and cell migration. In the following paragraphs I will describe how adhesion dynamics are regulated during migration.

\section{Dynamic Regulation of Adhesion}

Integrin-mediated adhesions are dynamic with defined lifespans (Webb et al., 2002). In the late 1970s, interference reflection microscopy was used to show that adhesions in chicken fibroblasts undergo dynamic changes during cell migration (Heath 
and Dunn, 1978). From these studies, it was inferred that adhesion dynamics were regulated by the actomyosin network that directly connected to these adhesions. Twenty years later, researchers using the $\beta 1$ integrin cytoplasmic domain fused to green fluorescent protein (GFP) observed that in stationary fibroblasts most of the integrin adhesions were highly mobile and tended to move along actin bundles towards the center of the cell. On the other hand, in actively moving cells, adhesions displayed polarized behavior. In the front of the cell adhesions were stationary with high frequency of disassembly whereas in the back of the cells, adhesions were elongated and moved along with the stress fibers towards the center of cell (Palecek et al., 1996; Smilenov et al., 1999). The movement of the elongated adhesions appear to result from the addition of adhesion molecules (e.g., vinculin, paxillin) at one end (assembly), and the removal of adhesion molecules at the other end (disassembly) of the adhesions - a process referred to as adhesion treadmilling (Digman et al., 2008) (Figure 4B). It is likely that changes in actomyosin contractility promote treadmilling of adhesions in the trailing edge (Digman et al., 2008).

Spatial difference in the adhesions dynamics (stationary adhesions vs treadmilling adhesions) is likely to involve regulation of myosins. In fibroblasts siRNAdependent attenuation of non-muscle myosin IIA/B expression resulted in impaired lamellipodial and trailing edge adhesion dynamics (Vicente-Manzanares et al., 2007). Therefore, it is likely that signaling events that impact myosins are crucial in the spatial regulation of adhesion dynamics.

Microtubules have been shown to "target "adhesion sites and induce adhesion disassembly (Kaverina et al., 1999). Using TIRFM, researchers showed that tips of microtubules are able to target adhesion sites and induce their disassembly. The pharmacological disruption of microtubules results in adhesion growth, whereas the 
removal of the inhibitor restores polymerization of microtubules and induces adhesion turnover (Ezratty et al., 2005). These observations suggested the hypothesis that protein trafficking associated with polymerizing microtubules could contribute to adhesion turnover. Caveolin have been shown to be delivered by microtubules to the plasma membrane (Mundy et al., 2002) and localized to adhesion sites (del Pozo et al., 2005). Interestingly, caveolin null fibroblasts have increased number of focal adhesions and their dynamics is impaired (Grande-Garcia et al., 2007). These data further suggested the hypothesis that microtubule-dependent delivery of caveolin is necessary for adhesion turnover. However, more experiments need to be done to fully explore this hypothesis.

Cellular proteases have been implicated in the regulation of adhesion dynamics. Early studies showed that inhibition of the calcium-dependent protease calpain reduces rates of cell migration, induces growth of adhesions and prevents trailing edge retraction (Huttenlocher et al., 1997). The likely mechanism for calpain-mediated regulation of adhesion is proteolytic degradation of adhesion components including FAK (Carragher et al., 2003) and talin (Franco et al., 2004).

In summary, substrate adhesions are dynamic entities that undergo constant remodeling during cell migration. The differences in their spatial behavior are most likely due to localized coupling of distinct signaling pathways. In the following paragraphs, I will introduce the initiation of signaling pathways from selected plasma membrane receptors that have been implicated in the control of adhesion dynamics and cell $\underline{\text { migration. }}$ 


\section{Adhesion Receptor Signaling.}

Abundant evidence exists indicating that integrin-mediated adhesions transmit signals from the outside of the cell to the inside as well as integrate signals generated by growth factor receptors (Schwartz, 2001). Evidence that integrin-mediated adhesions direct signals into the cells comes from experiments showing that integrin-dependent platelet aggregation induces tyrosine phosphorylation of several proteins in the range of $80-130 \mathrm{kDa}$ (Shattil and Brugge, 1991). In related experiments, adhesion of carcinoma cells or fibroblasts to fibronectin induced the tyrosine phosphorylation of similar sized proteins (Guan et al., 1991). Interestingly, the same set of proteins that were predominantly tyrosine phosphorylated in response to integrin mediated adhesion were found to be tyrosine phosphorylated in cells transformed with oncogenic Src (Sarcoma) kinase or in cells stimulated with soluble growth factors (Parsons and Parsons, 2004). These observations indicated that integrin-mediated adhesions induced intracellular signals in a similar fashion to already known signal transducers.

Another example of adhesion-mediated signaling is cytoplasmic alkalization (Hynes, 1992). In various cell types (e.g fibroblast, leukocytes, endothelial cells) integrinmediated adhesion to fibronectin has been shown to induce changes in cytoplasmic $\mathrm{pH}$ that correlates with the ability of cells to spread on matrix proteins (Ingber et al., 1990). The mechanism of integrin control of intracellular $\mathrm{pH}$ involves microclustering and membrane immobilization of $\alpha 5 \beta 1$ integrin and subsequent opening/activation of a sodium /hydrogen $\left(\mathrm{Na} / \mathrm{H}^{+}\right)$antiporter (Schwartz et al., 1991). In some cases the activation of the $\mathrm{Na}+/ \mathrm{H}+$ antiporter is mediated by integrin-dependent augmentation of growth factor induced signaling to Protein Kinase C (Schwartz and Lechene, 1992). These 
experiments showed that the pathways by which integrins transduce signals can synergize with growth factor receptor initiated pathways to regulate cellular alkalization.

The activation of integrins and transduction of adhesion-dependent signals involves many steps. First, prior to ligand binding to integrins, intermolecular interactions between cytoplasmic tails of $\alpha$ and $\beta$ subunits appear to be important in "priming" the affinity of integrin extracellular domains for the ligand (Hughes et al., 1996). The interaction between cytoplasmic tails of $\alpha$ and $\beta$ subunits depends on the conformational rearrangements mediated by the membrane spanning region of each integrin subunit (Du et al., 1993).

Second, integrin ligation to extracellular matrix components (e.g fibronectin) further induces conformational changes (activation of integrins) that are propagated to the transmembrane and cytoplasmic domain of integrins in an allosteric manner to induce $\alpha \beta$ dimer formation (Liddington and Ginsberg, 2002).

Third, integrin oligomerization induces the recruitment of downstream effectors via interactions with the cytoplasmic tails of $\alpha$ and $\beta$ subunits (Brunton et al., 2004). The cytoplasmic tails of $\beta$ subunits have been shown to mediate stable interactions with a greater number of partners than the cytoplasmic tails of $\alpha$ subunits (Liu et al., 2000a). The $\beta$ subunit cytoplasmic tail associates with an array of different proteins, including the actin binding proteins, talin, filamin, $\alpha$-actinin, and myosin (Horwitz et al., 1986; Jenkins et al., 1998; Otey et al., 1990; Pavalko et al., 1989), the scaffolding proteins, paxillin, Grb2, Rack 1 (Law et al., 1996; Liliental and Chang, 1998; Schaller et al., 1995) and the protein kinases, integrin linked kinase (ILK) and FAK (Hannigan et al., 1996) (Schaller et al., 1995).

Fourth, integrin oligomerization leads to activation of ILK and FAK. Integrininitiated signaling and further signal propagation to integrin binding partners has been 
shown to be important in many aspects of cell biology including cell migration. (Schwartz, 1997; Schwartz, 2001).

Integrin activation initiates signaling pathways that support membrane protrusions (Schwartz and Ginsberg, 2002). During cell migration, activated integrins are found predominantly at sites of lamellipodial protrusions (Kiosses et al., 2001). Integrins via their ability to initiate the formation of adhesions and localize signaling to the cytoskeleton are the main plasma membrane receptors that regulate protrusion formation and adhesion dynamics in polarized cell migration (Ridley et al., 2003). However, growth factor receptor activation has been shown to mediate pro - migratory signals. In the following paragraph, I will describe activation of selected soluble factor receptors that are important to consider when studying cell migration.

\section{Soluble Factor Receptor Activation.}

Cells plated on ECM components in the absence of soluble mitogenic factors initiate lamellipodia protrusions but fail to polarize and migrate (Schwartz and Ginsberg, 2002). These experiments indicate that soluble factors support polarization and cell migration. Among the vast array of cell surface soluble factor receptors, Lysophosphatidic Acid (LPA), and Platelet Derived Growth Factor (PDGF) receptors are relevant to this work. Therefore, I will focus my attention on these receptors.

LPA receptors belong to a family of Edg receptors. Edg receptors are G proteincoupled receptors. LPA can recognize four different Edg receptors (edg 1-4). Edg 1 receptor is the most widely expressed receptor in the body (Mills and Moolenaar, 2003). Fibroblasts isolated from Edg 1 receptor null mice showed decreased but not abrogated 
migration in response to LPA, suggesting some redundancy among Edg receptors (Contos et al., 2000).

LPA binding to Edg receptors activates heterotrimeric guanine nucleotide binding regulatory proteins (G proteins) (Rozengurt, 2007). G proteins are composed of $\alpha, \beta, \gamma$ subunits. $\mathrm{G}$ proteins are classified into $\mathrm{Gs}, \mathrm{Gi}, \mathrm{Gq}$ and $\mathrm{G}_{12 / 13}$ (Rozengurt, 2007). LPA has been shown to couple via Edg receptors to $\mathrm{Gq}$, Gi and $\mathrm{G}_{12 / 13}$ (Mills and Moolenaar, 2003).

The activation of $\mathrm{G}$ proteins by agonist starts with binding of the cytoplasmic loop of an agonist receptor to a GDP-loaded $\alpha$ subunit of the G protein. As a result agonist-bound receptor provokes the exchange of GDP for GTP within the G protein $\alpha$ subunit. This reaction induces dissociation of the $\alpha$ subunit from the $\beta \gamma$ subunit and promotes both $\alpha$ and $\beta \gamma$-dependent downstream interactions. The system returns to the native state by the hydrolysis of GTP by the $\alpha$ subunit (Johnston and Siderovski, 2007).

There are two isoforms of PDGFR, $\alpha$ and $\beta$ (Ullrich and Schlessinger, 1990). Both isoforms are composed of extracellular ligand binding domains, transmembrane domains, juxtamembrane domains, protein tyrosine kinase domains and $\mathrm{C}$ terminal tail (Dibb et al., 2004). The ligand binding domain is composed of five immunoglobulin like repeats (Ullrich and Schlessinger, 1990). The transmembrane domain anchors the receptor within the plasma membrane, thereby connecting the extracellular environment with the internal compartment of the cell. The juxtamembrane (JMR) domain serves as an autoinhibitory domain in PDGFR kinase activation. Agonist binding promotes the kinase domain-catalyzed tyrosine phophorylation on tyrosine residues within the receptor, thereby activating the PDGFR (Dibb et al., 2004).

Upon ligand binding to the extracellular domain, PDGFR dimerizes to form $\alpha \alpha$, $\beta \beta$ or $\alpha \beta$ PDGFR dimers. Dimerization partially activates the kinase domain leading to 
autophosphorylation of a critical tyrosine residue (Y-857) within the kinase domain. Following this partial activation, the JMR unlocks the catalytic activity of the receptor which promotes further phosphorylation of residues within the kinase domain, the JMR and the C terminal tail(Johnson et al., 1996). Tyrosine phosphorylation within these domains creates high affinity binding sites for tyrosine rich recognition sites containing (Src homology-2) proteins (Dibb et al., 2004)

Along with integrins, $\mathrm{G}$ proteins and tyrosine kinase receptors have been shown to coordinate polarized cell migration in various systems (Ridley et al., 2003). Their effect on migration is confined to the ability to regulate Ras homologus gene family of small GTPases (Rho GTPases) - the main drivers of cytoskeleton dynamics. In the following paragraph, I will focus on the activation of Rho-GTPases and their effect on the cytoskeleton.

\section{Rho GTPase activation and the cytoskeleton}

Rho family GTPases belong to the low molecular weight (21 KD) protein family (small G proteins) of Ras-like GTPases (Ridley and Hall, 1992). Rho GTPases are ubiquitously expressed, and there are 22 members in the family. They act as molecular switches to control signal transduction pathways initiated by matrix and soluble growth factor receptors (Schwartz, 2004). The primary function of Rho GTPases is to regulate cytoskeleton dynamics (Bishop and Hall, 2000). Activation of Rho GTPases depends on the cycling between GDP- and GTP- bound state of the Rho molecule. When Rho is bound to GTP it is considered activated. On the other hand, when GDP is bound to Rho, the molecule is non-functional. These two nucleotide-bound states of Rho are tightly controlled by accessory proteins like guanine dissociation inhibitors (GDI), guanine 
nucleotide exchange factors (GEF) and GTPase activating proteins (GAPs) (Rossman et al., 2005).

GDI binds the isoprenyl moiety of Rho and sequesters it from the plasma membrane, creating a cytoplasmic pool of Rho available for activation. GEFs have affinity for GDP-bound Rho and facilitate exchange of GDP to GTP, thereby activating Rho proteins. There are 80 known GEFs. Although, there is great structural diversity among various GEFs, one common theme is that most of them possess diffuse-B-cell lymphoma (Dbl) homology (DH) domains. The DH domain of GEFs is required for interaction with Rho and nucleotide exchange (Rossman et al., 2005). However, there are GEFs that do not have DH domains but instead utilize DOCK homology regions to activate Rho proteins (Brugnera et al., 2002; Meller et al., 2002) .

GAPs bind to GTP-loaded Rho, increase intrinsic enzymatic GTPase activity, and promote release of phosphate, thus, inactivating Rho. GDP-bound Rho is again sequestered by GDI from the plasma membrane to the cytosol, and the cycle repeats (Reuther et al., 2001).

Tissue culture studies using activated forms of Rho showed their involvement in the regulation of the actin cytoskeleton (Raftopoulou and Hall, 2004). For instance, the small G protein Rac, when overexpressed in fibroblastic cells, promotes formation of broad membrane protrusions. Rac-dependent induction of lamellipodial protrusions is ascribed to activation of the Arp2/3 complex that facilitates polymerization of actin within lamellipodia (Wells et al., 2004).

Another small $\mathrm{G}$ protein is Rho A. Overexpression of Rho A in fibroblasts promotes actin bundle and adhesion formation (Ridley and Hall, 1992). Rho A-mediated regulation of the actin cytoskeleton is mainly attributed to activation of Rho effectors including Rho-associated coil-coil kinase (ROCK) that regulates myosin-dependent actin 
contractility and the formin diaphanous Dia-dependent actin polymerization (Bishop and Hall, 2000).

Still another member of the small G proteins is cell division cycle GTP binding protein $42(\mathrm{Cdc} 42)$. Overexpression of $\mathrm{Cdc} 42$ in fibroblasts results in the predominant formation of filopodia (Ridley, 2001). Cdc42-dependent induction of filopodia is mainly mediated by its effector, Wiskot Aldrich Syndrome protein (WASP) (Welch and Mullins, 2002). However, recent studies indicated that filiopodia could be formed in the absence of Cdc42, suggesting other compensatory mechanisms (Czuchra et al., 2005).

Coordination of cell protrusions with adhesion formation and disassembly, movement and trailing edge retraction during migration could be achieved by spatially localized signaling to small $\mathrm{G}$ proteins. One possible way to focus globally initiated small $\mathrm{G}$ protein signaling to a specific cellular region is the utilization of guanine exchange factors that are locally concentrated. Among the family of Dbl containing proteins there are GEFs that receive rather general signals from plasma membrane receptors and localize these signals to various cellular locales.

The group of Rho GEFs that link receptor-coupled heterotrimeric G protein signaling with Rho activation can localize to different subcellular locations (Banerjee and Wedegaertner, 2004). P115 RhoGEF and the PDZ domain-containing Rho GEF (PDZRhoGEF) operate via the regulator of G-protein signaling homology sequence (RGS) which binds to $\mathrm{G} \alpha$ 12/13 and increases its nucleotide catalytic activity (Chikumi et al., 2004). Ga12/13 binding to the RGS domain of these GEFs promotes GEFs binding to Rho, nucleotide exchange activity within Rho and Rho activation (Rossman et al., 2005) (Figure 5). Although the ways of linking hetrotrimeric $G$ protein signaling to Rho activation are the same between these two GEFs, their subcellular localization suggests a differential focusing of the Rho A signal. 
Over-expressed PDZ-Rho GEF but not p115RhoGEF is localized to the actin cytoskeleton in epithelial (Banerjee and Wedegaertner, 2004) and neutrophil cells (Wong et al., 2007). In neutrophils, PDZ-RhoGEF is mainly found within actin and myosin structures present in the trailing edge, suggesting that Rho signals to actomyosin within the trailing edge of migrating neurtophils (Wong et al., 2007). On the contrary, p115 RhoGEF is predominantly localized to the plasma membrane (Banerjee and Wedegaertner, 2004). When p115 RhoGEF is overexpressed in polarized neutrophils, it mainly localizes to the protruding front edge, suggesting Rho signals are localized to components within the membrane protrusions (Francis et al., 2006). It is clear from these experiments that signals imitated by the same set of $G$ proteins can diverge to different cellular locations and this depends on specific GEF utilization.

GEF molecules can couple Rho signaling to distinct cellular locales, however, another set of Rho signaling proteins is required for Rho dependent cytoskeleton regulation. The immediate effectors of Rho signaling are the main bridges between Rho GTPase activation and cytoskeleton remodeling (Bishop and Hall, 2000). For instance, RhoA-mediated regulation of the actin cytoskeleton is ascribed to two effector molecules, ROCK and Dia (Schwartz, 2004).

ROCK belongs to the family of serine threonine kinases. The ROCK molecule contains a N-terminal kinase domain, the Rho binding domain (RBD) the pleckstrin homology domain (PH) and a cystine-rich region (CRD) (Leung et al., 1995) (Figure 6). In the inactive state, the C-terminal RBD, the $\mathrm{PH}$ and the CRD of ROCK are folded over and bound to the N-terminal kinase domain (Figure 6). Binding of Rho to the RBD of ROCK induces the release of the kinase domain from the C-terminal domain, thus activating the kinase (Riento and Ridley, 2003) (Figure 6). 
Two ROCK isoforms have been identified, ROCK I and ROCK II (Nakagawa et al., 1996). The amino acid sequences of both ROCKs have $65 \%$ overall identity, and within the kinase domain the identity is $92 \%$, indicating ROCK's redundancy in substrate phosphorylation (Nakagawa et al., 1996). The isoforms differ in amino acid sequence within their PH domains, suggesting a possible source of specificity (Yoneda et al., 2005). ROCK I and ROCK II are ubiquitously expressed in tissues; however, the amount of protein expression varies among cell types. ROCK II seems to have a higher expression level in muscle cells and certain neuronal cells, which could indicate a predominant role in these types of cells (Leung et al., 1995; Nakagawa et al., 1996).

One of the hallmarks of both ROCK I and II activity is the phosphorylation of substrates that are linked to actin cytoskeleton contractility (Riento and Ridley, 2003). ROCK I and II negatively regulate myosin light chain phosphatase (MLCP) by phosphorylation at threonine 696 (Ito et al., 2004). Active MLCP negatively regulates myosin light chain activity by dephosphorylation (Kimura et al., 1996). In addition, ROCK I and ROCK II have been shown to directly phosphorylate serine 19 on myosin light chains, a main regulatory unit of non muscle myosin activity (Amano et al., 1996b). Pharmacological suppression of ROCK activity results in the loss of actin bundle formation, further supporting that the main function of ROCK is to regulate Rhodependent actomyosin contractility (Yoneda et al., 2005).

Another RhoA effector implicated in the regulation of cytoskeleton dynamics is the filamentous actin nucleating protein diaphanous Dia (Bishop and Hall, 2000). There are two isoforms of mammalian diaphanous, mDia 1 and mDia 2. Similarly to ROCK I and II, mDial and mDia 2 are activated by Rho binding to RBD (Wallar et al., 2007). The role of mDia is confined to the Arp2/3 independent polymerization of actin and formation of filopodia (Pellegrin and Mellor, 2005) and, it was recently shown, to 
mediate the formation of a subset of actin bundles (Hotulainen and Lappalainen, 2006) and stabilization of microtubules (Bartolini et al., 2008; Palazzo et al., 2001).

Rho GTPases are the main regulatory molecules of the actin cytoskeleton. Targeting Rho GTPase signaling to specific cellular compartments is achieved by selecting appropriate GEF and effector molecules that can activate the signals locally. In actively migrating cells, globally generated signals have to be directed to protrusions and trailing edges. In the following section I will discuss how globally generated signals coordinate cell migration.

\section{Signaling and cell migration}

Cell migration is initiated when cell surface receptors recognize a stimulus that leads to the formation of a predominant lamellipodium in the direction of migration (Ridley et al., 2003). The abundance of receptors for soluble factors as well as integrins on the cell surface allows the cell to bind various ligands and transform the ligand receptor interaction into intracellular signals (Ridley et al., 2003). Studies in neutrophils have revealed that receptors that are coupled to heterotrimeric $G$ proteins initiate intracellular signaling that drives polarized cell migration (Neptune and Bourne, 1997). Formyl-methionyl-leucine-phenylalanine (fMLP) binds to its receptor and activates the heterotrimeric protein $\mathrm{G}_{\mathrm{i}}$ (Neptune and Bourne, 1997). The $\beta \gamma$ subunits of $\mathrm{Gi}$ in neutrophils activate phosphotidylinositol kinase (PI3K) which in turn mediates localized production of phosphotidylinositol 3,4,5 phosphate (PIP3) and activation of Rac (Wang et al., 2002). Inhibition of heterotrimeric $\mathrm{G}_{\mathrm{i}}$ activation with Pertussis toxin (PTX) results in the failure of neutrophils to form lamellipodia and polarize (Srinivasan et al., 2003). Therefore $\mathrm{G}_{\mathrm{i}}$ activation is linked to lamellipodia protrusion by the local increase in PIP3 
production and Rac dependent actin polymerization. While Gi is distributed evenly across the cell surface of neutrophils, PIP3 is detected only in lamellipodia but not the trailing edge. The localized presence of PIP3 phosphatase in the trailing edge is responsible for turning off the PIP3 signal. The polarized distribution of PIP3 phosphatase ensures the polarity following G coupled receptor activation (Iijima et al., 2002).

During fMLP stimulation, another set of heterotrimeric G proteins is activated. The fMLP-mediated activation of G12/13 leads to the induction of Rho activity, followed by activation of ROCK, and the subsequent activation of actomyosin contraction and trailing edge retraction (Xu et al., 2003). The forced expression of a dominant negative form of $\mathrm{G} 12 / 13$, or the pharmacological inhibition of ROCK results in the failure of trailing edge retraction in neutrophils, indicating that the Rho pathway emanating from $\mathrm{G} 12 / 13$ is crucial to the control of trailing edge retraction during polarized migration $(\mathrm{Xu}$ et al., 2003). Rho A activation in the trailing edge of neutrophils is achieved by the coupling of PDZ-RhoGEF to actomyosin in the trailing edge (Wong et al., 2007). The experiments in neutrophils showed that signals initiated by heterotrimeric G proteins are spatially distributed by utilization of molecules that partition to the lamellipodium (PIP3) and the trailing edge (PDZ-RhoGEF).

In addition to receptors for soluble factors, the engagement of integrins with ECM has been shown to stimulate biochemical signals that support polarized protrusion formation (Ridley et al., 2003). Early studies indicated that cells plated on ECM components in the absence of growth factors extended protrusions in a similar fashion to adherent cells stimulated with growth factors (Schwartz, 2001). Subsequently it was shown that integrin -dependent protrusions correlated with tyrosine phosphorylation of the guanine nucleotide exchange factor Vav and activation of the small GTPases Rac and Cdc42 (del Pozo et al., 2003; Yron et al., 1999). Integrins activate protrusive signals by 
stimulating localized translocation of activated Rac to specific membrane domains found in the regions of active protrusions (del Pozo et al., 2004). Targeting GTP-Rac to plasma membrane domains depends on adhesion-mediated regulation of specific membrane domains called lipid rafts. In cells that are detached, lipid rafts containing Rac are fully internalized into endosomal compartments. Following cell attachment, lipid rafts along with GTP-Rac are transported via microtubles and actin to the plasma membrane to support active protrusions (Balasubramanian et al., 2007). In support of this model, studies show that active integrins, active Rac and lipid rafts are predominantly localized to protruding lamellipodia during cell migration (Del Pozo et al., 2002; Kraynov et al., 2000). It is likely that localization of active Rac to lamelipodia protrusions is mediated by de novo integrin-dependent activation of a Rac GEF that is localized to membrane domains that are absent from the trailing edge.

In summary, signal transduction pathways that drive polarized migration are spatially restricted to specific cellular locales. The protrusion of the lamellipodia is driven by localized activation of Rac and Arp2/3-dependent actin polymerization. Trailing edge retraction is achieved by activation of Rho and actomyosin contractility. Any deviation from the proper signal localization can change the migratory behavior of normal cells and lead to the development of disease. In the following paragraphs I will describe the plasticity of migratory behaviors that are developed during cancer.

\section{Cancer cell migration}

Cellular migration abnormalities have been attributed to pathological situations. Cancer metastasis is characterized by acquisition of new migratory proprieties that allow cells to disseminate to sites distant from the primary tumor. Tumor cells show great 
diversity in migratory behavior (Wolf and Friedl, 2006). For instance, tumors of connective tissue such as fibrosarcomas (Wolf et al., 2003) exhibit increased fibroblastlike (mesenchymal) migration. Mesenchymal tumor migration relies on integrindependent adhesion dynamics and actomyosin contractility (Itoh et al., 1999). Tumors of the kidney (Wood, 1958), mammary gland (Farina et al., 1998), lymphomas, leukemia (Verschueren et al., 1991) and small cell carcinoma of the lung and prostate (Rintoul and Sethi, 2001) migrate distinctly from connective tissue tumors. They tend to glide over the substrate without establishing classical lamelipodia and trailing edges (amoeboid like migration). These tumors express low levels of $\beta 1$ and $\beta 3$ integrins and have a low degree of adhesion to the extracellular matrix (Kraus et al., 2002; Rintoul and Sethi, 2001). This property allows them to readily detach and migrate from the site of the primary tumor. A third type of migration, so-called chain migration (Friedl and Wolf, 2003 ) is exhibited by non-neoplastic neuronal crest cells (Jacques et al., 1998), myoblasts (El Fahime et al., 2000), melanomas (Friedl et al., 1997), mammary carcinomas and ovarian cancers (Pitts et al., 1991; Sood et al., 2001). Chain migration is characterized by one cell following another in a strand like fashion. These cells tend to align and move along ECM fibrils preserving their top and bottom cell-cell junctional contacts. It is likely that this form of migration depends on adhesion dynamics and actomyosin contractility (Wolf and Friedl, 2006). Another example of cancer cell migration is collective migration and invasion (Friedl and Wolf, 2003). This type of behavior is similar to the migration of cell sheets during three layer embryogenesis or mammary gland morphogenesis (Davidson and Keller, 1999). The primary characteristic of collective sheet migration is the preservation of strong cell-cell adhesions that allow formation of cortical actomyosin assembly around the group of cells (Hegerfeldt et al., 2002). A subset of highly protrusive cells is on the margins of the sheet. Cells using this form of movement appear 
to use $\beta 1$ integrins and adhesion/actomyosin-dependent tension to move the sheet on the basement membrane (Friedl et al., 1995). In addition, these cells secrete metalloproteases to degrade the ECM and move the sheet through the basement membrane (Nabeshima et al., 2000) (Figure 7).

Migratory tumor cells show vast plasticity in switching between types of migration modes. It often happens that tumor cells compensate the loss of one particular migratory characteristic by acquiring another one (Wolf and Friedl, 2006). The well established example of this phenomena is epithelial-mesenchymal transition (EMT) (Thiery, 2002). Epithelial tumor progression and differentiation results in transition from a collective tumor migration into a single cell disseminated migration in which the cells move in fibroblast-like fashion and tend to lose cell-cell junctions (Moll et al., 1993. Another example of tumor cell plasticity is mesenchymal amoeboid transition (AMT) (Wolf et al., 2003). This transition is characterized by cell morphology changes (fibroblastic-like shape to round and ecliptoid-like shape), a decrease in integrin expression and proteolytic activities.

The plasticity of the migratory proprieties of tumor cells is dictated in large part by changes applied to the environment in which these cells live. For instance, pharmacological therapies involving inhibition of integrins and metalloproteases results in changes in tumor cell migration from collective and/or mesenchymal like migration to amoeboid like migration (Hegerfeldt et al., 2002; Zucker et al., 2000). On the other hand, reports show that tumor cells treated with cytostatic drugs and irradiation can revert from amoeboid-like migration to a collective migration pattern (Kraus et al., 2002). These examples clearly indicate that changes in migratory behavior of tumor cells represent forms of evasive mechanisms from existing cancer therapy. Therefore, it is crucial to decipher the molecular mechanisms that regulate different types of migration adopted by 
tumor cells during metastasis. One of the properties of highly metastatic tumors is hyperactivation of signaling pathways that regulate migration. In the following paragraphs, I will introduce selected protein kinases that have been implicated in the regulation of cell migration.

\section{Src protein tyrosine kinase.}

In 1909, Peyton Rous working with avian sarcoma tumors noticed that cell free extracts from tumors could initiate tumor outgrowth in healthy animals. Later it was discovered that tumor cell free extracts contained virus particles that were responsible for tumor outgrowth (Martin, 2004). Characterization of the RNA genome of one transforming isolate, designated Rous Sarcoma Virus (RSV) showed that a single viral gene was responsible for the transforming activity of the virus (designated $v$-Src). Using DNA renaturation techniques, it was shown that this transforming gene was a mutated version of a normal avian Src gene (designated c-Src)(Cordell et al., 1978). In the late seventies, researchers inoculating rabbits with RSV raised the first antibodies to the protein product of $v$-Src, Src protein (Brugge and Erikson, 1977; Brugge et al., 1978). Subsequently it was recognized that the Src protein was a unique protein kinase, capable of phosphorylating tyrosine residues (Sefton et al., 1980).

The comparison of the amino acid sequences of multiple member of the tyrosine kinase family revealed several highly conserved domains, designated SH1, SH2 and SH3 for Src homology domains 1, 2 and 3. In the case of c-Src, a myristolated N-terminal segment that is unique to Src is followed by the SH3 domains ( a domain that binds to proline-rich sequences), the $\mathrm{SH} 2$ domain (a recognition domain for phosphotyrosine containing sequence motifs), a linker region, the SH1 domain (the highly conserved 
kinase domain) and a short $\mathrm{C}$-terminal tail containing negative regulatory tyrosine residue 527 (Boggon and Eck, 2004). Crystallographic analysis of inactive c-Src revealed that the SH3 and SH2 domains turn inward over the kinase domain and lock the kinase domain in an inactive confirmation (Schindler et al., 1999) by virture of an interaction between the c-terminal phoshotyrosine and the SH2 domain. Activation of c-Src is achieved by the release of the $\mathrm{SH} 3$ and $\mathrm{SH} 2$ domains from the kinase domain and by removal of the $\mathrm{C}$ terminal phosphotyrosine. This event is initiated by protein phosphatase-dependent dephosphorylation of the negative regulatory phosphorylation on tyrosine 527 or high affinity ligand binding to the SH3 domain and release of intramolecular interactions within c-Src (Arias-Salgado et al., 2003). Following opening of the molecule, c-Src phosphorylates itself on tyrosine 416 within the kinase domain and achieves the complete activation state (Parsons and Parsons, 2004).

Activation of c-Src has been shown to take place downstream of substrate adhesion receptors (Shattil and Brugge, 1991). There are a series of experiments demonstrating that c-Src participates in adhesion dependent signaling (Playford and Schaller, 2004). First, Src has been found to interact directly with the $\beta 3$ integrin subunit (Arias-Salgado et al., 2003) and adhesion components like FAK, paxillin and the scaffold protein p130 Cas (Parsons et al., 1994). Second, various forms of activated Src have been shown to localize to adhesion structures in fibroblastic cells (Yeo et al., 2006). Third, in Src-transformed cells, a adhesion-associated proteins showed increased tyrosine phosphorylation (Schaller et al., 1993). One of the proteins that showed increased phosphorylation in cells transformed with activated Src was FAK. In the following paragraphs I will describe proprieties of FAK as a main component of adhesion signaling. 


\section{FAK structure and regulation.}

FAK is a non-receptor tyrosine kinase of molecular weight $124 \mathrm{kDa}$ (Guan et al., 1991; Hanks et al., 1992; Schaller et al., 1992). FAK is closely related to the protein tyrosine kinase PYK2. FAK and PYK2 share different degrees of sequence similarity within specific domains, with the highest similarity within the the kinase domains (60\%). The amino terminal domain of FAK is termed the FERM domain (erythrocyte band four.1-ezrin-radixin-moesin), because of its sequence similarity to the domains found in JAK family kinases as well as, ezrin, radixin and moesin (Girault et al., 1999). FERM domains have been shown to interact directly with transmembrane receptors as well as phosphoinositides (Di Paolo and De Camilli, 2006; Garcia-Alvarez et al., 2003). The FERM domain of FAK is a three lobed protein-interaction domain (Lietha et al., 2007). Previous experiments demonstrated that this domain interacts with peptides derived from the cytoplasmic tails of $\beta 1$ integrins (Schaller et al., 1995) and co-precipitates with the receptors for EGF and PDGF (Sieg et al., 2000). Using lipid protein overlay assays, it has been recently shown that the FERM domain of FAK interacts with phosphoinositide 4,5 phosphate (PIP2) (Cai et al., 2007). The FERM domain is connected to the central kinase domain via a 40 residue linker region containing a proline-rich domain that is positioned adjacent to tyrosine 397 , which is the major autophosphorylation site within FAK. The Cterminal region of FAK is composed of 150 amino acids called the focal adhesion targeting domain (FAT). Located between the catalytic and FAT domain are two prolinerich sequences that mediate interactions with SH3-domain containing proteins (Playford and Schaller, 2004).

The crystal structure of autoinhibited FAK reveals that the FERM domain interacts with the kinase domain through the F-2 lobe of FERM and the C-lobe of the 
kinase domain (Lietha et al., 2007). This intramolecular interaction creates a structure where the FERM domain blocks access to the active site cleft (where ATP binds) and to the kinase activation loop (required for transfer of the phosphate). Therefore, the proposed mode of FAK activation is release of the FERM domain from the kinase domain (Lietha et al., 2007) (Parsons et al., 2008) ( Figure 8). A likely hypothesis for FAK activation is that release of the FERM domain from the kinase domain is mediated by a high affinity ligand for the FERM domain.

FAK has been shown to be activated in response to integrins and soluble factor receptors. The mechanism of FAK activation by soluble factor receptors is poorly understood but it involves participation of Rho GTPases (Seufferlein and Rozengurt, 1994). On the other hand, integrin-dependent activation of FAK is well studied. Adhesion to a variety of ECM components induces tyrosine phosphorylation of FAK (Guan et al., 1991) predominantly on tyrosine 397. Current evidence is consistent with a model whereby the clustering of integrins on the cell surface via engagement with the ECM leads to the aggregation of the cytoplasmic tails of $\beta$-integrins $(\beta 1, \beta 3$ and $\beta 5)$. This directly or indirectly leads to the recruitment of FAK, FAK activation (possibly through binding to the FERM domain of FAK, thus, relieving negative regulation) (Toutant et al., 2002) and outophosphorylation of tyrosine 397. Tyrosine phosphorylation of residue 397 creates a high affinity binding site for the SH2 domain of Src family kinases (Schaller et al., 1994) (Figure 8). The binding of Src to FAK leads to the activation of Src and downstream phosphorylation of adhesion substrates including paxillin and p130 Cas. 


\section{FAK localization in cells.}

Immunostaining of chicken embryo fibroblasts with FAK-specific antibodies revealed that FAK was concentrated in the cell -ECM adhesion sites (Schaller et al., 1992), (Hanks et al., 1992). In addition, over-expression of FAK linked to GFP resulted in the localization of a large fraction of GFP-FAK to adhesion structures. However, FAK does not localize to adhesions that develop into $\alpha 5$ integrin-containing fibrils (fibrillar adhesions) (Cukierman et al., 2001). Therefore, FAK localization into fibrillar adhesions is subjected to negative regulation most likely involving the usage of $\beta$ integrin subunit subsets. In fibroblasts grown in a three-dimensional environment, FAK is localized to adhesions but fails to be tyrosine phosphorylated (Cukierman et al., 2001), indicating low integrin clustering at these sites (Cukierman et al., 2001). In microphages, FAK localizes with vinculin rich adhesions (Owen et al., 2007).

\section{FAK signaling.}

Phosphorylation of FAK in response to cell adhesion to the ECM leads to the formation of phosphotyrosine docking sites for several classes of signaling molecules. In addition to phosphotyrosine-mediated binding of proteins to FAK, the proline-rich domains found within the C-terminal region of FAK facilitate interaction with several other signaling proteins (Parsons, 2003). The most critical event in initiation of FAK downstream signaling is the clustering of integrins and integrin-dependent FAK tyrosine phosphorylation at position 397 (Schwartz, 2001). Phosphorylation of tyrosine 397 is

crucial for the recruitment of Src to FAK and subsequent Src dependent tyrosine phosphorylation of FAK and FAK/Src-dependent phosphorylation of additional 
substrates (Schaller et al., 1994; Schaller et al., 1995; Schaller and Parsons, 1995). Phosphorylation of Tyr397 also appears to be important for the recruitment of other SH2 domain containing proteins, including phosphoinositide 3 kinase (Akagi et al., 2002; Chen et al., 1996) and the adaptor growth factor receptor bound protein - 7 (Grb-7) (Han and Guan, 1999). The C-terminal domain of FAK has multiple protein-protein interaction sites. There are two proline-rich domains upstream of the FAT domain within the Cterminal region of FAK. The first proline-rich domain (Site I) provides the major binding sequence recognized by the SH3 domain of p130 Cas, a multifunctional adaptor protein (Harte et al., 1996). The second proline-rich domain (Site II) binds the SH3 domains of two regulators of small GTPases: GTPase regulator associated with FAK (GRAF) (Hildebrand et al., 1996) and a guanine activating protein for Arf1 and Arf6 GTPAses ( ASAP1) (Liu et al., 2002). The FAT domain of FAK has been shown to bind paxillin, talin (Chen et al., 1995) and the Rho guanine exchange factor (p190 RhoGEF) (Zhai et al., 2003). In addition, tyrosine 925 within the FAT domain has been shown to be a binding site for growth factor receptor bound protein (Grb2) (Schlaepfer et al., 1998) which in many systems has been shown to initiate extracellular regulated kinase signaling (ERK) (Kolch, 2005).

One of the ways to activate the ERK pathway during integrin engagement is linking FAK to the canonical Ras pathway. The recruitment of Grb2 to FAK initiates the recruitment of the Ras GTPase exchange factor Son of Sevenless (SOS), which in turn activates Ras by catalyzing the exchange of GDP for GTP. The plasma membrane associated GTP-loaded Ras recruits its effector Ras activated factor (Raf), a serine threonine kinase. Membrane-assosicated Raf is activated by upstream kinases including Src (Hood et al., 2003) and PAK (King et al., 1998). Activated Raf phosphorylates and 
activates MAP kinase and ERK kinase (Bourett et al.), which leads to ERK activation (Renshaw et al., 1996) (Schlaepfer et al., 1999).

Activation of Raf can be also independent of the Ras pathway. Protein kinase C alpha $(\mathrm{PKC} \alpha)$ can directly phosphorylate Raf and induce ERK activation distinct from canonical Ras pathway (Kolch et al., 1993). MEK and ERK are thought to be core components of the ERK pathway and are presented together by scaffold molecules for upstream activation by either integrins or growth factor receptors (Kolch, 2005).

The interaction of FAK with multiple binding partners opens many possibilities for integrin-dependent signal transduction pathways. The interaction of FAK with small GTPase regulating proteins and upstream ERK activators raises the possibility that FAK co-ordinates biological processes through the regulation of actin cytoskeleton.

\section{Genetic analysis of FAK function.}

The first insights into biological functions of FAK came from studies analyzing embryos from mice in which the FAK gene had been disrupted (Ilic et al., 1995). Homozygous mutant mouse embryos at day 8.5 exhibited poorly developed mesoderm with no notochord or somites. Whereas the heart and vasculature initially developed normally, their growth was subsequently retarded. The overall phenotype was lethal and similar to that observed in mice lacking fibronectin (George et al., 1993) or $\beta 1$ integrin, suggesting that FAK contributes to fibronectin-integrin interactions supporting development. Isolation of fibroblast-like cells from mutant embryos revealed that FAK mutant cells were circular and formed larger adhesions and migrated slower when compared to control cells (Ilic et al., 1995). These studies concluded that FAK was essential for mesoderm development and that the developmental abnormalities observed 
were a consequence of impaired cellular migration. However, due to the early developmental lethality of FAK mutant embryos, it was impossible to assess the role of FAK in different tissues.

The generation of mice with Cre-mediated targeted deletion of the FAK gene in differentiated tissues (Beggs et al., 2003) has allowed a more thorough analysis of the function of FAK in differentiated cells. Targeted deletion of FAK in the brain shows a role for FAK in the organization of the forebrain. Cortical lamination of basement membrane beneath the forebrain in tissues lacking FAK was disorganized. Disruption of FAK expression in the developing dorsal forebrain resulted in neuronal ectopias, manifested by misplacement of cells within the marginal zone of the forebrain. Cells often migrated into inappropriate compartments. Finally neurons from FAK-deleted brains exhibited increased dendrite branching and loss of dendrite orientation. Overall these studies showed that FAK is crucial for brain basal lamina organization and proper regulation of directional neuronal migration within the forebrain (Beggs et al., 2003).

The conditional deletion of FAK in osteoblasts showed that FAK-deficient bone cells were delayed in wound healing (Kim et al., 2007). In addition, the deposition of basal lamina and ECM mineralization was disrupted in FAK mutant osteoblasts. Overall, this study concluded that FAK is necessary for the organization of the ECM and proper cellular migration during bone injury.

Studies in mice where FAK was deleted in endothelial cells revealed that formation of vasculature is FAK dependent (Braren et al., 2006). Mice with targeted deletion of FAK in the endothelium exhibited irregular formation of vasculature resulting in hemorrhage and lethality. Endothelial cells isolated from FAK mutant mice showed increases in cell retraction, irregular migration and cell death during vascular formation. In another study, targeted deletion of FAK in cardiomyocytes revealed that FAK is 
necessary for normal heart function (Hakim et al., 2007; Peng et al., 2006). FAK mutant mice died shortly after birth and the phenotype was traced to subaortic ventricular septal defects and abnormal alignment of the outflow track of the heart. In addition, the myocardium of FAK mutant mice exhibited disorganized myofibrils. Cardiomyocytes isolated from FAK mutant mice exhibited abnormal migration to chemotactic stimuli.

In a different study, the loss of FAK in the epidermis resulted in misregulation of the hair cycle, sebaceous gland hypoplasia and epidermal thinning (Essayem et al., 2006). Primary keratinocytes isolated from the epidermis of FAK mutant mice did not proliferate in culture and died within 48 hours, indicating that FAK is required for keratinocyte growth. However, in another study, FAK deficient keratinocytes were propagated on fibroblast feeder layers, with no defects in proliferation. In this study the major observed defect was abnormal keratinocyte migration (Schober et al., 2007).

In summary, studies using targeted disruption of the FAK gene have clearly shown that FAK deletion resulted in primary deficits in the organization of basal lamina in specific tissues. In addition, in all these studies, cellular migration was disturbed cells migrated but not efficiently, often lacking direction. These results point to the role of FAK signaling in focal adhesion/cytoskeleton organization and dynamics. Proper regulation of adhesions and the cytoskeleton have been shown to influence ECM organization in tissue culture cells (Ilic et al., 2004; Yoneda et al., 2007). Regulation of focal adhesion dynamics in tissue culture cells has been previously shown to depend on FAK and its activity (Webb et al., 2004). In particular, FAK signaling to Rho and ERK was implicated as a major pathway regulating adhesion dynamics (Lim et al., 2008; Palazzo et al., 2004; Ren et al., 2000) (Webb et al., 2004). Interestingly, FAK expression and tyrosine phosphorylation are often correlated with the metastatic potential of many 
tumor cells. In the following section, I will describe the current evidence that FAK initiated pathways in cancer cells could be a potential target for therapeutic interventions.

\section{FAK and cancer progression.}

FAK was initially identified as a major tyrosine phosphorylated substrate in vSrc transformed chicken embryo fibroblasts (Schaller et al., 1992). It was subsequently demonstrated that Src directly interacted with FAK and phosphorylated FAK in vitro (Schaller et al., 1994). Later, it was shown that the human fak gene localized to chromosome 8 and was amplified in cell lines derived from metastatic tumors of head, neck and lung cancer (Agochiya et al., 1999). Moreover, FAK mRNA was increased in 49 neoplastic human tissue samples, including adenomatous tissues, invasive tumors and metastatic tumors of different origins (Weiner et al., 1993). In another study, the authors showed that levels of FAK expression were increased by $100 \%$ in colon cancer and $88 \%$ in breast cancer (Owens et al., 1995). In a variety of tumors, the overexpression of FAK was correlated with advanced disease and poor survival prognosis (Cance et al., 2000). FAK overexpression in ovarian cancer patients was linked to metastasis to the lymph nodes and distant organs (Sood et al., 2004). These findings suggested the possibility that FAK protein levels may be a prognostic marker of cancer disease progression (McLean et al., 2005).

The mechanism(s) that underlies FAK overexpression in tumors is not fully understood. Chromosomal polyploidy has been implicated in fak gene amplification in metastatic head, neck and lung cancer cell lines (Agochiya et al., 1999). Recent studies of the fak gene promoter indicate that FAK expression is regulated by the level of p53 tumor suppressor protein (Golubovskaya et al., 2007). Using chromatin immunoprecipitation 
(Chipperfield and Pyle) assays it was demonstrated that p53 directly bound to the promoter region of the fak gene and that mutation of sites in p53 that mediate interactions with the fak promoter abolished the interaction. In addition, ectopic expression of p53 in cancer cells decreased expression of FAK. These studies indicate that transcriptional silencing of fak could involve regulation of p53 binding to the FAK promoter.

The FAK - Src signaling complex acts to recruit and phosphorylate a number of signaling proteins that are involved in the motile and invasive phenotype (Hsia et al., 2003; Schlaepfer et al., 1999). Increased tyrosine 397 phosphorylation of FAK has been reported to be associated with different tumors (Gabarra-Niecko et al., 2003). For instance, in ovarian and breast tissue, increased tyrosine phosphorylation of FAK was found in invasive tumors but not in benign tumors or normal epithelium (Sood et al., 2004).

Whereas there is little doubt that biochemical properties of FAK are changed in tumor tissues and cells, most of the evidence connecting FAK to tumorogenesis has been correlative. Today there are no data indicating that FAK mediates tumor formation or tumor progression. Ectopic over-expression of FAK in normal cells does not promote uncontrolled growth and transformation, a phenotype that is characteristic of Src overexpression. Nevertheless, the observations that FAK is overexpressed in multiple cancers and that it's expression pattern correlates with poor clinical outcome indicate that FAK might be a useful therapeutic target. 


\section{Figures}

\section{Figure 1: Schematic representation of selected ECM proteins.}

(A) Collagen trimer is composed of two $\alpha 1$ and one $\alpha 2$ subunits. Collagen subunits interact via $\alpha$ helical collagenous regions. Non collagenous regions are positioned at the C-terminus of each subunit. (B) Laminin heterotrimer contains one $\alpha$, one $\beta$, and one $\gamma$ subunit. Alpha subunit contains five $\mathrm{G}$ domains positioned at the $\mathrm{C}$ terminus. $\mathrm{N}$ domains are present at the $\mathrm{N}$ terminus of each subunit. (C) Fibronectin contains modules of type I,

II and type III repeats. Alternative (AS) splicing of type III repeat exons can generate various fibronectin molecules (yellow). 
Figure 1

(A)

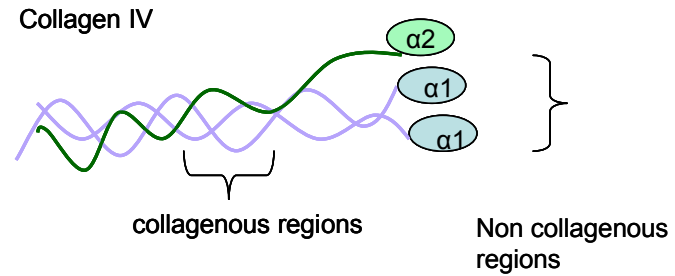

(C)

Fibronectin

Type II

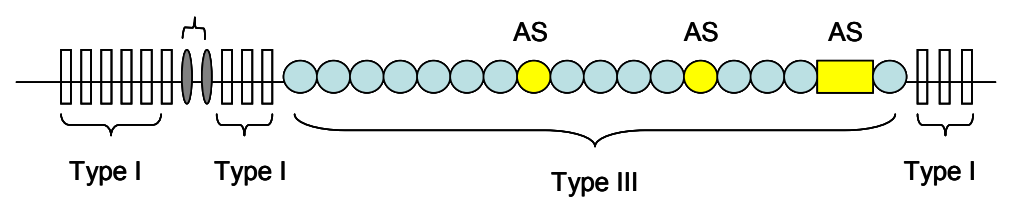

(B) Laminin

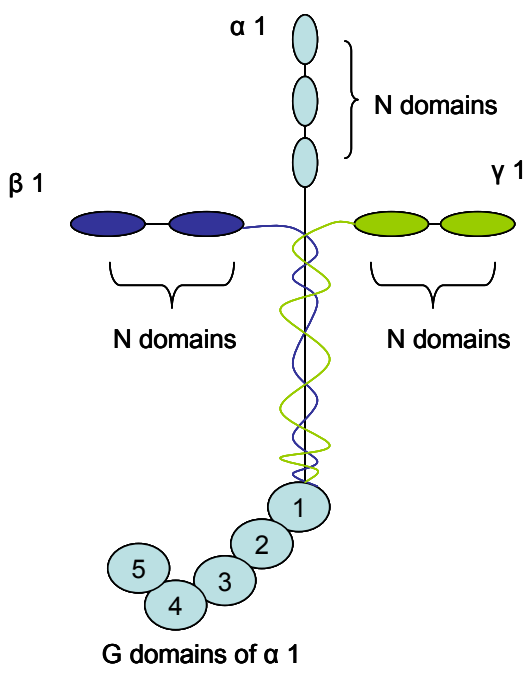


Figure 2. Schematic representation of $\alpha$ and $\beta$ subunits of integrins.

Integrin, $\alpha$ and $\beta$ subunits are transmembrane glycoproteins with short cytoplasmic tails.

The $\mathrm{N}$ terminus of the $\alpha$ subunit contains the amino acid sequence (Asp- $\mathrm{x}-\mathrm{Asp}-\mathrm{x}$-AspGly-x-x-Asp) that supports divalent cation binding and is necessary for proper integrin function (I domain region). Common to all $\beta$ subunits is a four fold repeat of a cystine rich segment within the $\mathrm{N}$ terminal portion of the subunit. These repeats are thought to be important in ligand binding. 
Figure 2

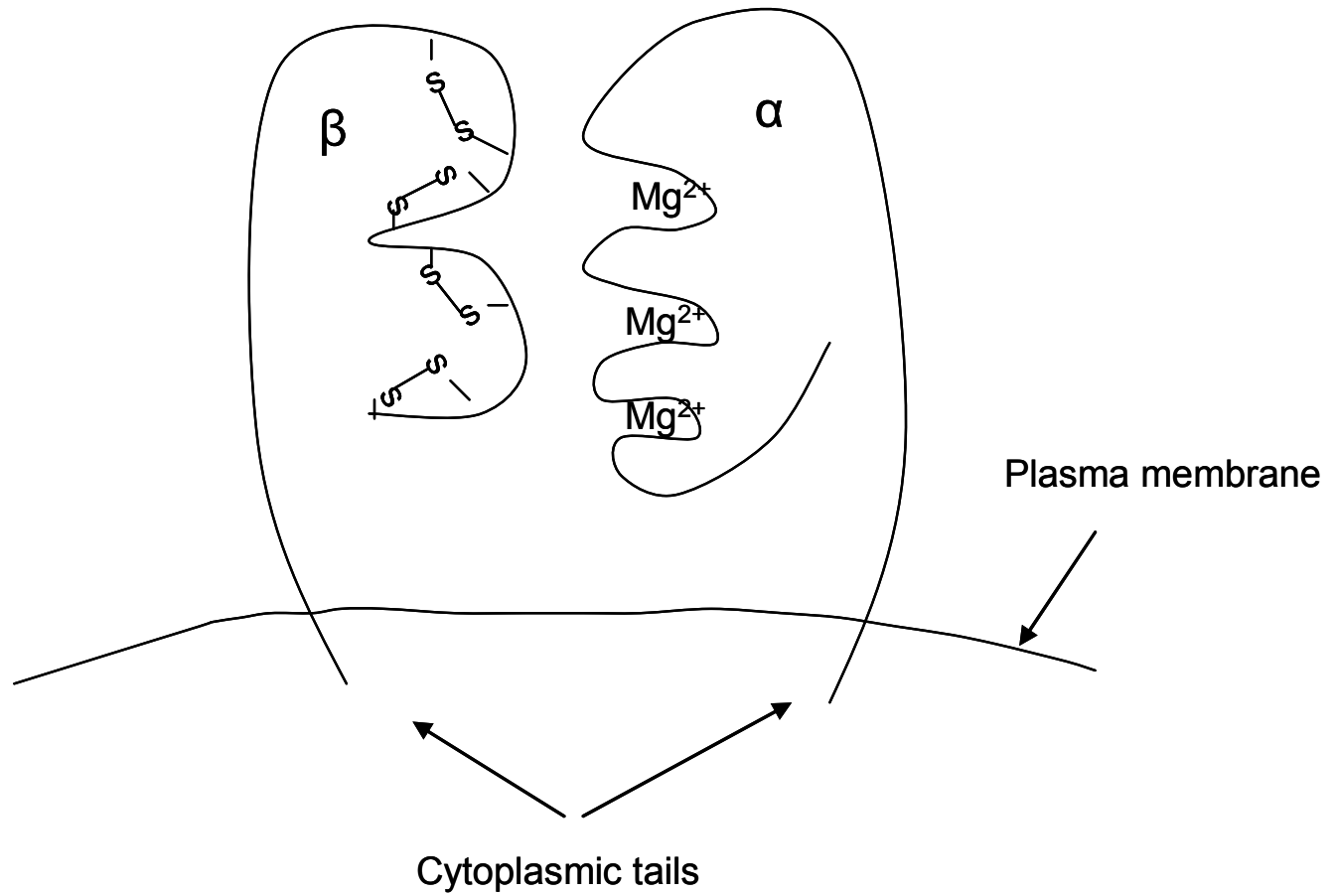


Figure 3. Schematic representation of myosin molecule.

Every myosin II molecule is a hexamer composed of Myosin Heavy Chain dimers (MHC) and two pairs of Myosin Light Chains (MLC). Myosin binds actin in a reversible manner and hydrolyzes ATP. The N-terminus of MHC is a globular head that contains ATPase activity and binds actin. The C-terminus of $\mathrm{MHC}$ is involved in myosin filament formation. 
Figure 3

Myosin

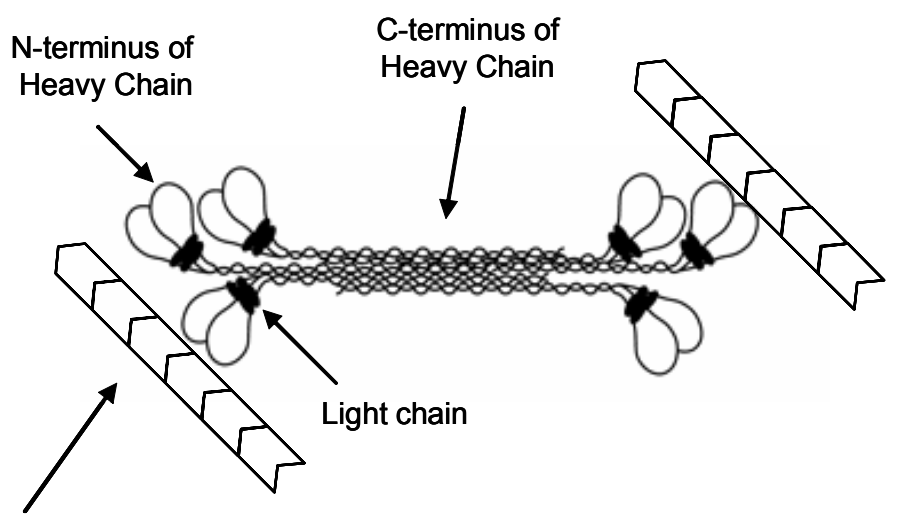

Actin filament 
Figure 4. Schematic representation of the cytoskeletal components during cell migration.

(A) Cell migration is characterized by the formation of predominant a broad, protruding, leading edge and a narrow, trailing edge. Leading-edge protrusions are driven by Arp2/3dependent actin polymerization and bending of the polymerized actin network. Protrusion is coupled to the formation of integrin-ECM adhesions, that can either disassemble or mature. Disassembly of some of the adhesions is facilitated by targeting of stable microtubules. Some of the formed adhesions will mature due to interaction with the actomyosin network. In the trailing-edge adhesions are elongated and tend to move inward during trailing edge retraction. (B) Adhesion movement (treadmilling) is characterized by simultaneous disassembly of one part of adhesion and assembly of another part of adhesion along the actomyosin cytoskeleton. 
Figure 4

(A)

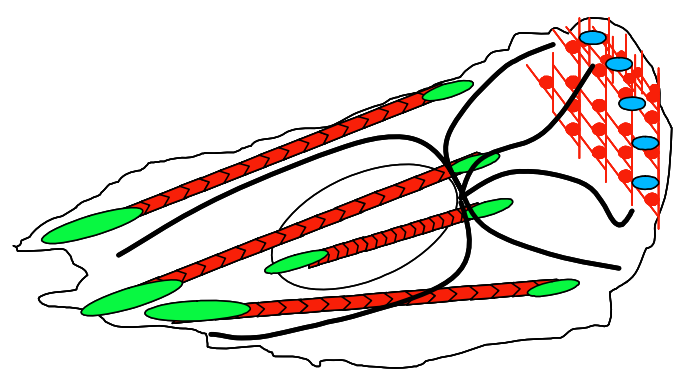

Arp2/3 and actin at the leading edge

- nascent adhesions

$\bigodot$ mature adhesions

actomyosin bundles

$\longrightarrow$ microtubules

(B)

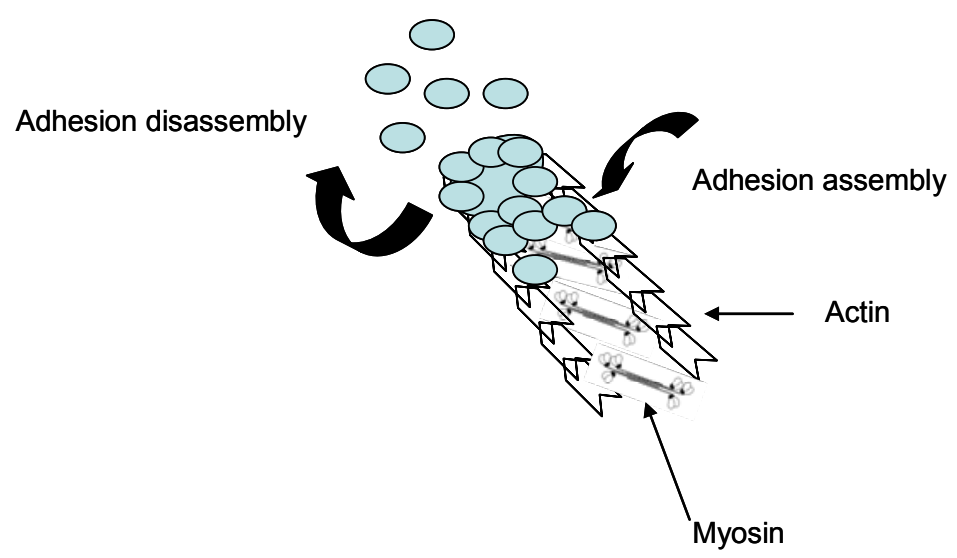


Figure 5. Domain composition of PDZ-RhoGEF and p115-RhoGEF.

PDZ-RhoGEF and p115 RhoGEF belong to the RGS domain-containing GEFs that link heterotrimeric G protein signaling with Rho activation. p115 RhoGEF and PDZ-RhoGEF via the regulator of G-protein signaling homology sequence (RGS) bind to Ga 12/13 and increase its nucleotide catalytic activity (Chikumi et al., 2004). Ga12/13 binding to the RGS domain of these GEFs promotes GEFs PH/DH domain binding to Rho, and Rho activation. In contrast to p115-RhoGEF, PDZ-Rho GEF has PDZ and actin binding (Horne et al.) domains. 
Figure 5.

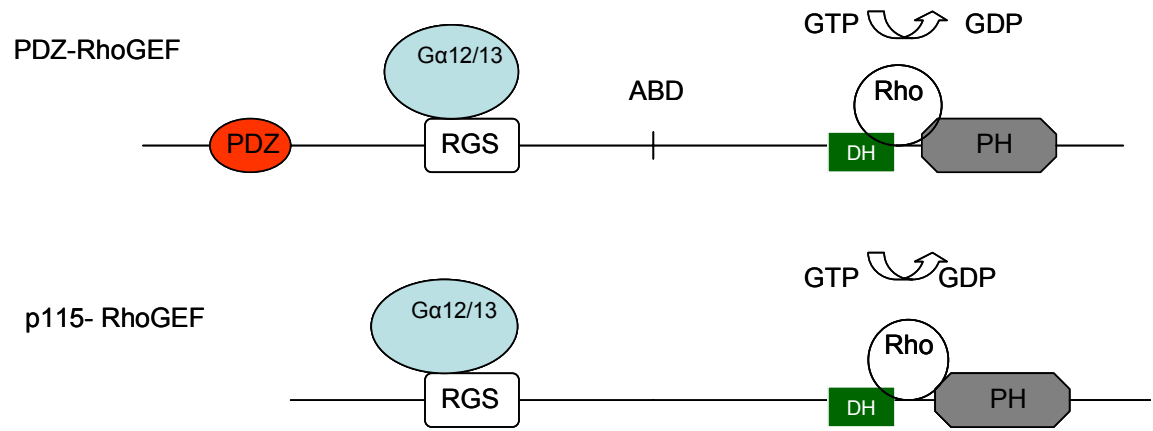


Figure 6. Schematic representation of Rho-induced activation of ROCK.

ROCK belongs to the family of serine threonine kinases. The ROCK contains a Nterminal kinase domain, Rho binding domain $(\mathrm{RBD}$,) pleckstrin homology domain $(\mathrm{PH})$ and cystine rich region (CRD). In the inactive state, the C-terminal RBD, $\mathrm{PH}$ and $\mathrm{CRD}$ domains of ROCK are folded over and bound to the N-terminal kinase domain. Binding of Rho to the RBD of ROCK induces release of the kinase domain from the C-terminal interaction, thus activating the kinase. 
Figure 6

Activation of ROCK

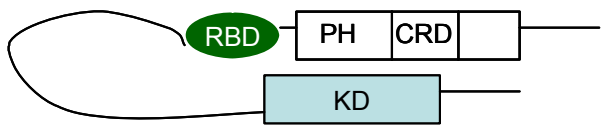

Inactive ROCK

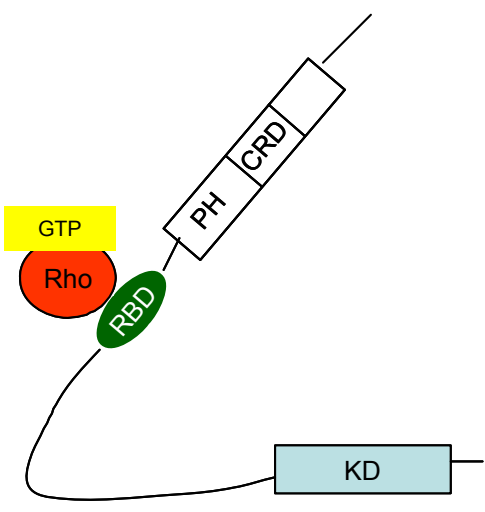

Active ROCK 
Figure 7. Schematic representation of migratory proprieties that cancer cells can adopt.

In amoeboid-like migration cells tend to glide over the substrate without establishing classical lamelipodia and trailing edges.

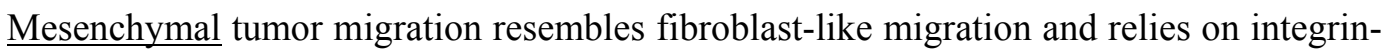
dependent adhesion dynamics and actomyosin contractility.

Chain migration is characterized by one cell following another in a strand-like fashion. These cells tend to align and move along ECM fibrils preserving their top and bottom cell-cell junctional contacts. It is likely that this form of migration depends on adhesion dynamics and actomyosin contractility.

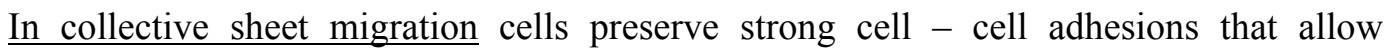
formation of cortical actomyosin assembly around the group of cells. On the margins of the sheet is a subset of highly protrusive cells. Cells using this form of movement appear to use $\beta 1$ integrins and adhesion/actomyosin-dependent tension to move the sheet on the basal membrane. In addition these cells secrete metalloproteases to degrade matrix and move the sheet and invade through the basal membrane 
Figure 7

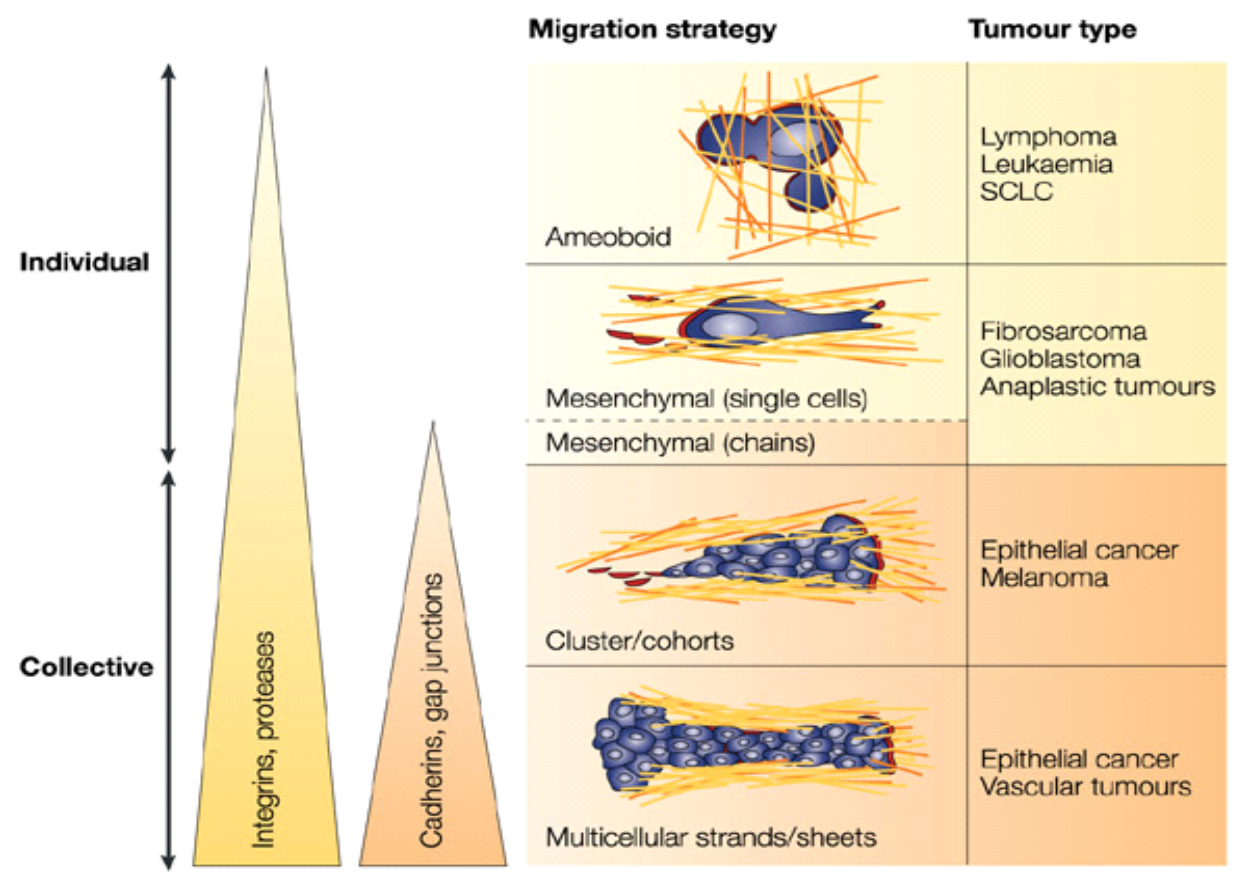

Adapted from :Peter Friedl \& Katarina Wolf Nature Review Cancer 2003 


\section{Figure 8. Integrin-dependent activation of FAK}

Engagement of integrins with ECM proteins results in recruitment of FAK to integrin $\beta 1$ subunit cytoplasmic domains and release of the FERM domain from the kinase domain of FAK. FAK in its open confirmation is able to transphosphorylate itself at tyrosine 397. Phosphorylated tyrosine 397 provides a docking site for Src, which also interacts with the proline rich region of the FAK positioned upstream of tyrosine 397, initiating FAK/Src complex formation. 
Figure 8
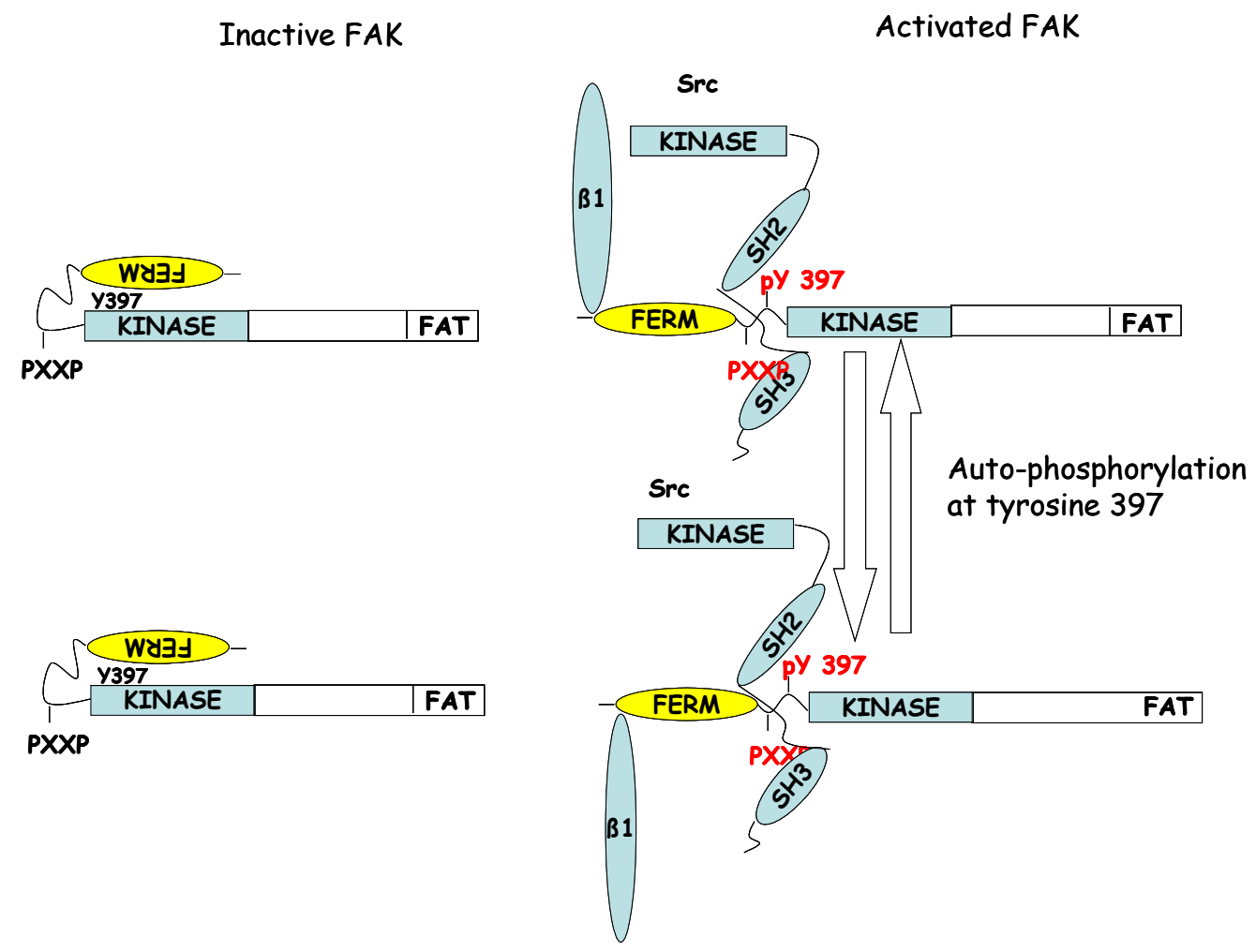


\section{Dissertation focus.}

Cell migration is initiated by the formation of a leading edge lamellipodium and a distinct trailing edge. Lamellipodial substrate adhesions constantly form and disassemble whereas trailing edge adhesions tend to move inward and break during retraction. It is likely that signals regulating adhesion dynamics in the lamellipodia differ from the regulation of adhesions found in the trailing edge. In Chapter 2 and Chapter 3 of this thesis, I argue that the spatial coupling of distinct signaling pathways contributes to differential adhesion behavior found in migrating fibroblasts.

In the first part of this work I show that regulation of adhesions found within the trailing edge and concomitant trailing edge retraction in fibroblasts is regulated by growth factor-dependent regulation of FAK signaling to Rho. We chose a model in which adhesion dynamics and trailing edge retraction are promoted by activation of LPA receptors that induce Rho activation. Using this model, we discovered that FAK forms a complex with PDZ-RhoGEF. PDZ- RhoGEF localized to FAK-containing adhesions, and attenuation of either FAK or PDZ-RhoGEF resulted in the failure of LPA to induce adhesion dynamics and trailing edge retraction. Furthermore, we found that LPAmediated adhesion dynamics and trailing edge retraction was also dependent on ROCK II signaling.

In the second part of this work which was performed in collaboration with Tomas Vomastek, I show that regulation of adhesions found in the lamellipodia depends on integrin/FAK-dependent RACK 1 targeting of the ERK pathway to de novo formed adhesions. We found that integrin-linked scaffold protein, RACK-1, channels ERK to adhesion sites for upstream activation and promotes adhesion disassembly and cell 
migration. Attenuation of either RACK-1 or FAK interferes with active ERK localization to adhesions and inhibits adhesion disassembly in the lamellipodia.

In this dissertation I demonstrate that FAK signaling to Rho and the ERK pathway is critical for the regulation of different aspects of adhesion dynamics. Our work emphasizes that regulation of adhesion dynamics within distinct parts of a migrating cells depends on differential focusing of signals initiated by integrins and soluble factor receptors.

Plasticity of tumor cell migration is a result of aberrant regulation of the signals that control different migratory steps. Therefore, our work helps to understand the principles of cell migration which, when uncontrolled, could lead to tumor metastasis. 


\section{CHAPTER 2}

\section{FAK couples Rho Signaling to Adhesion Movement and Trailing} Edge Retraction in Fibroblats

\section{Introduction}

Fibroblast migration is a highly regulated process (Ridley et al., 2003). Protrusion of the leading edge and the establishment of new peripheral adhesions coupled with the maintenance of stable attachments at the cell rear create the typical asymmetrical profile of a migrating cell with a broad lamellipodia (front) and narrow long trailing edge (rear) (Regen and Horwitz, 1992). As the lamellipodium advances and trailing edge stretches the cell becomes elongated. Inward movement and subsequent release of adhesions at the rear lead to trailing edge retraction, cell body advance, and the cell becomes more rounded (symmetrical), and the cycle is repeated (Rid et al., 2005; Smilenov et al., 1999; Webb et al., 2005). Fibroblast movement requires spatial regulation of cell attachment to extra-cellular matrix (ECM). Adhesions are formed at sites of lamellipodial protrusion. Once formed, these adhesions must be strong enough to generate traction forces necessary to support translocation of the cell body. On the other hand, the trailing edge of the cells must be able to detach from the substrate to permit cell translocation (Regen and Horwitz, 1992). Detachment of the trailing edge requires continued myosin-driven cytoskeletal tension that results in movement and ultimate release (disassociation/degradation) of adhesions within the trailing edge (Palecek et al., 1998; Palecek et al., 1996; Rid et al., 2005; Smilenov et al., 1999). 
LPA stimulation of several cell types induces tyrosine phosphorylation and activation of Focal Adhesion Kinase (FAK) (Bian et al., 2006; Luttrell et al., 1999; Seufferlein and Rozengurt, 1994). FAK is a non-receptor tyrosine kinase that is associated with adhesions (Hildebrand et al., 1993; Schaller et al., 1992) and sites of close contact between the cell and the extracellular matrix. FAK is activated in response to integrin engagement and its phosphorylation leads to the recruitment and activation of Src and the induction of several downstream signaling pathways (Parsons, 2003; Parsons et al., 1994). In addition FAK serves as a scaffold for the recruitment of the linker proteins, Cas (Burnham et al., 1996) and two GTPase activating proteins, ASAP (Liu et al., 2002) and GRAF (Hildebrand et al., 1996). Thus, FAK functions to recruit proteins that contribute to the regulation of adhesion signaling and promotes the dynamic remodeling of adhesions by triggering adhesion disassembly through a Src- and MEKdependent cascade (Webb et al., 2004). Cells deficient for FAK exhibit defects in adhesion disassembly (Webb et al., 2004) and Rho regulation upon integrin (Ren et al., 2000) or LPA receptor stimulation (Palazzo et al., 2004)

In the present study, we investigate the role of LPA, FAK and PDZ-RhoGEF in the regulation of adhesion movement, trailing edge retraction and cell shape change. We observed that maintaining fibroblasts in the absence of serum results in stabilization of adhesions within the rear of the cell and significantly reduces trailing edge retraction resulting in an elongated cell phenotype. LPA but not PDGF was sufficient to restore trailing edge retraction and increase inward movement of adhesions, and to restore cell shape. Thus, the response of serum-deprived cells to LPA parallels events leading to tail retraction in a migrating cell. Using this paradigm, we provide evidence that LPA induces dynamic movement of adhesions present in the trailing edge and this process requires FAK, PDZ-RhoGEF and Rho/ROCK II function. This study provides evidence for the 
spatial activation of an LPA receptor/FAK/ PDZ-Rho GEF signaling complex and suggests that modulation of adhesion movement within the trailing edges of fibroblasts is linked to Rho/ROCK II-dependent retraction. 


\section{Materials and Methods}

\section{Cell Culture, plasmids and siRNAs.}

Rat2 and HEK293T cells were maintained in DMEM plus $10 \%$ fetal calf serum (GibcoBRL) at $37^{\circ} \mathrm{C}$ in $5 \% \mathrm{CO}_{2}$. NIH3T3 cells were maintained in DMEM plus $10 \%$ calf serum (GibcoBRL), at $37^{\circ} \mathrm{C}$ in $5 \% \mathrm{CO}_{2}$. For the FAK and PDZ-RhoGEF knockdown experiments, cells were transfected with siRNA oligonucleotides specific for rat FAK, GTRAP48 (PDZ-RhoGEF, Dharmacon pool) or with control siRNA oligonucleotides using calcium phosphate as previously described (Tilghman et al., 2005). FAK, ROCKI and ROCK II shRNA plasmids were obtained from Sigma (USA). For transient expression of myc-PDZ-RhoGEF, myc-PDZ-RhoGEF 1-585, myc-p115RhoGEF and GFP-FAK, Rat2 or HEK293T cells were plated in the presence of $10 \%$ serum in $100 \mathrm{~mm}$

${ }^{2}$ plastic culture dishes at densities $4 \times 10^{5}$ and $1.5 \times 10^{6}$ cells per dish respectively. The next day cells were transfected using Polyfect reagent (Qiagen) with $0.25 \mu \mathrm{g}$ or $0.5 \mu \mathrm{g}$ of the designated plasmid. Twenty four hours later cells were processed for experiments.

The Flp-In System (Invitrogen) was used to create stable cell lines expressing GFPtagged or RFP-tagged proteins. Briefly, NIH3T3-LacZeo or RAT2-LacZeo cell lines with a flp recombinase target (FRT) site integrated in the genome were created. cDNAs encoding either Paxillin-GFP, GFP-FAK or $\beta$ actin-mRFP fusion proteins were cloned into the NheI /BamHI or HindIII/NotI sites of the pcDNA5/FRT vector. The host cells were then co-transfected with pOG44 plasmid encoding Flp recombinase and either pcDNA5/FRT-Paxillin-GFP, pcDNA5/FRT-GFP-FAK or pcDNA5/FRT-ßactin-mRFP, and recombinant cell lines were selected according to the manufacturer's instructions. Stable clones were analyzed for expression of paxillin-GFP, GFP-FAK and $\beta$ actin-mRFP 
by fluorescent microscopy and western blotting. Myc-tagged PDZ-RhoGEF, p115RhoGEF and (561-585) PDZ-RhoGEF-GFP plasmids were engineered as described elsewhere (Banerjee and Wedegaertner, 2004). Paxillin-GFP was a generous gift from Dr. Rick Horwitz, University of Virginia, and GFP-FAK was generated by cloning FAK from pCMV-myc -FAK vector (Xiong and Parsons, 1997) into pEGFP-C1 vector (Clontech, CA). Bactin-mRFP was a generous gift from the laboratory of Frank Getler (MIT, Cambridge,MA). Rho effector domain mutants were generous gifts from Dr. Kodi Ravichandran, University of Virginia. FAK, ROCKI and ROCK II shRNA plasmids were obtained from Sigma (USA) shRNA library. Lentiviral packaging plasmids pMDG and pCMVSR 8.2 were generous gifts from Dr. David Rekosh (University of Virginia). Lentiviral production was performed according to Sigma protocol. The organization of adhesions in cells knocked down for expression of FAK, PDZ-RhoGEF, ROCK I and ROCK II were assessed by immunostaining for FAK and paxillin.

\section{Serum starvation and LPA stimulation experiments.}

Serum deprivation is defined as removal of serum up to 6 hours. Serum starvation is defined as removal of serum for 12-16 hours. Rat 2 or NIH3T3 cells were plated over night on fibronectin $(1 \mu \mathrm{g} / \mathrm{ml})$ coated glass $\mathrm{T}$ dishes or plastic dishes in the presence of medium containing $10 \%$ serum. The following day, cells were provided medium containing serum, serum-free medium or serum free medium plus LPA $(2 \mu \mathrm{M})$. 


\section{Western Blotting.}

Cells were lysed in $200 \mu \mathrm{l}$ of RIPA buffer (50mM HEPES, $\mathrm{pH} 7.4,1 \%$ Triton X-100, 1\% sodium deoxycholate, $0.1 \% \mathrm{SDS}, 0.1 \mathrm{M} \mathrm{NaCl}, 1 \mathrm{mM}$ sodium orthovanadate, $0.1 \mathrm{M}$ sodium pyrophosphate, $100 \mathrm{mM} \mathrm{NaF}$ and $1 \mathrm{mM}$ PMSF). Lysates were clarified by centrifugation at 13,000 rpm for 10 minutes. Clarified lysates were boiled in 1x sample buffer (0.04 M Tris-HCl pH 6.8, 1\% SDS, 1\% $\beta$-mercaptoethanol and 10\% glycerol) for 10 minutes and resolved by SDS-PAGE. Proteins were transferred to Immobilon membrane (Whatman) and blocked with 5\% BSA in PBS (140 mM NaCl, $0.27 \mathrm{mM}$ KCL, $0.43 \mathrm{mM} \mathrm{Na}_{2} \mathrm{HPO}_{4} 7 \mathrm{H}_{2} \mathrm{O}, 0.14 \mathrm{mM} \mathrm{KH}_{2} \mathrm{PO}_{4}$, $\mathrm{pH}$ 7.3), 0.1\% Tween 20, pH 7.2 for 1 hour at room temperature. The membranes were incubated overnight at $4^{\circ} \mathrm{C}$ with one of the following antibodies: anti-actin (Zymed, 1:5000 dilution), anti-FAK polyclonal antibody (UpstateBiochemicals, 1:1000 dilution), anti-pY397 FAK polyclonal antibody (Biosource, 1:1000 dilution), anti-MAPK monoclonal antibody (1:2000, Sigma), antiphospho- MAPK polyclonal antibody (1:2000, gift from Dr. Michael J. Weber, University of Virginia), anti-GTRAP 48 (PDZRhoGEF) monoclonal antibody (1:1000, BD Transduction), anti-GFP polyclonal antibody (1:1000, Cell Signaling), anti-tubulin monoclonal antibody (1:5000, Sigma), anti-phospho tyrosine monoclonal antibody 4G10 (1:1000, UpstateBiochemicals) or anti-myc monoclonal antibody 9E10 (SantaCruz, 1:1000), polyclonal anti - ROCK II antibody (1:1000, Upstate), monoclonal anti-ROCK I/II (1:1000, Upstate) or monoclonal anti-Paxillin antibody (1:1000, BD Transduction.) Membranes were subsequently probed with secondary antibodies linked to HRP (Amersham). Western blot membranes were developed using enhanced chemiluminescent substrate for detection of HRP (Amersham). Western blot results were prepared using AlphaEaseFC (FluorChem 8800) for Windows software. 


\section{Immunoprecipitation experiments.}

Twenty four hours after transient transfection with indicated expression vectors cells were washed with ice cold PBS, and lysed in Immuno-Precipitation (IP) buffer (50 mM Tris-HCl, $\mathrm{pH}$ 7.6, $160 \mathrm{mM} \mathrm{NaCl}, 0.5 \mathrm{mM}$ EDTA/EGTA, 1\% Triton-X100, 10\% Glycerol, $1 \mathrm{mM}$ PMSF and $1 \mu \mathrm{g} / \mathrm{ml}$ Leupeptin). Cell extracts were clarified by centrifugation at $13,000 \mathrm{rpm}$ and cell supernatants were applied to agarose beads $(10 \%$ of total volume) conjugated with anti-myc 4A6 antibody (UpstateBiochemicals Inc). Immunoprecipitation was carried out for 2 hours at $4^{0} \mathrm{C}$ and immune complexes were washed 3 times with IP buffer and boiled in 1X sample buffer. Samples were processed for SDS-PAGE and western blotting as described above.

\section{Analysis of adhesion movement.}

Adhesion movements were visualized in serum deprived NIH3T3- or Rat2-Paxillin-GFP cells before and after the addition of $2 \mu \mathrm{M}$ LPA using TIRF microscopy. The cells were maintained at $37^{\circ}$ with a Bioptechs Delta T heated chamber system. Cells were imaged using a Nikon Eclipse TE2000-E inverted microscope equipped with TIRF illuminator, a 488 argon laser and a 60x DIC TIRF objective (NA 1.45) equipped with a Bioptechs

objective heater. Images were acquired with a Retiga 1300i CCD camera and the QCapture Pro software (Q Imaging). Adhesion movement was quantified as described before (Smilenov et al., 1999); briefly the first frame of the movie was pseudo-colored green and the last frame was pseudo-colored red, and the two images were merged. Adhesions that contained both red and green and thus appeared yellow were scored as 
poorly dynamic. The fraction of adhesions that moved was scored, determining the percentage of red adhesions relative to total adhesions in the first frame.

\section{Phase microscopy.}

NIH3T3-ßactin-mRFP and Rat2 cells were serum deprived and filmed in the absence or presence of $2 \mu \mathrm{M}$ LPA using Zeiss Axiovert $135 \mathrm{TV}$ microscope equipped with the CCD camera MTI 176.

\section{Immunostaining of cells.}

Control, FAK or PDZ-RhoGEF siRNA-treated Rat2 fibroblasts were plated overnight on fibronectin-coated coverslips ( $1 \mu \mathrm{g} / \mathrm{ml}$, Sigma) in media containing $10 \%$ serum. Cells were either left untreated or serum starved overnight and then stimulated with, $2 \mu \mathrm{M}$ LPA with $0.1 \%$ carrier BSA or $10 \%$ FBS for indicated times. Cells were fixed with $4 \%$ paraformaldehyde in PBS, permeabilized with $0.5 \%$ Triton X-100 in PBS and blocked with $20 \%$ goat serum in PBS. The cells were washed 3 times with PBS and immunostained with the following antibodies: anti-tubulin B2-11 (Sigma, 1:5000 dilution) and anti-myc 9E10 (1:250, SantaCruz). To visualize actin cells were treated with AlexaFluor 488 conjugated phalloidin (Invitrogen, Inc). To visualize microtubules or myc-tagged GEF constructs cells were treated with secondary goat anti-mouse antibodies conjugated to AlexaFluor 594 (1:2000, Invitrogen, Inc). Fluorescent images were acquired using a Nikon Eclipse E600 upright fluorescence microscope equipped with a Hamamatsu ORCA CCD camera. Images were captured and analyzed with OpenLab (Improvision) software. 


\section{Analysis of trailing edge length and cell elongation.}

To measure trailing edge length, a line was drawn from the tip of the trailing edge towards the base of the nucleus. To measure cell length, a line was drawn from the center of the nucleus to the furthest visible microtubule tip. To measure cell width, a second line was drawn across the cell passing through the center of the nucleus at a $90^{\circ}$ angle to the first line. To calculate the "elongation factor", the length was divided by the width.

\section{Analysis of lamellipodia protrusion.}

NIH3T3 cells stably expressing $\beta$ actin-mRFP or Rat2 cells were plated in medium containing $10 \%$ serum on fibronectin-coated $(1 \mu \mathrm{g} / \mathrm{ml})$ glass $\mathrm{T}$ dishes at the density of $3 \times 10^{4}$ cells/dish. The next day, serum-containing medium was changed to serum free medium or serum free medium containing $2 \mu \mathrm{M}$ LPA. After 6 hours cells were filmed for a total of 15-20 min, collecting one image every 20 seconds using TIRF (NIH3T3) or DIC (Rat2) microscopy. Protrusion rates were calculated using Image J software (Wayne Rasband, National Institute of Health. USA).

\section{Statistics.}

Statistical analysis was done using non-parametric, non assuming Gaussian distribution Mann-Withnay t-test using Prism software. The symbols denote the following statistical differences: $\quad * * * \quad \mathrm{p}=0.001 ; \quad * * \quad \mathrm{p}=0.01 ; \quad * \quad \mathrm{p}=0.05$. 


\section{Results}

\section{LPA stimulates trailing edge retraction and rear adhesion dynamics.}

NIH3T3 cells, cultured on fibronectin and deprived of serum for 6 hours, acquired a distinct morphology characterized by a protrusive lamellipodia and an elongated tail-like structure, which was approximately four times longer than control cells deprived of serum but then treated with LPA (Fig. 9). Interestingly, the rate of lamellipodia protrusion was the same in cells without serum and stimulated with LPA (Fig. 9). Cells serum starved overnight (12-16 hours) also displayed an elongated phenotype with significantly less protrusive activity (Fig. 10). Stimulation of serum starved NIH3T3 cells with $2 \mu \mathrm{M}$ LPA stimulated the retraction of the elongated tail and restored normal cell morphology whereas addition of $100 \mathrm{nM}$ PDGF was much less effective at inducing normal morphology (Fig. 3). Time lapse imaging of serum starved cells stimulated with $2 \mu \mathrm{M}$ LPA revealed that the restoration of normal rounded morphology occurred with a $t_{1 / 2}$ of 90 to $120 \mathrm{~min}$ (Fig. 11). To assess whether the LPA induced trailing edge retraction was reflected in changes in the dynamic behavior of adhesions, TIRF microscopy was used to monitor Paxillin-GFP labeled adhesions in cells serum starved over night and in LPA stimulated cells. As shown in Fig. 12, adhesions present in the rear of serum starved cells did not exhibit a change in either fluorescence intensity or position indicating that these adhesions were relatively stable. In contrast, LPA stimulation significantly increased the dynamics of the adhesions as measured by the inward movement of the adhesions over a 7 minute time period (Fig. 12). As previously reported the addition of LPA to serum starved cells induced a rapid increase in the tyrosine phosphorylation of FAK (Fig. 13A, B). These data indicate that LPA 
signaling is sufficient to induce changes in the movement of mature adhesions found in the trailing edge and that the activation of FAK is concomitant with this process.

\section{The lack of FAK expression in fibroblasts results in an elongated phenotype in the presence of serum and failure to induce adhesion movement and trailing edge retraction.}

The knock down of FAK using either siRNAs designed to specifically target rat FAK (Tilghman et al., 2005) or shRNA RNA to specifically target mouse FAK resulted in an elongated cell morphology (Fig. 14A,B) when cells were grown in the presence of serum. To confirm that the observed elongation of the cells was due to the loss of FAK expression, a Rat 2 cell line was created that constitutively expressed (at approximately $20 \%$ the level of endogenous FAK) GFP-tagged chicken FAK that was not targeted by the FAK siRNA (Fig. 15A). The morphology of cells expressing GFP-FAK was indistinguishable from control cells (Fig. 15B). While FAK siRNA treatment increased cell elongation in control Rat2 cells, the GFP-FAK expressing cells retained their more rounded morphology (Fig. 15B). FAK immunostaining revealed that in control cells FAK silencing resulted in the absence of endogenous FAK in adhesions (Fig. 15B). In contrast, FAK siRNA treatment of Rat2 cells expressing GFP-FAK did not decrease the expression of GFP-FAK or alter its localization to adhesions (Fig. 15B). Therefore, the changes in cellular morphology were a consequence of reduced FAK expression and correlated with the loss of FAK from cell matrix adhesions.

To examine whether FAK functions downstream of LPA to stimulate adhesion dynamics and trailing edge retraction, Rat2 cells that stably expressed paxillin-GFP (Rat2 Paxillin-GFP) were treated with control or FAK siRNA. Cells were serum starved for 
twelve hours and stimulated with $2 \mu \mathrm{M}$ LPA and adhesion dynamics were measured using TIRF microscopy (Fig. 16). LPA readily induced dynamic movement of adhesions in control cells but failed to do so in FAK siRNA-treated cells (Fig. 16). This result is consistent with the failure of FAK siRNA treated cells to retract trailing edge in response to LPA. Consistent with these observations, addition of serum or LPA to serum starved control cells induced restoration of cell shape whereas serum starved FAK knockdown cells largely maintained their elongated morphology in response to either serum or LPA (Fig. 17). These observations are consistent with LPA controlling trailing edge retraction and changes in cell shape through a receptor pathway requiring FAK activation and downstream signaling.

\section{Over expression of specific Rho pathway components rescues FAK knock down phenotype.}

To determine the downstream effectors for FAK that contribute to adhesion movement and trailing edge retractions, we assessed the role of RhoGEFs and putative Rho effectors. The RhoGEF, PDZ-RhoGEF has been shown to be an important effector of the LPA receptor signaling pathway (Yamada et al., 2005). To investigate the role of this GEF, Rat2 cells treated with FAK siRNA to block FAK expression were transfected with myc-tagged PDZ-RhoGEF, PDZ-RhoGEF lacking Rho DH domain (PDZ-RhoGEF 1585) and full length p115RhoGEF. The expression level of each of the Rho GEFs was similar (Fig. 18A) and the localization of GEFs was consistent with previously published data (Banerjee and Wedegaertner, 2004). Both PDZ-RhoGEF and PDZ-RhoGEF 1-585 but not p115RhoGEF localized to actin cytoskeleton (data not shown). As shown in Fig 18B the expression of wild type PDZ-RhoGEF but not PDZ-RhoGEF 1-585 or full length 
p115RhoGEF reversed changes in cell shape induced by FAK siRNA treatment of Rat 2 cells. These data indicate that overexpressed full length PDZ-RhoGEF bypasses requirement for FAK in regulation of adhesion dynamics necessary for rear retraction and restoration of cell shape.

To assess which putative Rho effectors maybe important for the restoration of cell shape in FAK deficient NIH3T3 cells, we expressed two different active Rho effector domain mutants (Palazzo et al., 2001; Tosello-Trampont et al., 2003) in NIH3T3 cells with dowregulated FAK expression (Fig.19A). As shown in Fig. 19B expression of the GFP-tagged Rho effector mutant that can activate both mDia and ROCK (RhoG14VF39V) efficiently rescued the elongated phenotype induced by FAK deficiency. In contrast, the negative control GFP-PDZ-RhoGEF (561-585) a non functional GEF with actin localization or the Rho effector mutant that activates only mDia (RhoG14VF39A) failed to rescue the elongated phenotype in FAK deficient cells (Fig. 19B). These data indicate that FAK and PDZ-RhoGEF cooperate to activate the Rho/ROCK pathway and induce trailing edge retraction.

\section{PDZ-RhoGEF-Rho-ROCK pathway is necessary for LPA induced trailing edge retraction and stimulation of adhesion dynamics}

We next sought to determine whether PDZ-RhoGEF forms a complex with FAK and localizes to FAK containing adhesions. Myc-PDZ-RhoGEF 1-585 or myc-p115RhoGEF was over expressed along with GFP-FAK in HEK293T cells. As shown in Fig.20A, GFPFAK was readily detected in immune complexes with myc-PDZ-RhoGEF 1-585 but not in immune complexes with myc-p115RhoGEF. To test if FAK and PDZ-RhoGEF colocalized in adhesions we over expressed myc-tagged PDZ-RhoGEF, PDZ-RhoGEF 1- 
585 and p115RhoGEF in Rat2 cells stably expressing GFP-FAK. We observed that mycPDZ-RhoGEF, myc-PDZ-RhoGEF 1-585 but not myc-p115RhoGEF localized to FAK containing adhesions (Fig. 20B).To confirm the requirement for PDZ-RhoGEF in a signaling pathway that controls cell elongation downstream of the LPA receptor, Rat2 cells were treated with PDZ-RhoGEF siRNA to attenuate PDZ-RhoGEF expression (Fig. 21A). PDZ-RhoGEF siRNA treated cells exhibited elongated phenotype even in the presence of serum (Fig. 21B, phase images); a phenotype comparable to that observed in FAK siRNA treated cells. Stimulation of serum starved control and PDZ-RhoGEF siRNA treated cells with $2 \mu \mathrm{M}$ LPA revealed that the PDZ-RhoGEF siRNA treated cells remained elongated (Fig. 21C). TIRF microscopy was used to measure adhesion dynamics after LPA stimulated of serum starved control and PDZ-RhoGEF siRNA treated Rat2 -Paxillin-GFP cells (Fig. 22A). As shown in Fig. 22B LPA stimulation of control cells but not PDZ-RhoGEF siRNA treated cells accelerated adhesion dynamics. These data provide additional support for the role of PDZ-RhoGEF in a FAK-dependent pathway that contributes to the regulation of rear retraction and adhesion movement.

Since a Rho effector domain mutant that can not activate ROCK failed to rescue FAK knockdown phenotype we reasoned that shRNA mediated knockdown of ROCK I /II would prevent LPA from stimulating adhesion movement and trailing edge retraction in response to LPA. As shown in Fig. 23 A, shRNA mediated knockdown of ROCK II but not ROCK I expression induced cell elongation in the presence of serum. The phenotype of ROCKII knockdown cells however was distinct from that observed for FAK knockdown cells (shown in Figure 14AB) in that the cell bodies exhibit a somewhat flatter morphology. In contrast the knock down of ROCK I significantly impaired formation of mature adhesions and actin stress fibers (Figure 24 and data not shown) consistent with recent observations (Totsukawa et al., 2004; Yoneda et al., 2005). We 
observed that LPA failed to induce adhesion movement in cells lacking either ROCK I or ROCK II (figure 24A,B) indicating that both ROCK I and II contribute to the regulation of adhesion mobility. As shown in Fig. 25 knockdown of ROCK II prevented the LPAdependent stimulation of trailing edge retraction and concomitant decrease in cell elongation. These results support a model in which LPA activation of $\mathrm{G}_{12 / 13}$ leads to activation of FAK/PDZ-RhoGEF/Rho/ROCK II pathway that regulates the dynamics of mature adhesions leading to trailing edge retraction and concomitant decrease in cell elongation in serum starved cells treated with LPA.

\section{Discussion.}

In this work we show that serum deprivation of rodent fibroblasts markedly reduced trailing edge retraction without having significant effect on lamellipodia protrusive activity at the leading edge. The perturbation in trailing edge retraction results in an elongated cell morphology, following over night serum starvation. Addition of LPA to serum starved cells stimulates rear retraction and decreases cell elongation and provides a model that parallels events within the trailing edge of a migrating fibroblast. Importantly, trailing edge retraction requires LPA induced stimulation of the dynamic movement of mature adhesions. Using this model, we have identified specific signaling components that contribute to trailing edge retraction in fibroblasts in response to LPA. LPA induced retraction of the trailing edge requires FAK. The siRNA mediated attenuation of FAK expression blocks the LPA induction of adhesion dynamics, trailing edge retraction and restoration of cell shape, suggesting that dynamic remodeling of adhesions is necessary for trailing edge retraction. LPA-induced changes in adhesion dynamics; trailing edge retraction and cell elongation also required PDZ-RhoGEF. The 
siRNA mediated knockdown of PDZ-RhoGEF blocked LPA induced adhesion dynamics and prevented the restoration of cell shape. Furthermore, expression of PDZ-RhoGEF rescues FAK siRNA phenotype with respect to cell elongation (cells are less elongated). In addition PDZ-RhoGEF co-immunoprecipitated with FAK and co-localizes with FAKcontaining adhesions suggesting that the activation of FAK and PDZ-RhoGEF are closely coupled. Finally, two lines of evidence suggest that one of the major targets of activated Rho in response to LPA is ROCKII. Expression of a Rho effector mutant that preferentially activates $\mathrm{mDia}$ failed to rescue the elongated phenotype of FAK knockdown cells; whereas a mutant that activates both mDia and ROCK pathways rescued the elongated phenotype of FAK siRNA treated cells. In addition the knockdown of ROCKII but not ROCK I induced a phenotype that parallels that observed in FAKdeficient cells with respect to cell elongation and retention of stress fibers and adhesions structures (Table 1). Our observations are consistent with a model in which LPA binding to its receptor stimulates activation of $\mathrm{G}_{12 / 13}$, activation of FAK and the closely coupled activation of PDZ-RhoGEF, Rho and ROCKII, leading to spatial (trailing edge) stimulation of adhesion dynamics via localized increase in contractility, retraction of the cell rear and decrease in cell elongation (Figure 26).

There is abundant evidence that G protein-coupled receptors (GPCRs), such as those for LPA, activate Rho and Rho-dependent signaling pathways through the $\mathrm{G}_{12 / 13}$ family of heterotrimeric G proteins (Barr et al., 1997; Gohla et al., 1998; Majumdar et al., 1999; Offermanns et al., 1994). The RhoGEFs, p115RhoGEF , PDZRhoGEF and LARG (leukemia-associated RhoGEF) contain a regulator of G protein signaling (RGS) domain that binds activated $\mathrm{G}_{\alpha 12 / 13}$ resulting in RhoGEF activation (Wang et al., 2004). Thus, the RGS-RhoGEFs are thought to serve as effectors of activated $\alpha 12 / 13$ and as molecular 
bridges between the heterotrimeric G proteins and the Rho network. (Fukuhara et al., 1999; Reuther et al., 2001; Togashi et al., 2000; Zheng et al., 1995b).

A variety of studies show that activation of tyrosine kinases by GPCRs is required for the efficient stimulation of Rho and Rho signaling [for review see (Sah et al., 2000)]. Indeed, many GPCRs are potent activators of FAK, suggesting a close link between FAK activation and Rho signaling (Della Rocca et al., 1999). Furthermore, it has been reported that stimulation of $\mathrm{G}_{12 / 13}$ induces $\mathrm{FAK}$ dependent PDZ-RhoGEF tyrosine phosphorylation leading to enhanced activation of Rho (Chikumi et al., 2002). How FAK is participating in $G_{12 / 13}$ signaling to Rho remains unclear. The observation that FAK forms a complex with PDZ-RhoGEF and PDZ-RhoGEF has been reported to oligomerize (Chikumi et al., 2004), leads us to speculate that LPA mediated activation of $\mathrm{G}_{12 / 13}$ may induce oligomerization of PDZ-RhoGEF and concomitant dimerization of FAK, leading to transphosphorylation and activation of FAK within such complexes. Alternatively, LPA stimulation of acidic phospholipids (PIP2) may activate FAK directly, as has been recently demonstrated by (Cai et al., 2007). We speculate that activated FAK may phosphorylate PDZ-RhoGEF stimulating GEF activity towards Rho and that this localized activation of Rho might lead to activation of effectors at or proximal to adhesion sites.

Previously it has been shown that LPA stimulation of serum-starved neuronal cells treated with ROCK inhibitor resulted in the failure of LPA to induce cell shape changes, rapid neurite retraction and cell rounding (Jalink et al., 1993; Kranenburg et al., 1999). However, this study did not delineate the contribution of ROCK I and ROCK II in the process of LPA induced neurite retraction. More relevant to the current study are the observations that knock down of ROCK II but not ROCK I yielded a cellular phenotype similar to that observed following FAK knock down with respect to cell shape, 
augmented stress fibers and loss of dynamic adhesions (Yoneda et al., 2005). In addition, recent studies showed that Rho dependent breakdown of cell-cell adhesions requires ROCK II but not ROCK I (Samarin et al., 2007). Therefore, both studies point to a role for ROCK II in the induction of adhesion complex dynamics/breakdown. Recent evidence (Yoneda et al., 2005) indicates that ROCK I rather than ROCKII is important for stress fiber formation. ROCK II appears to act to regulate microfilament bundling at the adhesion site and is involved in phagocytosis of matrix-coated beads, a function not sensitive to ROCK I depletion.

The effects of ROCK II on adhesion movement in trailing edge of fibroblasts could be attributed to induction of localized activation of myosin. We speculate that ROCKII-dependent tension at the trailing edge adhesions during LPA stimulation would contribute to the force that would promote adhesion inward movement and ultimate disassembly. In cells with attenuated FAK, PDZ-RhoGEF or ROCKII expression, adhesions remained stable even after LPA stimulation, indicating that this pathway is important in regulating localized changes in contractility. This observation would suggest that adhesion turnover requires localized modulation of myosin activity provided by the localized activation of the FAK-GEF-Rho-ROCKII pathway. 


\section{Figures and Tables}

Figure 9. Serum starvation of NIH3T3 cells results in the failure of trailing edge retraction.

Length of the trailing edge and protrusion rates in NIH3T3-ßactin-mRFP cells in the absence of serum and in the absence of serum plus $2 \mu \mathrm{M}$ LPA. For trailing edge measurement, cells were filmed for 6 hours immediately after serum removal with images acquired at 5 minutes intervals, using phase contrast microscopy. Images acquired at the end of the sixth hour are shown and quantified. The data represents the analysis of 60 cells from two different experiments. Images acquired at the end of $6^{\text {th }}$ hour are shown. For protrusion of the lamellipodia, cells were starved for $6 \mathrm{hrs}$ and at the end of 6th hour, images of $\beta$-actin-mRFP in protruding lemellipodia were obtained for 20 minutes at 20 second intervals. The data represent analysis of 6 protruding lamellipodia per experimental condition. 
Figure 9
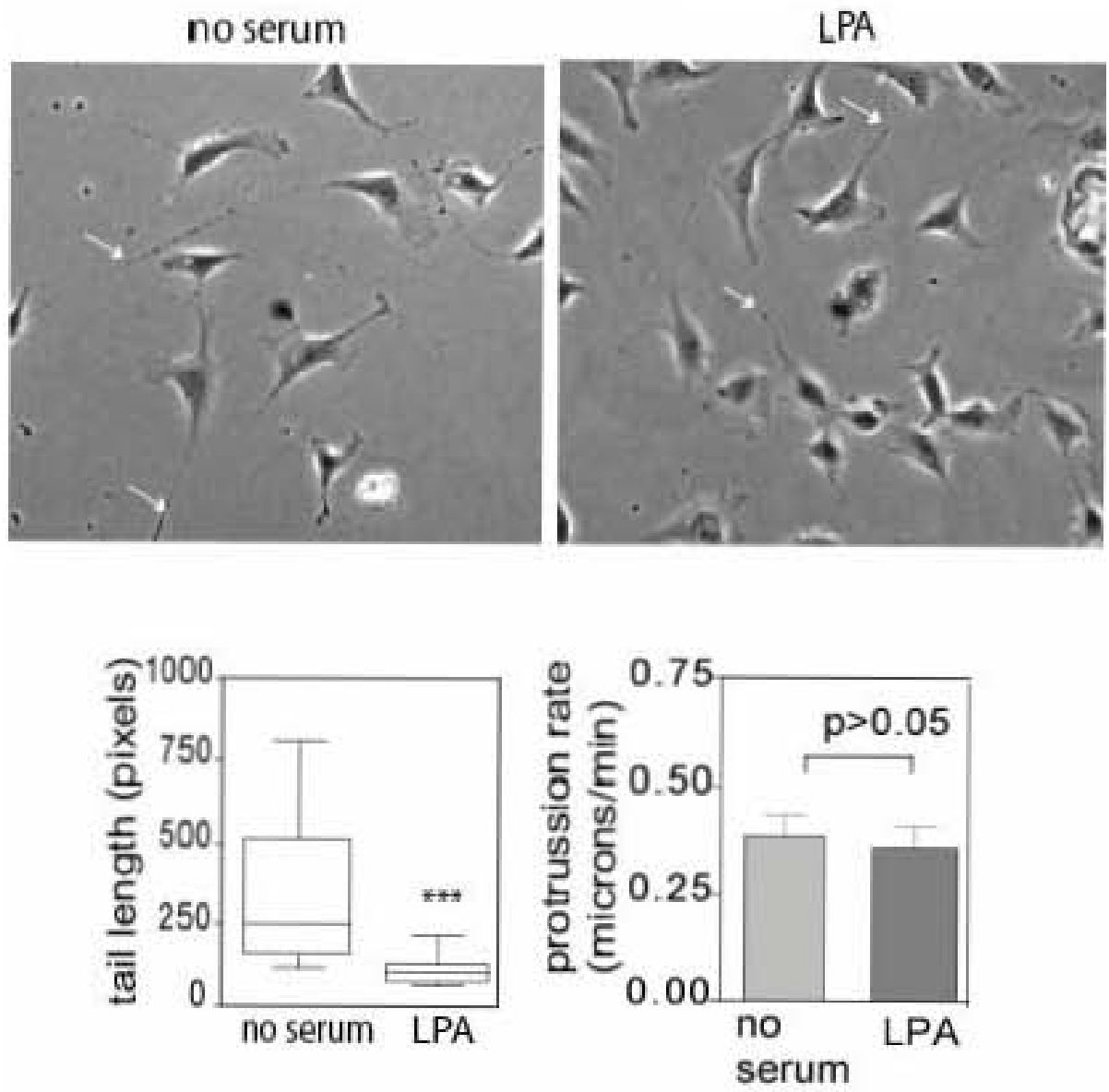
Figure 10. Failure of NIH3T3 cells to retract trailing edges results in cell shape changes that depend on LPA but not PDGF.

Phase contrast images and quantification of cell elongation of serum starved cells, serum starved cells treated with $2 \mu \mathrm{M}$ LPA or $100 \mathrm{nM}$ PDGF. The data represent the analysis of 50-72 cells per condition from two different experiments. 
Figure 10
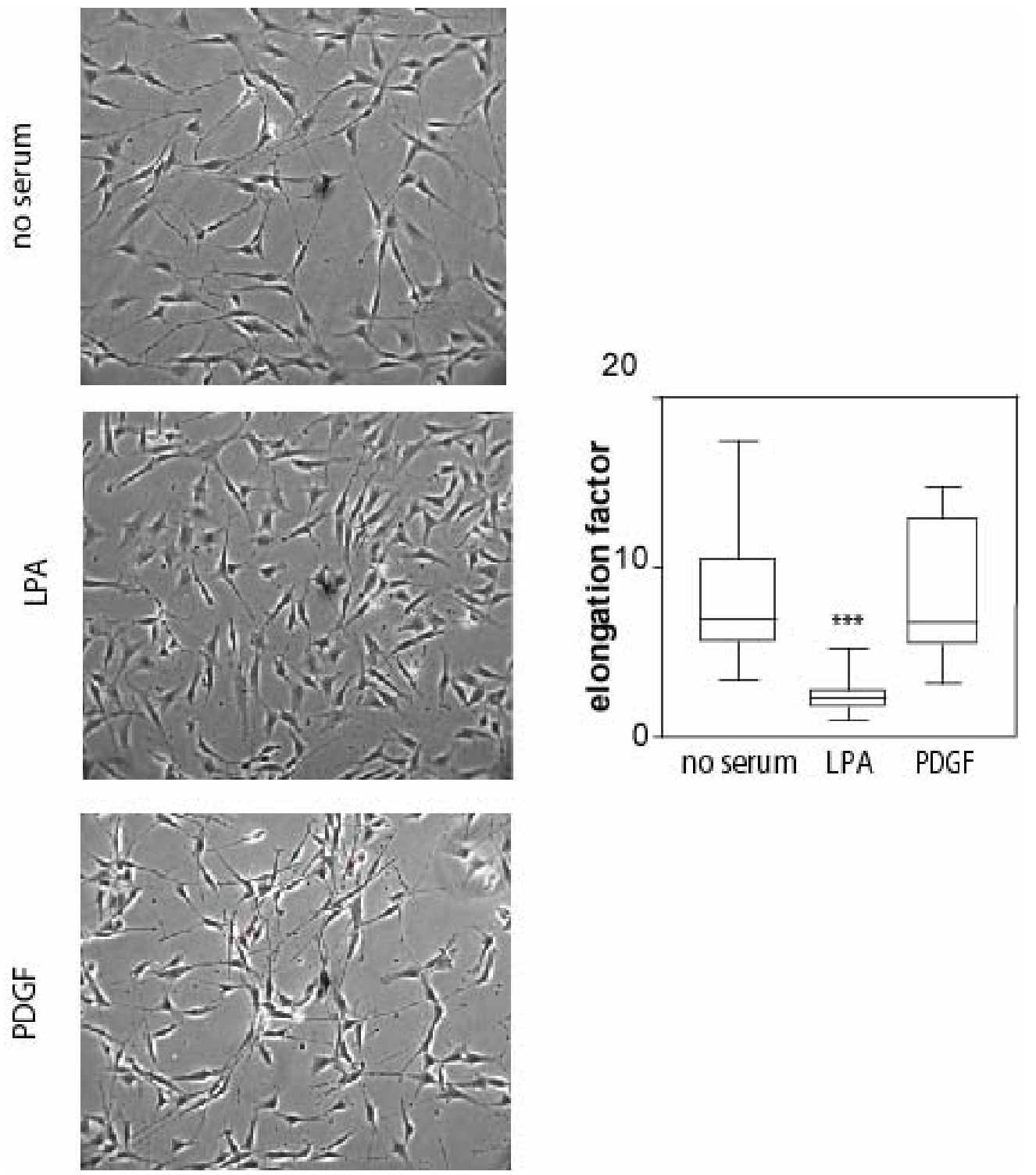
Figure 11. LPA induces trailing edge retraction in serum deprived NIH3T3 cells Kinetics of changes in cell elongation upon LPA stimulation of serum starved fibroblasts. 
Figure 11

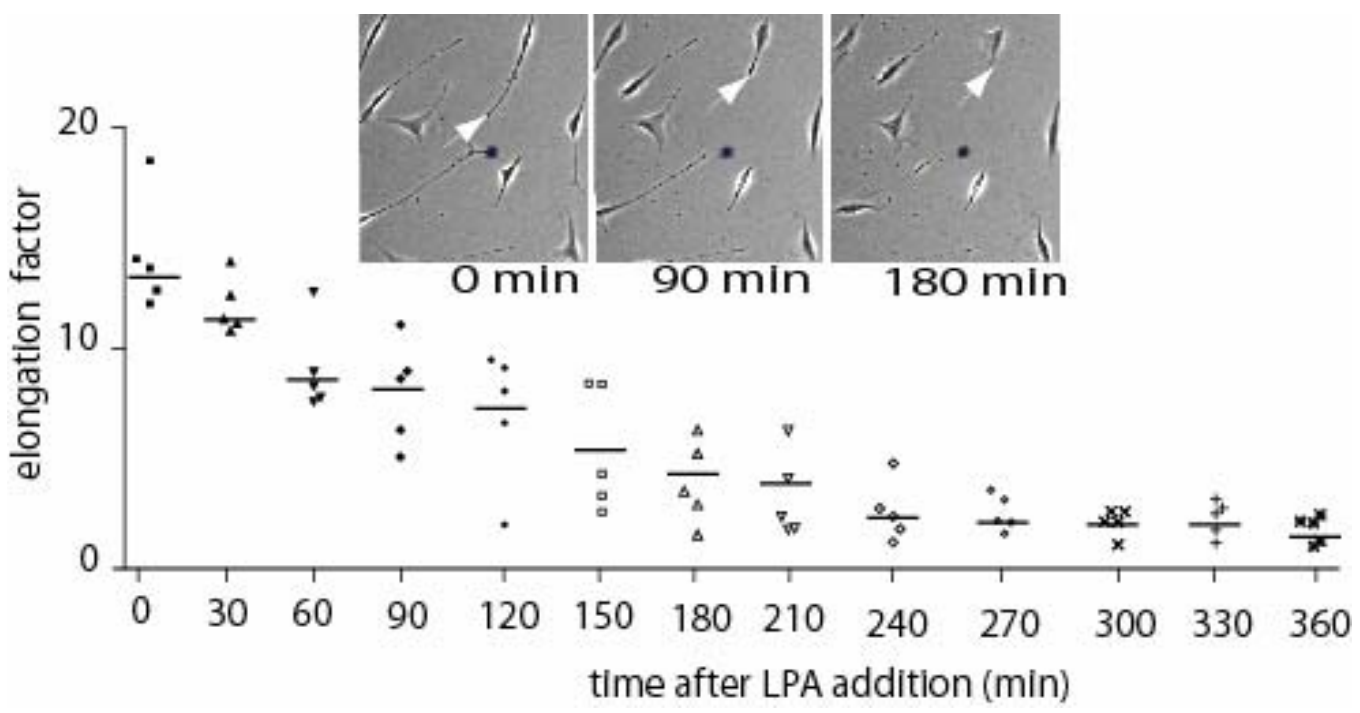


Figure 12. LPA stimulates movement of adhesions found in the trailing edge of NIH3T3 cells.

NIH3T3 cells stably expressing Paxillin GFP were plated in the presence of serum containing medium (10\%) on dishes ciated with $1 \mathrm{ug} / \mathrm{ml}$ fibronectin. The following day cells were placed in serum free medium for 12 hours and subsequently stimulated with 2 $\mu \mathrm{M}$ LPA and the movement of adhesions was analyzed using TIRF microscopy as described in Materials and Methods. Cells were filmed for 7 minutes prior to addition of LPA and 7 minutes after addition of LPA (arrows indicate representative adhesions). Bar graphs represent quantification of adhesion movement induced by LPA in NIH3T3 cells expressing Paxillin-GFP. 
Figure 12
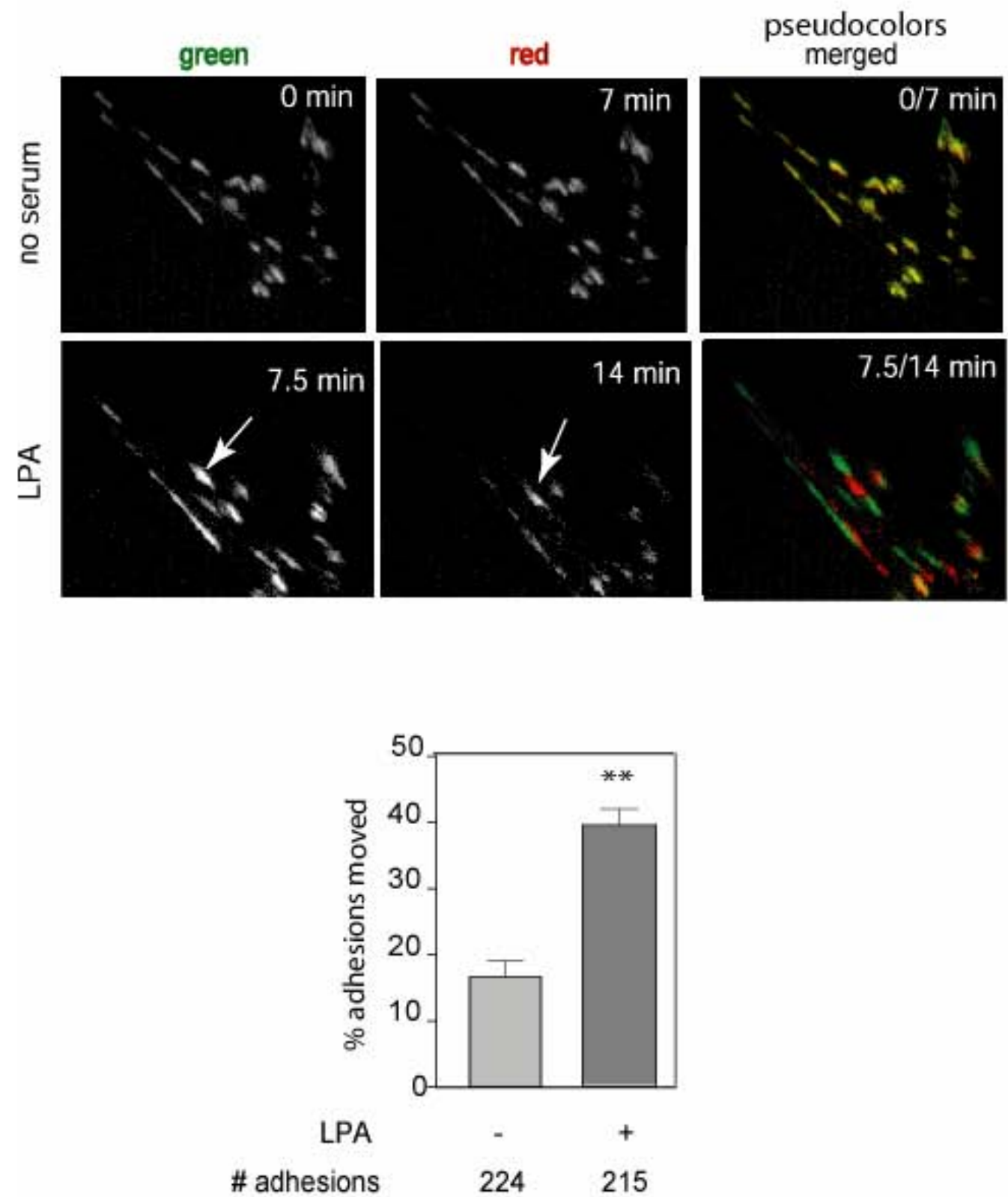
Figure 13. LPA stimulation of serum starved NIH3T3 and Rat 2 cells induces activation of Focal Adhesion Kinase.

(A) Western blot analysis of FAK activation by LPA in Rat 2 cells stably expressing GFP-FAK. Cells were stimulated with LPA for 5 minutes and GFP-FAK phosphorylation was assessed using anti-pTyr antibody as described in Materials and Methods. The increase in phosphorylation of ERK was determined by immunoblotting using antibodies against phosphorylated and total ERK (B) LPA activation of endogenous FAK in Rat 2 cells. Cells were stimulated with LPA for 5 minutes and western blots were probed with anti-pTyr397 or anti-FAK antibody as described in Materials and Methods 
Figure 13

(A)

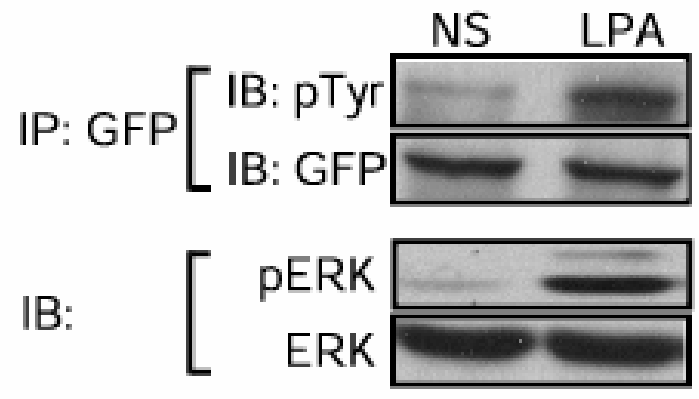

(B)

IB: $\left[\begin{array}{r}\text { NS } \quad \text { LPA } \\ \text { FAK FAK } \\ \end{array}\right.$


Figure 14. Knockdown of FAK expression induces cell elongation in the presence of $10 \%$ serum.

$(\mathbf{A , B})$ Phase contrast images, western blot analysis of FAK protein expression and quantification of cell elongation in control and FAK siRNA treated Rat2 cells and FAK shRNA treated NIH3T3 cells. Rat2 cells were treated with either siRNAs specific for FAK (A) or NIH 3 T3 cells were stably transfected with shRNA specific for FAK (B) as described in Materials and Methods. The extent of knockdown was determined by western blotting using FAK specific monoclonal antibodies and for the loading controls tubulin or paxillin specific antibodies. 
Figure 14
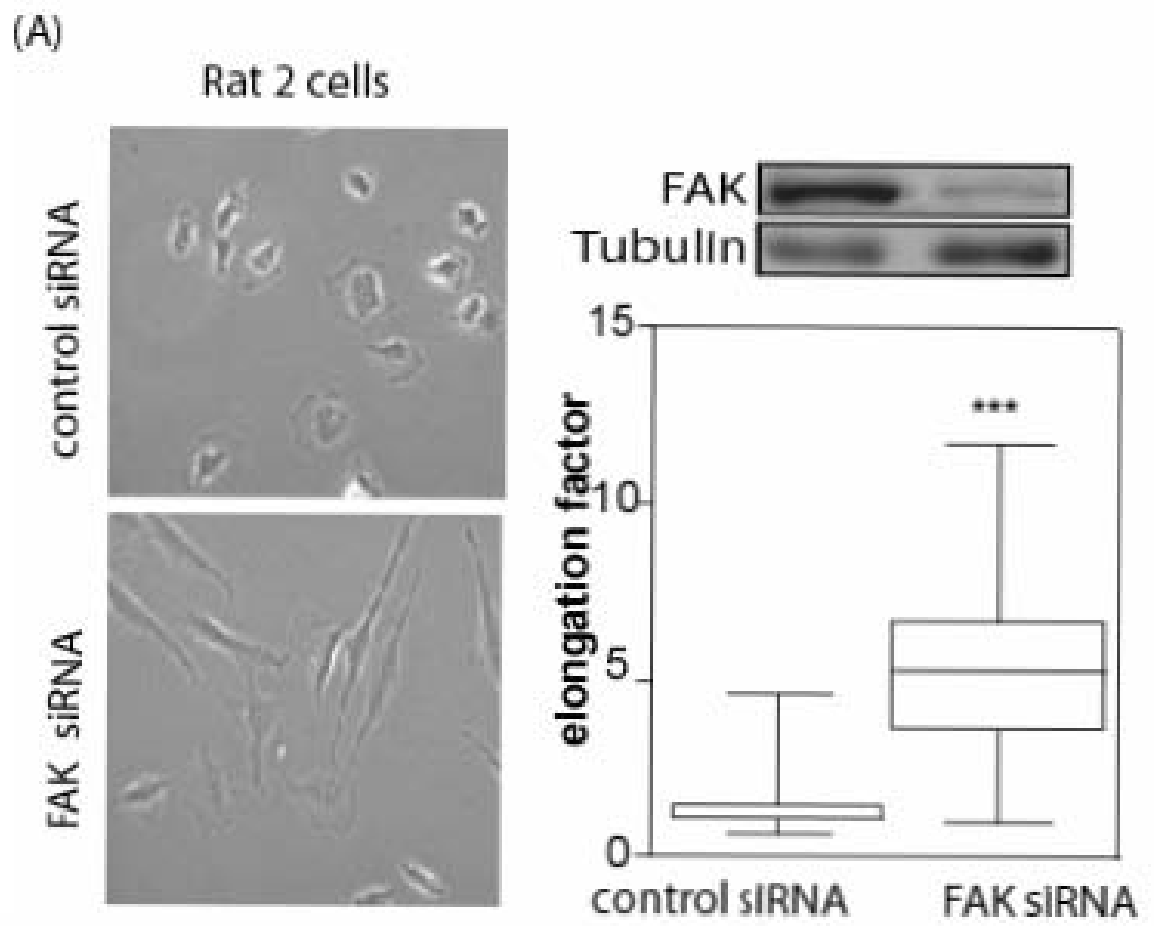

(B)
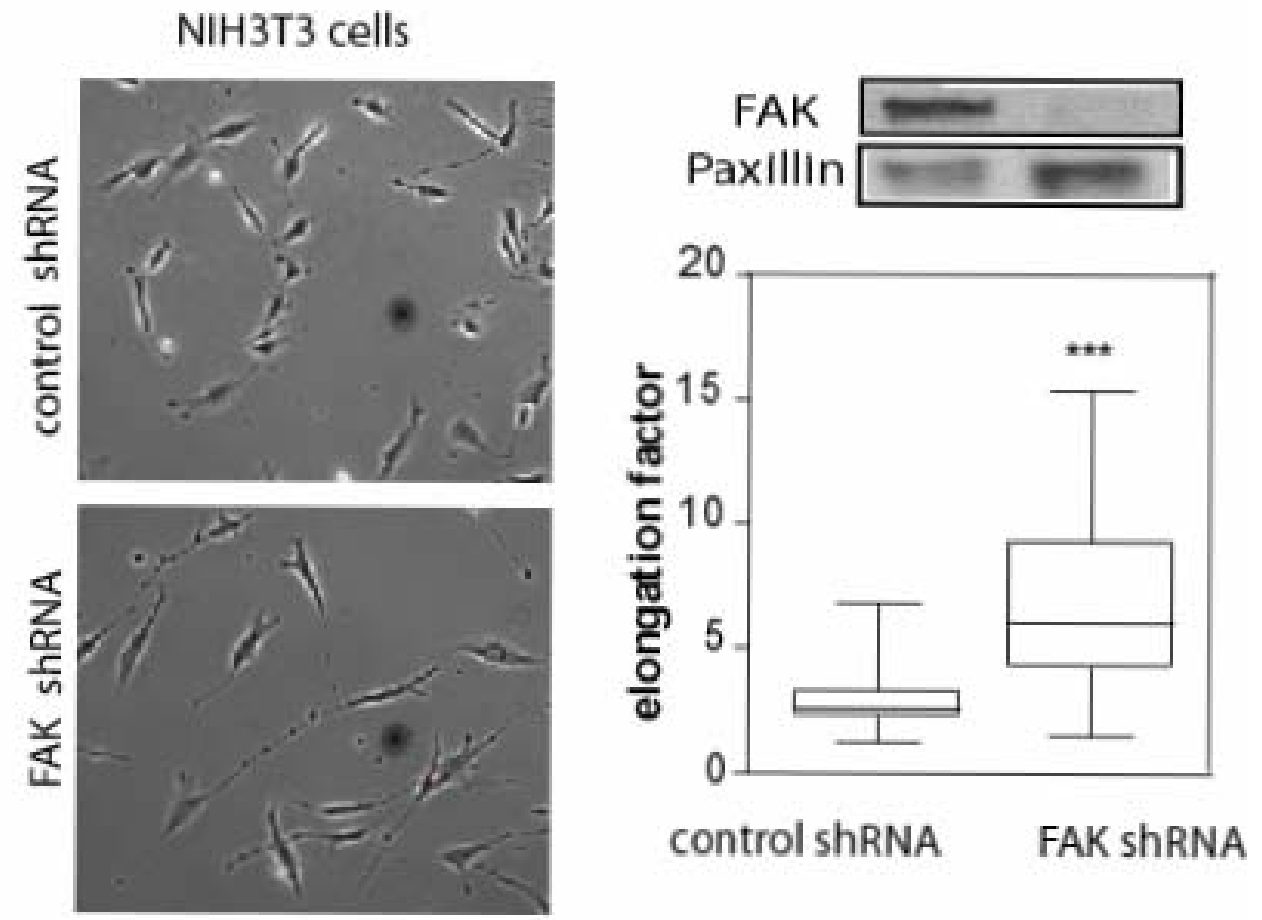
Figure 15. Re-expression of FAK in cells treated with FAK siRNA rescues the defect in cell elongation.

(A) Western blot analysis of FAK expression in wild type Rat 2 cells, Rat2 cells expressing LacZeo and Rat2 cells expressing GFP-FAK. (B) GFP-FAK expression decreases cell elongation resulting from FAK siRNA treatment. LacZeo expressing cells and GFP-FAK expressing cells were treated with control or FAK siRNA and stained with anti FAK(LacZeo) or left unstained to visualize GFP (GFP-FAK). The images illustrate the reduced FAK staining in LacZeo expressing Rat2 cells after FAK siRNA treatment but not control siRNA treatment (left). FAK siRNA treatment of GFP-FAK expressing cells did not alter GFP-FAK localization to adhesions (right panel). Control and FAK siRNA treated cells were immunostained with anti-tubulin (not shown) and the shape of individual cells was assessed by measuring the Elongation Factor. 
Figure 15

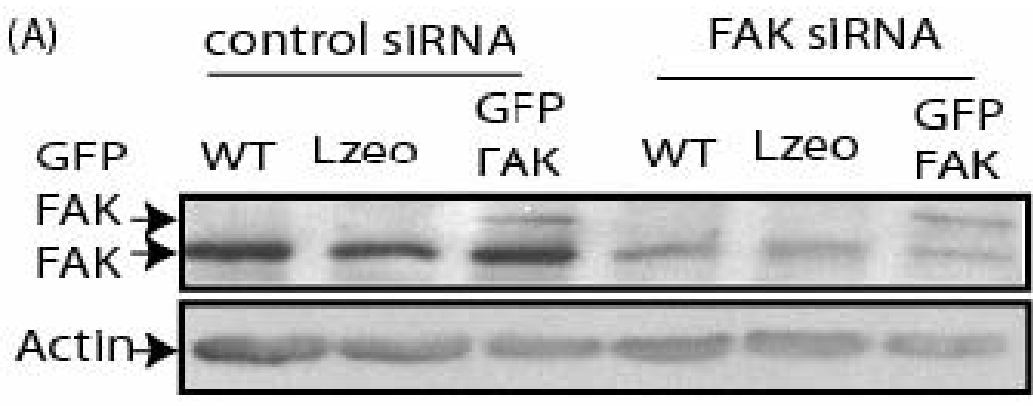

(B)
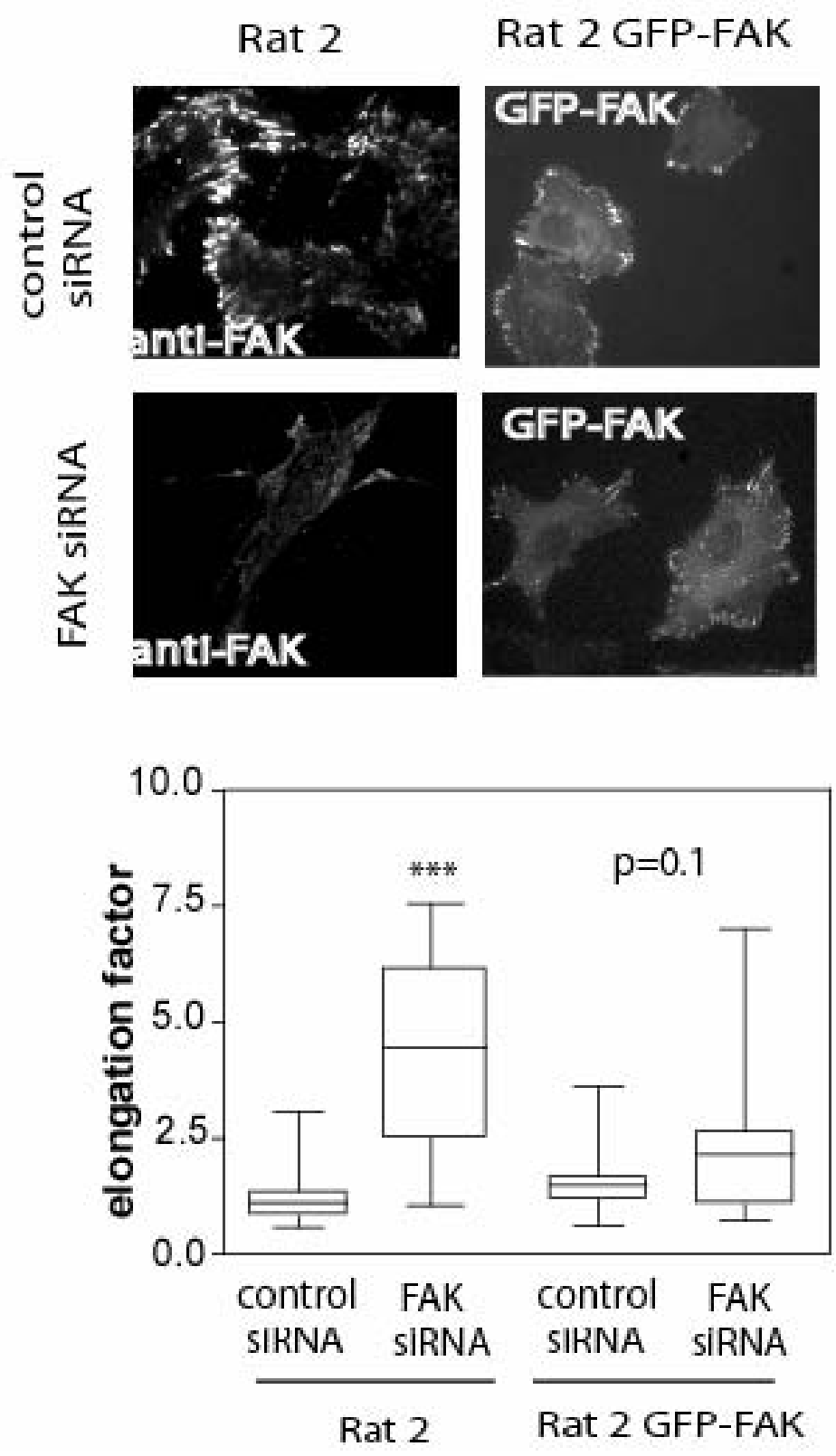


\section{Figure 16. LPA-induced adhesion movement is FAK-dependent}

Analysis of adhesion movement in response to $2 \mu \mathrm{M}$ LPA. Control or FAK siRNA treated Rat2 cells stably expressing Paxillin-GFP were plated on fibronectin coated $1 \mu \mathrm{g} / \mathrm{ml}$ glass $\mathrm{T}$ dishes in the presence of $10 \%$ serum containing medium. The following day, cells were serum starved for 12 hours and stimulated with $2 \mu \mathrm{M}$ LPA. Adhesion movement was analyzed using TIRF microscopy as described in Fig. 3. Cells were filmed for 27 minutes prior to the addition of LPA and 27 minutes after the addition of LPA. Bar graphs represent quantification of dynamic adhesions that was assessed as described in Fig. 5 (arrows indicate representative adhesions). The experiment was performed 3 times with 6 to 8 cells analyzed per experimental condition. 
Figure 16
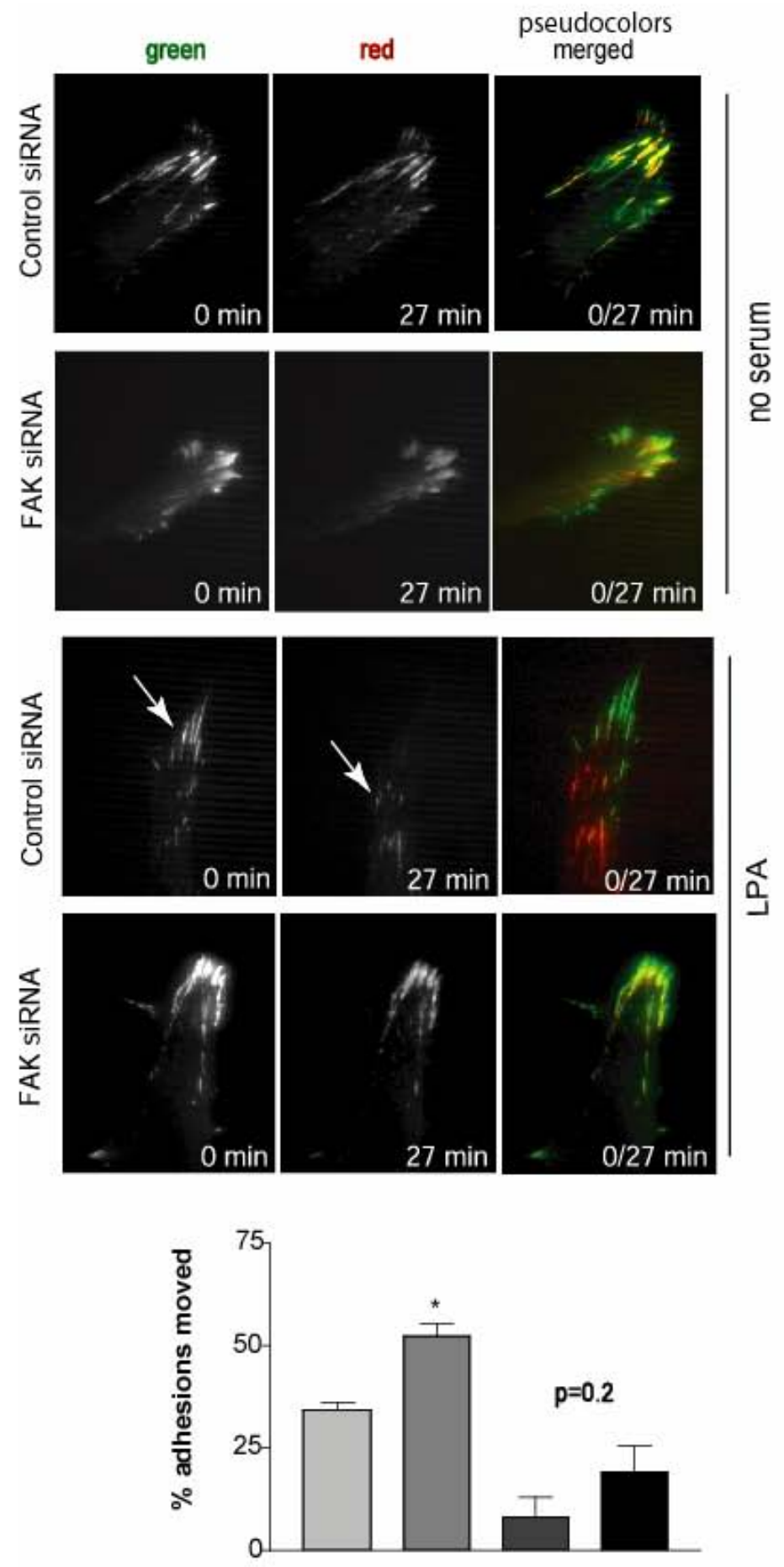

LPA

\# of adhesions

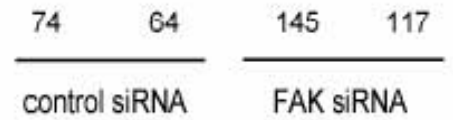


Figure 17. FAK is required for LPA-mediated induction of trailing edge retraction and the restoration of normal morphology.

Representative images of $\alpha$ tubulin immunostained control and FAK siRNA treated cells that were either serum starved or serum starved and stimulated with $10 \%$ serum or $2 \mu \mathrm{M}$ LPA are shown. The graphs show quantification of the elongation factor for siRNA treated cells following treatment of over night serum starved cells with serum or LPA for 7 hours. The experiment was repeated two times and 50 to 60 cells were analyzed per condition. 
Figure 17
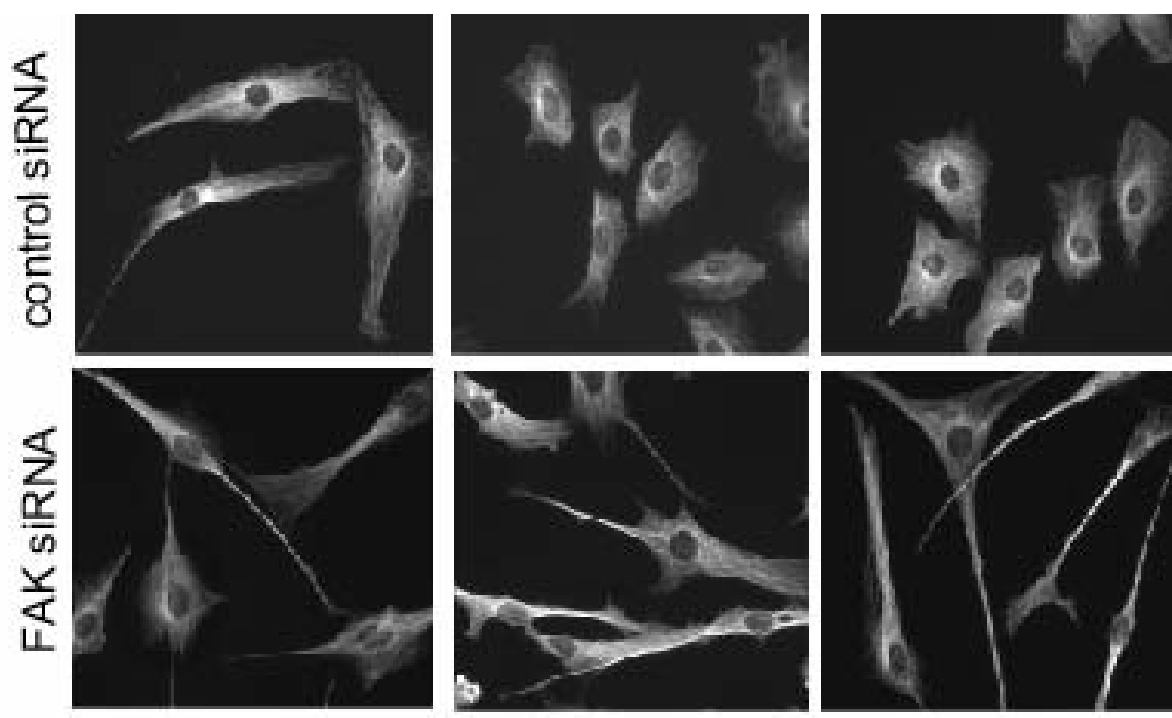

no serum

serum

LPA

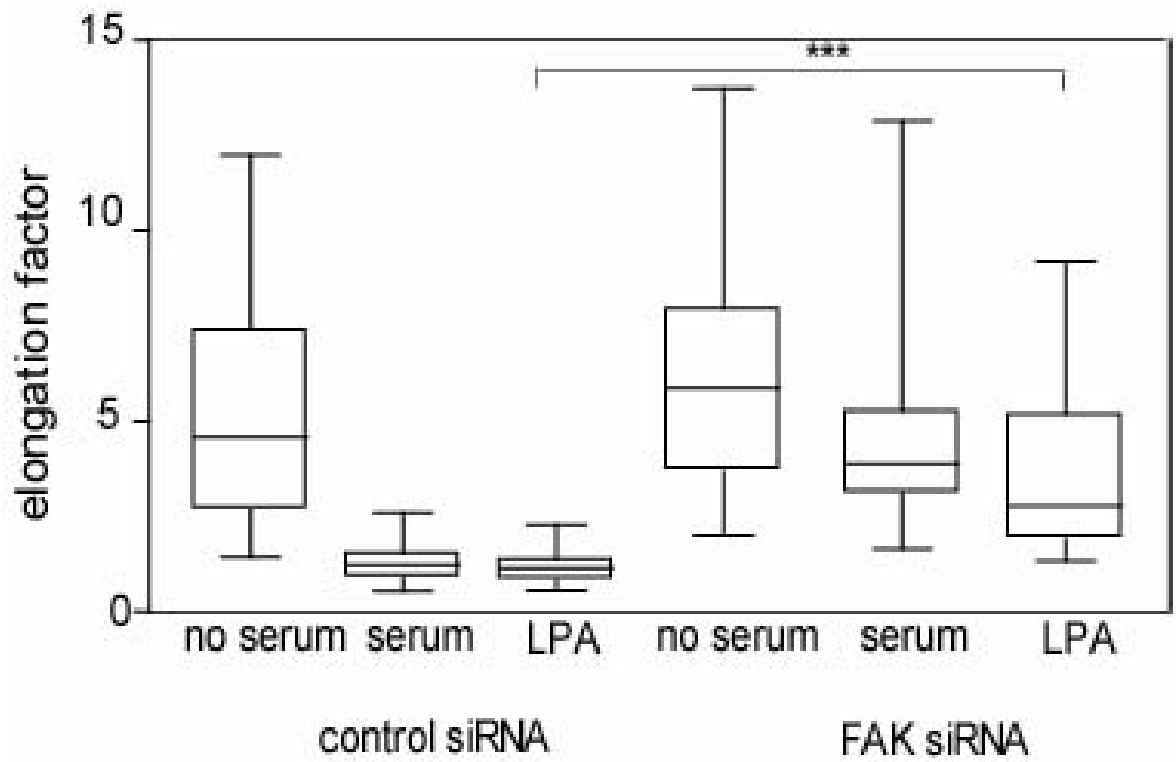


Figure 18. Ectopic expression of PDZ-RhoGEF rescues the elongation induced by knockdown of FAK.

(A) NIH3T3 cells were transiently transfected with PDZ-RhoGEF, p115RhoGEF and PDZ-RhoGEF 1-585, as described in Materials and Methods. Twenty four hours after transfection, lysates were prepared and blotted with anti myc antibody (B) Quantification of cell elongation in FAK siRNA treated cells expressing different Rho GEF molecules. 
Figure 18

(A)

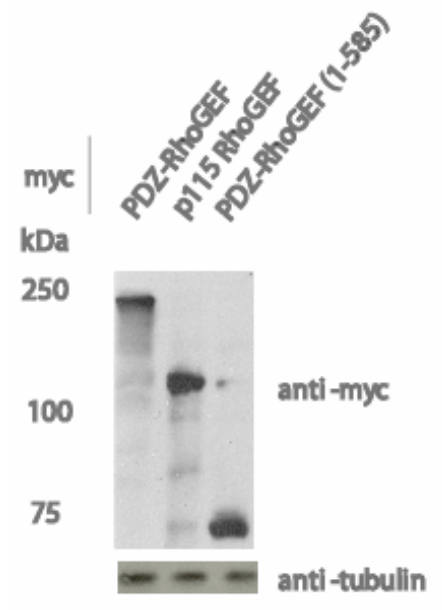

(B)

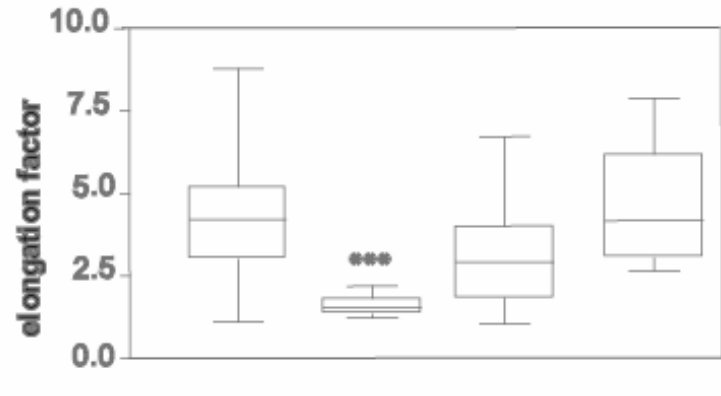

\begin{tabular}{|c|c|c|c|}
\hline number of cells & 50 & 20 & 25 \\
\hline non transfected & + & - & - \\
\hline PDZ-RhoGEF & - & + & - \\
\hline Y p1 15-RhoGEF & - & - & + \\
\hline $\begin{array}{c}\text { PDZ-RhoGEF } \\
(1-585)\end{array}$ & - & - & - \\
\hline
\end{tabular}


Figure 19. Ectopic expression of Rho effector domain mutant that activates ROCK but not mDia rescues the elongation induced by knockdown of FAK expression.

(A) FAK shRNA treated cells were transiently transfected with control, non-functional (561-585) PDZ-RhoGEF-GFP or G14VRho/F39A-GFP or G14VRho/F39V-GFP molecules as described in Materials and Methods. Twenty four hours after transfection, lysates were prepared and blotted with anti GFP antibody. (B) Quantification of cell elongation in FAK shRNA treated cells expressing different Rho effector domain mutant molecules. 
Figure 19

(A)

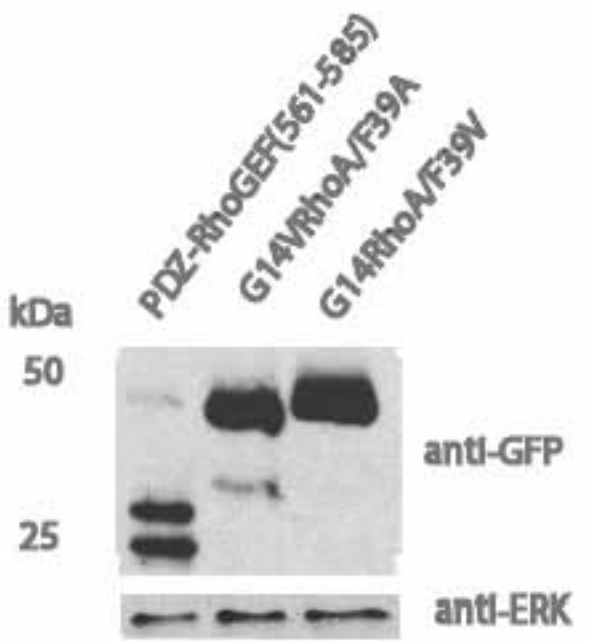

(B)

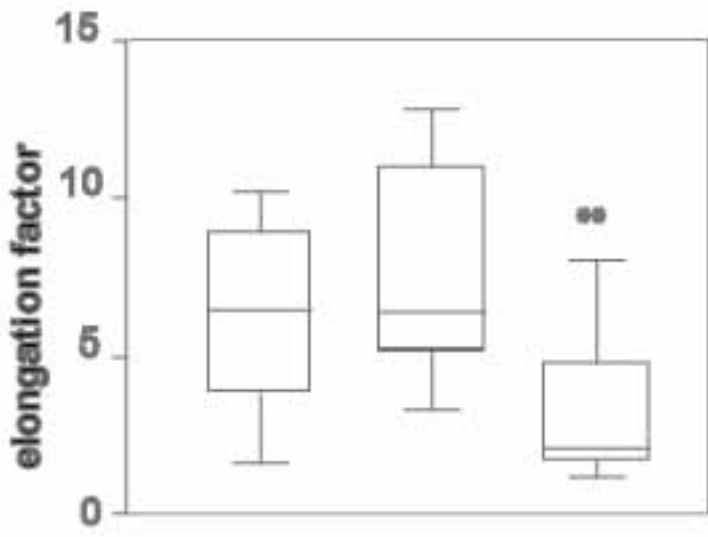

\begin{tabular}{|c|c|c|}
\hline number of cells & 61 & 32 \\
\hline (561-585)PDZ-RhoGEF & + & - \\
\hline RhoA G14V/F39A & - & + \\
\hline RhoA G14/F39V & - & - \\
\hline
\end{tabular}


Figure 20. GFP-FAK co-immunoprecipitates and localizes with co-expressed myctagged PDZ-RhoGEF and myc-tagged PDZ-RhoGEF 1-585.

(A) HEK293T cells were transfected with with $0.5 \mu \mathrm{g}$ GFP-FAK and myc-PDZ-RhoGEF 1-585 or myc-p115RhoGEF. Twenty four hours post transfection cells were processed for anti-myc immunoprecipitation, SDS PAGE and western bloting with anti GFP and anti FAK antibodies as described in Materials and Methods. (B) Rat2 cells stably expressing GFP-FAK were transiently transfected with myc-PDZ-RhoGEF, myc-PDZ-RhoGEF 1585 or myc-p115RhoGEF. Twenty four hours after transfection cells were detached and seeded on fibronectin coated $(1 \mu \mathrm{g} / \mathrm{ml})$ glass $\mathrm{T}$ dishes. Twenty four hours later, cells were fixed and immunostained with anti-myc antibody as described in Materials and Methods and visualized using TIRF microscopy. 
Figure 20

(A)

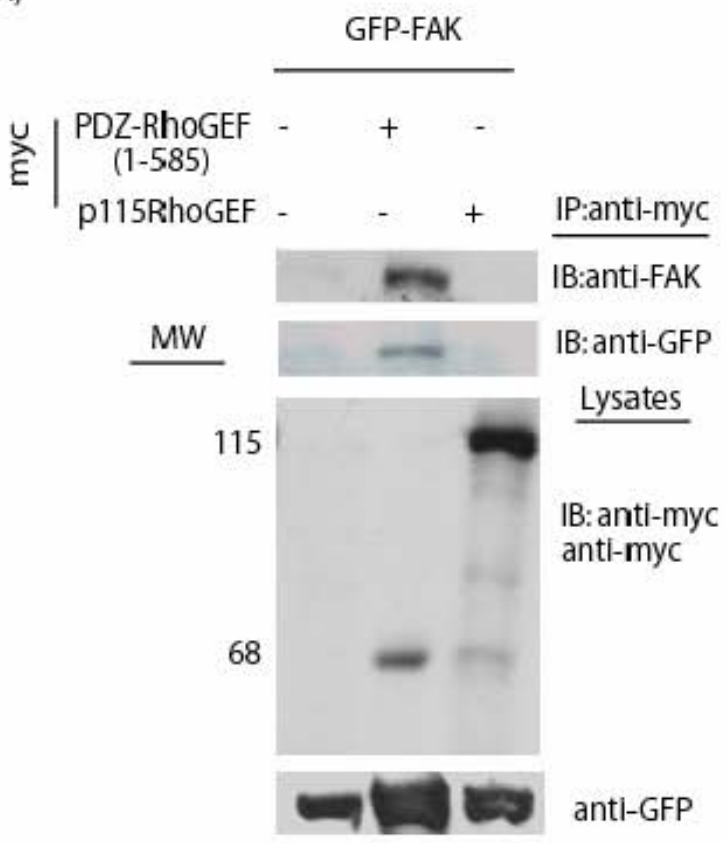

(B)

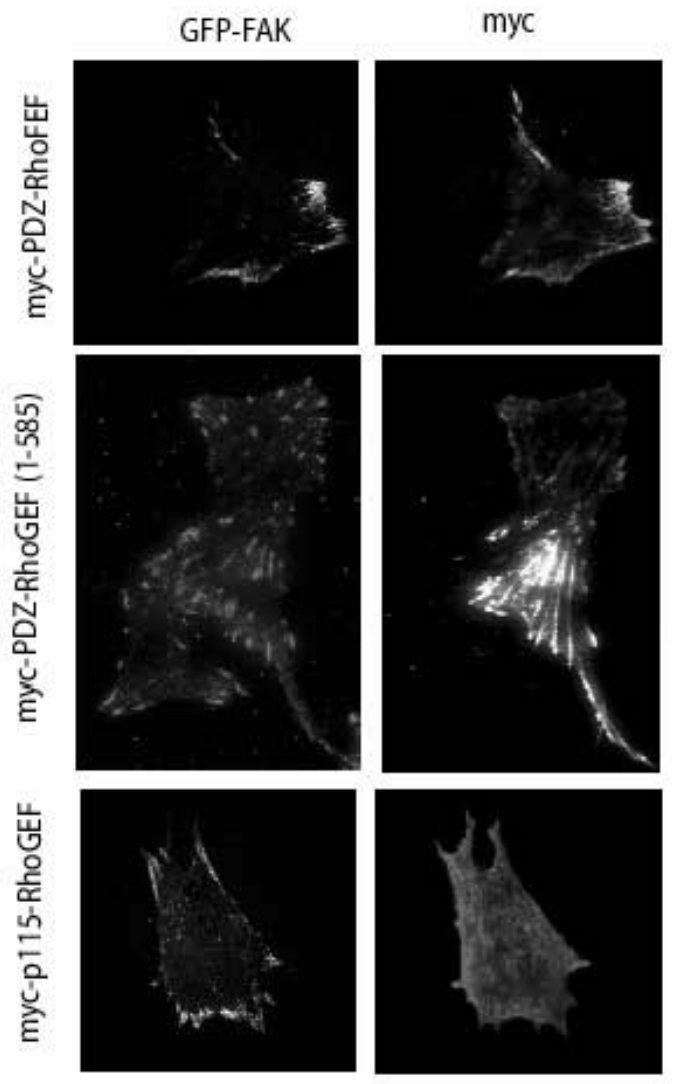


Figure 21 PDZ-RhoGEF is required for LPA stimulated trailing edge retraction.

(A) Western blot analysis of endogenous PDZ-RhoGEF (GTRAP48) protein expression and phase contrast images of control and PDZ-RhoGEF siRNA treated Rat2 cells. (B) fluorescent images and cell elongation quantification of control and PDZ-RhoGEF siRNA treated cells. Control or PDZ-RhoGEF siRNA treated cells were plated for 24 hours on fibronectin $(1 \mu \mathrm{g})$ coated microscope coverslips in the presence of $10 \%$ serum. Next day, cells were serum starved for 12-16 hours. Following starvation, cells were stimulated with $2 \mu \mathrm{M}$ LPA for 7 hours. Cells were fixed and stained with anti $\alpha$-tubulin antibody as described in Materials and Methods. 
Figure 21

(A)

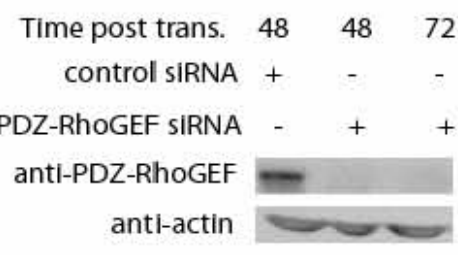

(B)
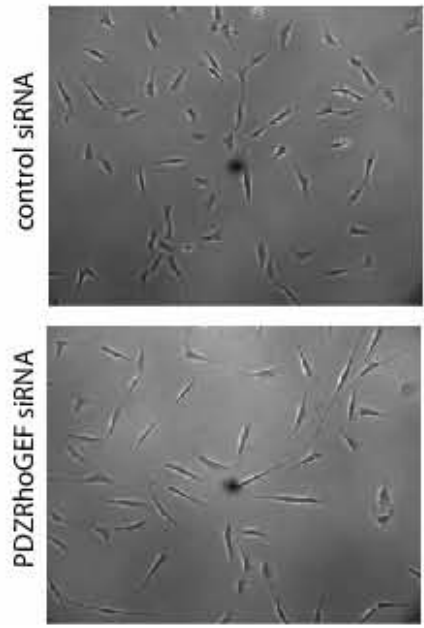

(c)

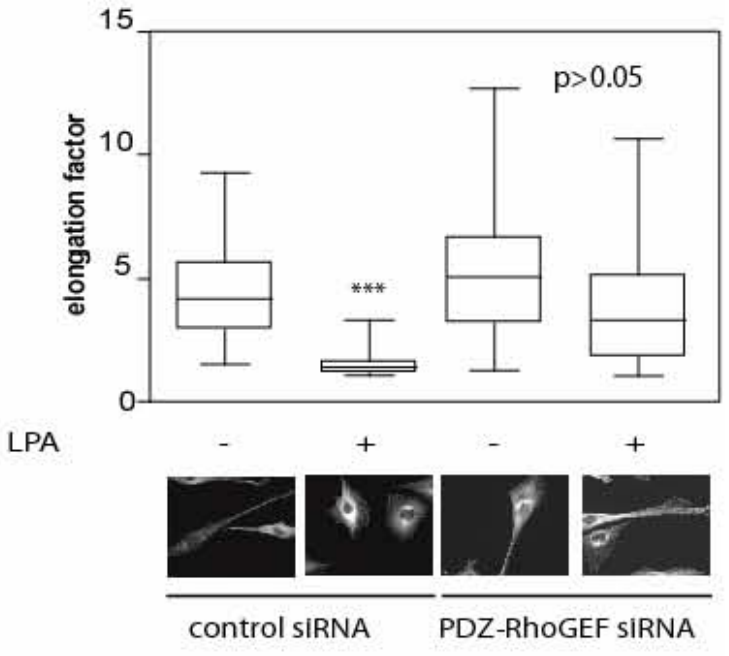


Figure 22. PDZ-RhoGEF is required for LPA induction of adhesion movement.

(A) Analysis of adhesion movement in response to LPA. Control or PDZ-RhoGEF siRNA treated Rat2 cells stably expressing Paxillin-GFP were plated on fibronectin coated $1 \mu \mathrm{g} / \mathrm{ml}$ glass $\mathrm{T}$ dishes in the presence of $10 \%$ serum containing medium and the following day cells were serum starved for 12-16 hours and then stimulated with $2 \mu \mathrm{M}$ LPA. Adhesion dynamic was analyzed using TIRF microscopy as described in Fig. 3. Cells were filmed for 21 minutes prior to the addition of $2 \mu \mathrm{M}$ LPA and 27 minutes after the addition of $2 \mu \mathrm{M}$ LPA. (B) Quantification of dynamic adhesions was assessed as described in Fig. 2 (arrows indicate representative adhesions). The experiment was performed 2 times with 5 to 7 cells analyzed per condition. 
Figure 22

(A)
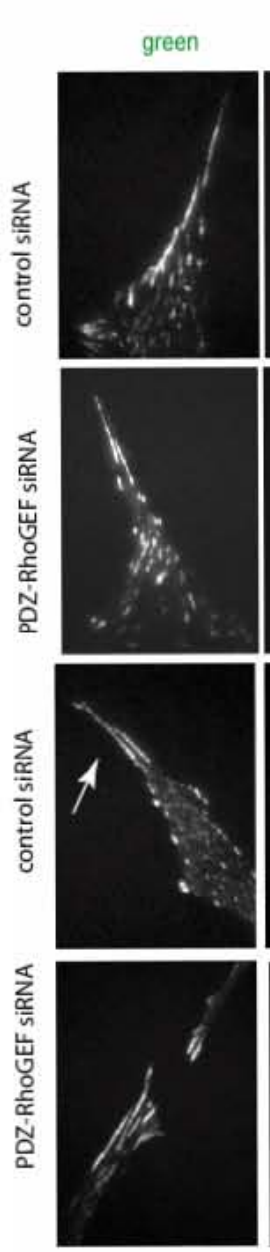

0 min
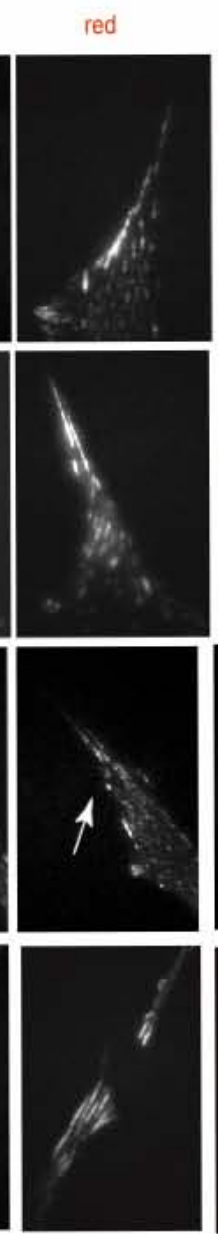

$27 \mathrm{~min}$
(B)

pseudocolors merged
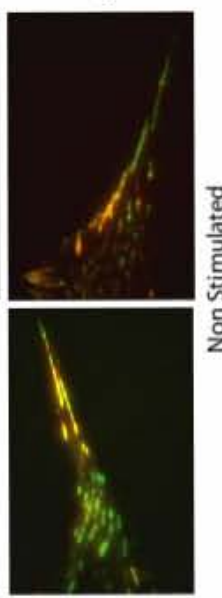

든 LPA

\# adhesions

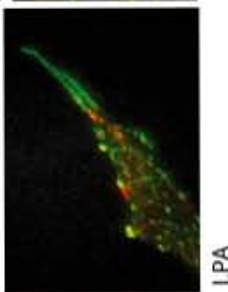

$\coprod$

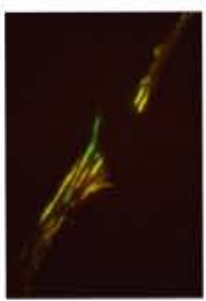

$0 / 27$

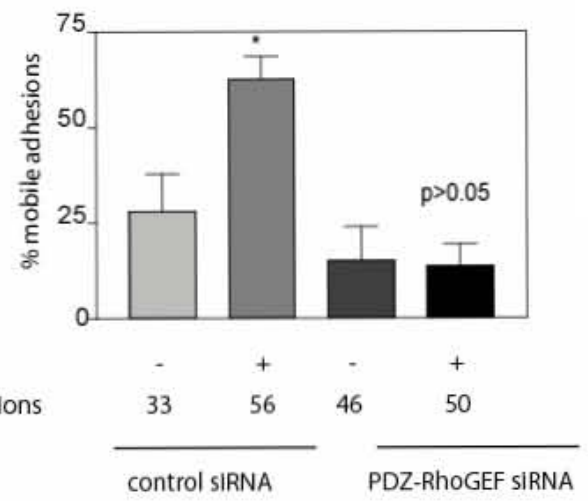


Figure 23. shRNA mediated attenuation of ROCK II but not ROCK I induces phenotype similar to FAK and PDZ-RhoGEF knock down.

Western blot analysis of ROCK I/II protein expression in control, FAK, ROCK I and ROCK II shRNA-treated NIH3T3 cells (top panel) and phase contrast images of control, ROCK I and ROCK II shRNA treated cells (bottom panel) 
Figure 23

(A)

shRNA control FAK Rock II Rock I

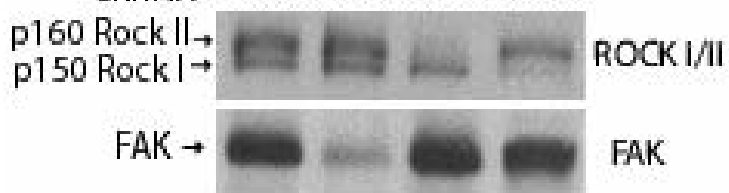

(B)
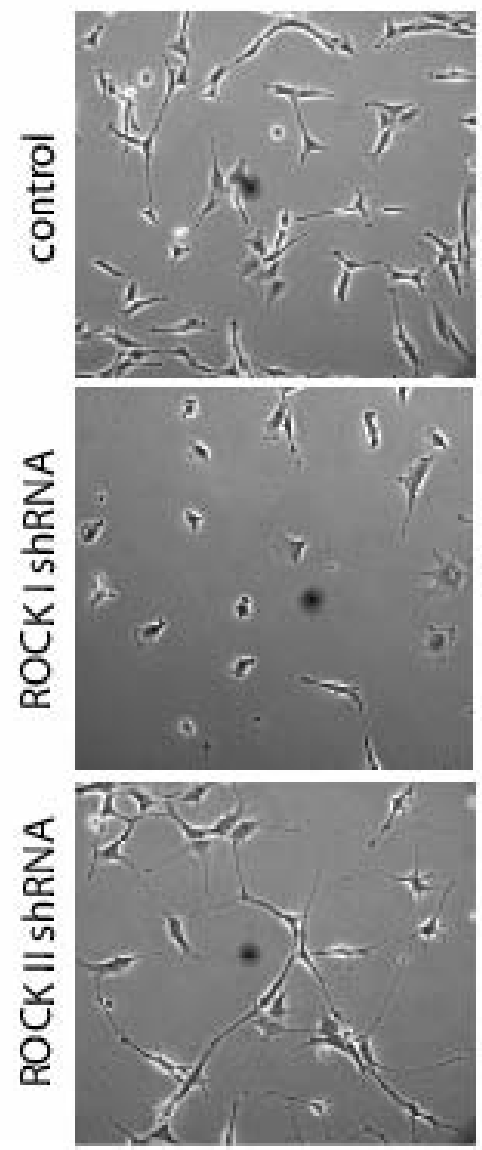
Figure 24. LPA-induced adhesion movement requires both ROCK isoforms.

(A) Control ROCK I and ROCK II shRNA treated cells were serum starved for 12 hours. Following the starvation, cells were stimulated with $2 \mu \mathrm{M}$ LPA, and the adhesion movement was visualized by TIRF microscopy. (B) Quantification of adhesion movement (as described in Materials and Methods) in serum starved cells and serum starved cells stimulated with LPA (arrows indicate representative mobile adhesions). The experiment was performed 2 times with 6-8 cells analyzed per experimental condition. 
Figure 24

(A)
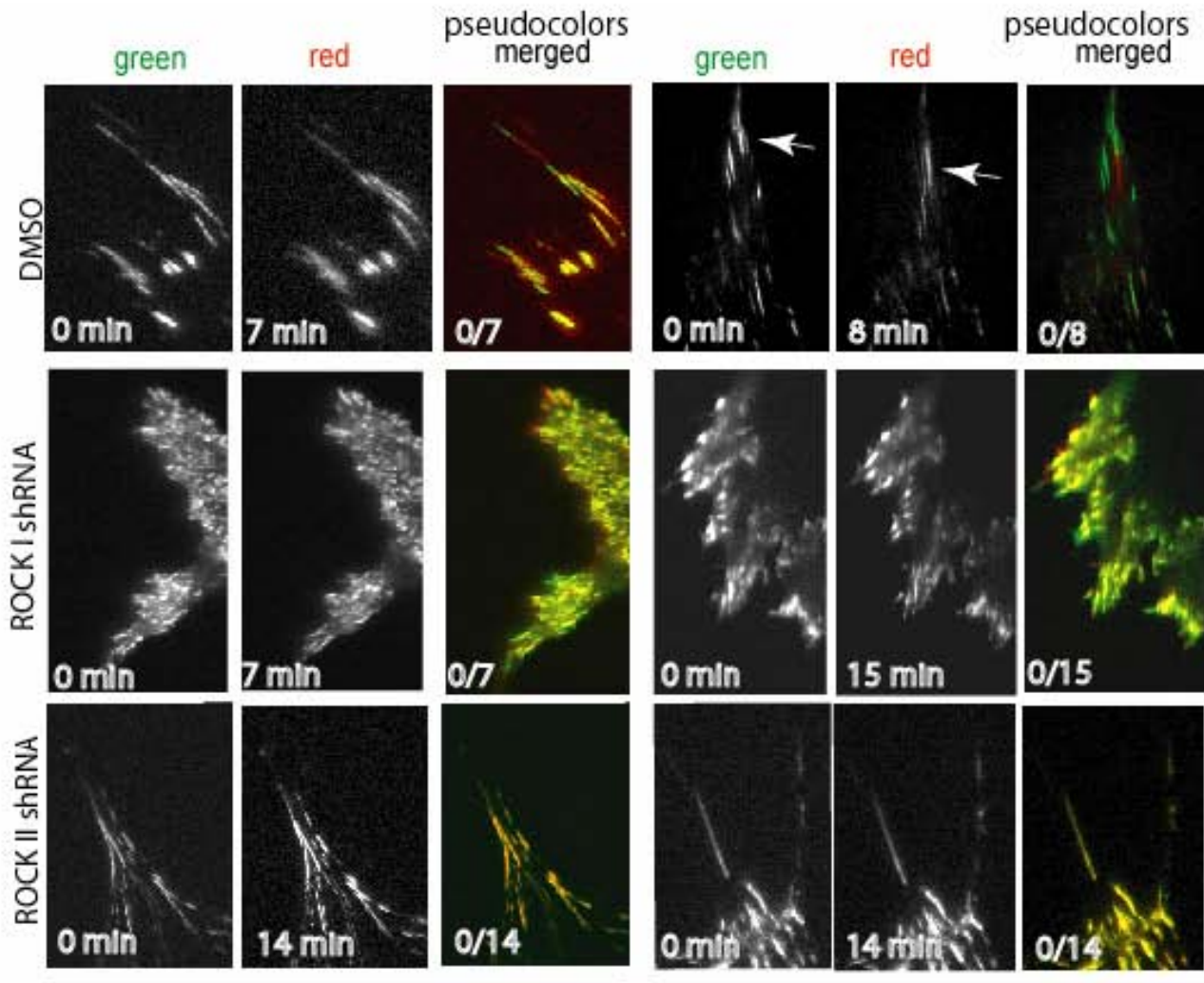

Non Stimulated

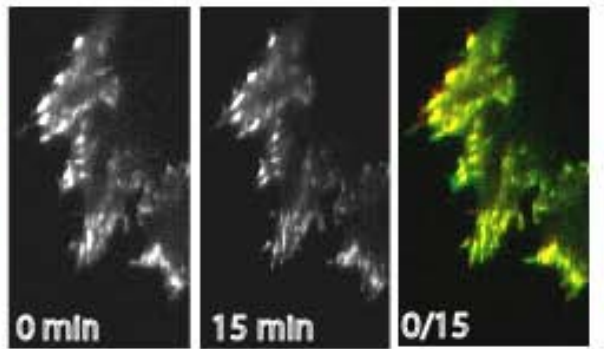

(B)
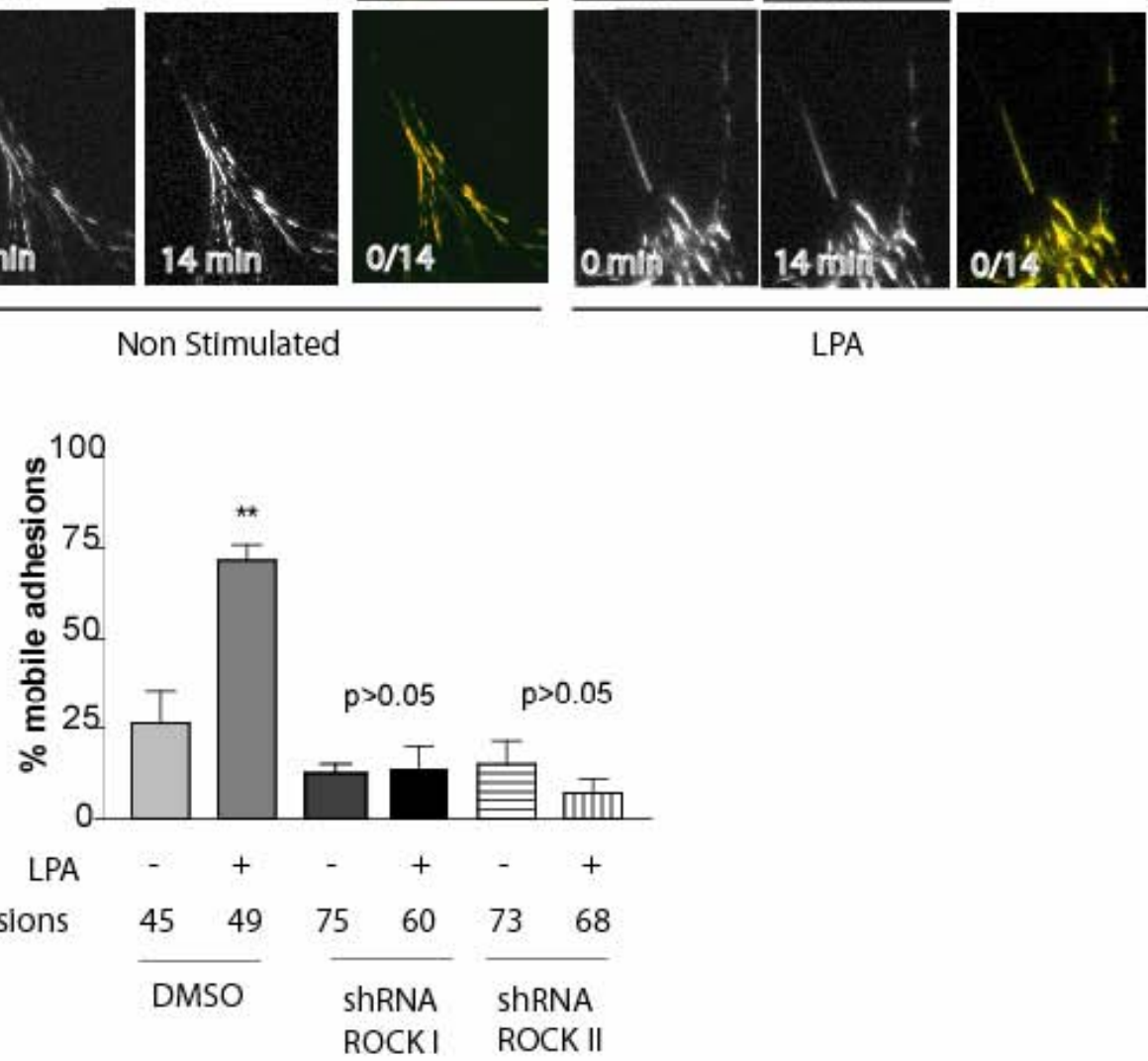
Figure 25. ROCK II is required for LPA induced trailing edge retraction.

Control and ROCK II shRNA treated cells were serum starved for 12 hours. Following the starvation, cells were stimulated with $2 \mu \mathrm{M}$ LPA for 7 hours. Quantification of cellular elongation in LPA stimulated control and ROCK II shRNA treated cells. The experiment was performed 2 times. 
Figure 25

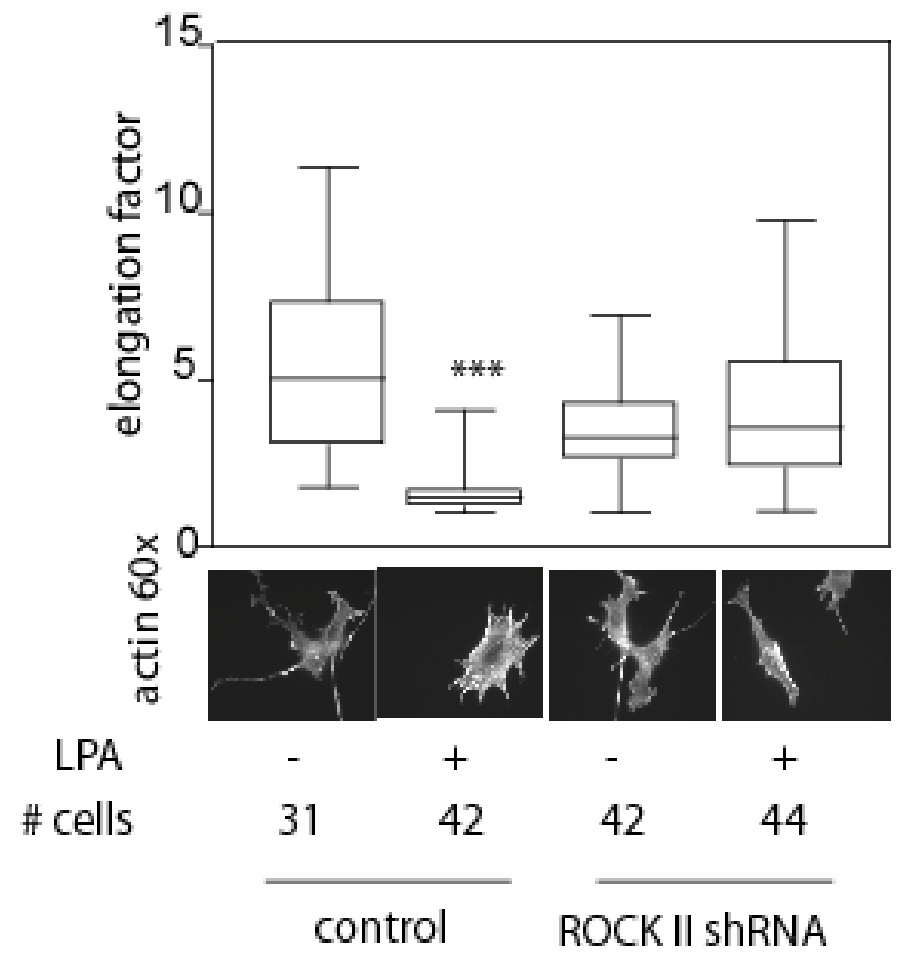


Table 1. Summary of Trailing edge and adhesion movment studies in control, FAK, PDZ-RhoGEF , ROCK I and ROCK II siRNA or shRNA treated cells.

\begin{tabular}{|c|c|c|c|}
\hline sh RNA & $\begin{array}{l}\text { Average } \\
\text { elongation } \\
\text { factor }\end{array}$ & $\begin{array}{c}\text { LPA induced } \\
\text { adhesions movement }\end{array}$ & $\begin{array}{l}\text { LPA induced } \\
\text { trailing edge retraction }\end{array}$ \\
\hline control & 1.2 & YES & YES \\
FAK & 5.2 & NO & NO \\
$\begin{array}{c}\text { PDZ } \\
\text { RhoGEF }\end{array}$ & 4.8 & NO & \\
ROCKI & 1.3 & NO & NO \\
ROCK II & 5.1 & NO & \\
\hline
\end{tabular}

ROCK I shRNA treated NIH3T3 cells are small and do not show any distinct trailing edges. 
Figure 26. Current model of FAK-dependent regulation of adhesion movment and trailing edge retraction.

Activation of LPA receptor results in the activation of the $\alpha$ subunit of G-proteins. PDZRhoGEF that is present on actin filaments via its RGS domain binds to GTP loaded $\alpha$ subunit and catalyzes GTP hydrolysis. FAK/Src, via $\beta 1$ integrin and talin tethered to actin, phosphorylates PDZ-RhoGEF and leads to activation of Rho and Rho effector ROCK II. ROCK II via unknown mechanisms controls adhesion movement and trailing edge retraction. 
Figure 26

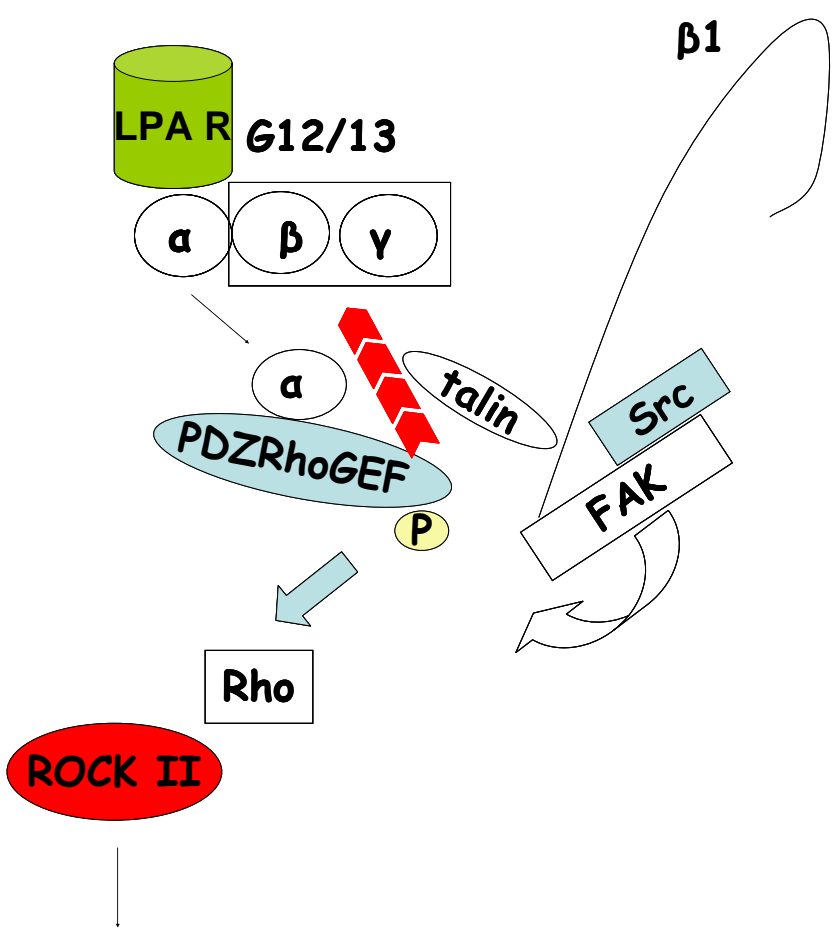

Adhesion movement 


\section{CHAPTER 3}

\section{RACK 1 Organizes FAK-induced ERK Activation and Localization to Adhesions}

\section{Introduction}

ERK signaling converts extracellular stimuli into a variety of specific intracellular biological responses such as differentiation, cell movement, cell division, apoptosis and oncogenic transformation. Extracellular stimuli, including growth factors, hormones and adhesion to ECM proteins, activate Raf, which phosphorylates and activates MEK, which in turn phosphorylates and activates ERK (Chang et al., 2001) (Pearson et al., 2001; Rubinfeld and Seger, 2005). Following activation active ERK localizes to different subcellular compartments including the nucleus, the plasma membrane, endosomes, Golgi apparatus and adhesions (Fincham et al., 2000; Kolch, 2005; Mor and Philips, 2006). At these various locations, ERK can phosphorylate a specific constellation of diverse substrates, leading to changes in gene expression, intracellular trafficking, and cell movement appropriate for the cell and stimulus.

Activated ERK localizes to adhesion structures that through integrins link extracellular matrix proteins to the actin cytoskeleton and anchor the cell to the substratum. Integrin engagement is sufficient to activate ERK, and in cells spreading on fibronectin active ERK localizes to newly formed adhesions (Chen et al., 1994; Fincham et al., 2000). However, the functional significance of active ERK in adhesions has not been well understood.

One mechanism by which ERK signaling can be directed to specific locations and functions is by scaffold or anchor proteins. These non-enzymatic entities associate 
with and enhance functional interaction of the components of the ERK pathway and can regulate the amplitude, timing and location of signals (Kolch, 2005; Morrison and Davis, 2003; Schaeffer and Weber, 1999).

In this work we show that the scaffold protein RACK1 links adhesion signaling to the ERK pathway activation. Furthermore, we demonstrate that RACK1 is required for efficient ERK activation in response to adhesion, but is not necessary for stimulation by growth factor. We also show that RACK1 targets active ERK to a specific intracellular location, adhesion structures found in the protruding areas of the cell, and at this location ERK promotes adhesion disassembly. In addition, we show evidence that FAK is also necessary for ERK activation at adhesion sites. Our data are consistent with the model in which RACK 1 targets the ERK pathway components to adhesions for integrin- and FAK -dependent activation. 
Materials and Methods.

\section{Cell lines and transfections.}

NIH3T3 cells were maintained in Dulbecco's modified Eagle's media (DMEM; Gibco BRL) supplemented with 10\% calf serum (Gibco BRL). CCL39, RAT2 and REF52 cells were maintained in DMEM supplemented with 10\% fetal bovine serum (FBS; Gibco BRL). CCL39 cells were transfected using LipofectAMINE (Gibco BRL) according to manufacturers' instructions. FlipIn system (Invitrogen) was used for generation of NIH3T3 cell line stably expressing GFP-Paxillin. Briefly, NIH3T3 cells were transfected with pFRT/LacZeo vector and a Zeomycin resistant clone (NIH3T3-LacZeo) was isolated. NIH3T3-LacZeo cells were then co-transfected with pOG44 and GFP-Paxillin-pcDNA5FRT constructs, and a Hygromycin resistant cell population (NIH3T3-GFP-Paxillin) was established. NIH3T3-GFP-Paxillin cells have been shown to be GFP-Paxillin positive by western blotting and TIR fluorescence microscopy (data not shown).

\section{Immunoprecipitations and Immunobloting.}

Cells were lysed 24 hours post-transfection in FLAG lysis buffer (50 mM Tris-HCl, 150 $\mathrm{mM} \mathrm{NaCl}, 1 \%$ Triton X-100, 10\% glycerol, 0.5 mM EDTA, 0.5 mM EGTA, pH 7.3 supplemented with $50 \mathrm{mM} \mathrm{NaF}, 5 \mathrm{mM} \mathrm{Na} \mathrm{P}_{2} \mathrm{O}_{7}, 0.2 \mathrm{mM} \mathrm{Na} \mathrm{VO}_{4}$ and protease inhibitors). Clarified extracts were incubated for 2 hours with M2 anti-FLAG affinity resin (Sigma) at $4^{\circ} \mathrm{C}$. Immunoblots were carried out with the following antibodies: antiFLAG M2 (Sigma) and anti-HA 12CA5 (Babcock). Secondary goat anti-rabbit and antimouse antibodies coupled to horseradish peroxidase and enhanced chemiluminescence (ECL) reagents (Amersham Biosciences) were used for protein detection. 


\section{Cell stimulation.}

To determine ERK activation status in response to EGF REF52 cells were cultured in $10 \%$ FBS and either left untreated or serum deprived for 4 hours and stimulated with EGF in DMEM for 10 minutes. To determine ERK activation status in response to adhesion REF52 cells were cultured in 10\% FBS, detached by trypsin, and trypsin was stopped by addition of trypsin inhibitor. Cells were pelleted, resuspended in DMEM, kept in suspension for 90 minutes and plated on dishes precoated with fibronectin $(10 \mu \mathrm{g} / \mathrm{ml})$ for $15 \mathrm{~min}$. Cells were lysed in RIPA lysis buffer and processed as above with the following antibodies: anti-ERK2 B3B9, anti-FAK (Upstate), anti-ppERK, anti-RACK1 and anti-Paxillin (Signal Transduction Laboratories), and anti-FAKpY397. Western blot membranes were developed using ECL (Amersham).

\section{siRNA gene silencing assay.}

A double stranded siRNA targeting the 21-nt sequence AAGGTGTGGAATCTGGCTAAC conserved between the mouse and rat RACK1 genes was synthesized by Qiagen and the Non-Specific Control Duplexes-XIII was obtained from Dharmacon Research. The siRNA was transfected into REF52 and RAT2 cells using the calcium phosphate protocol and 48-72 hours post-transfection cell lysates were probed as indicated. Mock control cells received transfection reagent only. NIH3T3 cells were transfected with Lipofectamine2000 (Invitrogen). A second RACK1 siRNA (GCTAAAGACCAACCACATTTT) was utilized as well and generated identical biological results to those shown in this paper. FAK siRNA oligonucleotide has been described previously (Tilghman et al., 2005). 


\section{Immunostaining.}

Cells were washed twice with PBS, fixed in 1\% paraformaldehyde in PHEM buffer (60 mM Pipes, $25 \mathrm{mM}$ Hepes, $10 \mathrm{mM}$ EGTA, $4 \mathrm{mM} \mathrm{MgSO}$, pH6.9) supplemented with 0.2 $\mathrm{mM}$ vanadate and $50 \mathrm{mM} \beta$-glycerophosphate for $20 \mathrm{~min}$. and extracted with $1 \%$ CHAPS in PHEM buffer for 5 min. Cells were blocked in 20\% normal goat serum (NGS, Invitrogen) in MBST (50 mM MOPS, $150 \mathrm{mM} \mathrm{NaCl}, 0.05 \%$ Tween 20, $\mathrm{pH} 7.4$ ) for $1 \mathrm{~h}$, and incubated with rabbit polyclonal anti-ppERK antibody diluted in 5\% NGS in MBST for $1 \mathrm{~h}$. Cells were washed with MBST three times and subsequently treated with secondary goat anti-rabbit antibody conjugated to biotin (Vector laboratories), diluted in 5\% NGS in MBST for $1 \mathrm{~h}$ followed by incubation with Texas Red-avidin (Vector Laboratories). Paxillin was detected with mouse monoclonal anti-Paxillin antibody and visualized with goat anti mouse antibody conjugated to either FITC (Jackson Laboratories) or Cy5 (Molecular Probes). The following primary antibodies were used: rabbit polyclonal anti-ppERK (diluted 1:50-300; Cell Signaling) and mouse monoclonal anti Paxillin clone 5H11 (1:1000, Upstate). Fluorescent images were acquired using a Nikon Eclipse E600 upright fluorescent microscope equipped with a Hamamatsu ORCA CCD camera. Images were captured and analyzed with OpenLab (Improvision) software.

OpenLab (Improvision) software was used to measure the length of adhesions. For each experimental condition the length (in pixels) of approximately 200 adhesions from 10 cells was measured. Statistical analysis (Mann-Withnay non-paired (not assuming Gaussian distribution) t-test) was done using Prism software (GraphPad Software, Inc). 


\section{TIRF microscopy and adhesion disassembly quantification.}

To record dynamics of adhesions, NIH3T3-GFP-Paxillin stable cell line was grown on Bioptechs T-Dishes (Fisher Scientific) to confluency. To induce cell migration the wound was made and 5 hrs later cells images were acquired every 3 min. Cells were visualized using a Nikon Eclipse TE2000-E inverted microscope equipped with a TIRF illuminator, a 488 argon laser and a 60xDIC TIRF objective (NA 1.45) equipped with a Bioptechs objective heater. Images were acquired with a Retiga 1300i CCD camera and the QCapture Pro software (Q Imaging). To quantify the rates of adhesion assembly and disassembly OpenLab (Improvision) software was used to measure fluorescent intensity of individual adhesions. Changes in fluorescent intensity of adhesion were plotted in a semilogaritmic plot as a function of time, and the constant rate of adhesion disassembly was calculated from the slope as described before. Plotting of individual adhesion disassembly rates and statistical analysis (Mann-Whitney non paired t-test) were done using Prism software (GraphPad Software).

\section{Cell migration assay.}

The bottom sides of 24 well plate cell culture inserts $(8 \mu \mathrm{m}$ pores; Biocoat Becton Dickinson) were precoated with fibronectin $(1.0 \mu \mathrm{g} / \mathrm{ml})$ overnight at $4^{0} \mathrm{C}$. To determine cell migration $1.10^{5}$ REF52 cells were plated on the top of the insert in serum free media and allowed to migrate for 6 hours. Nonmigrating cells were wiped from the top by cotton swab. Cells were washed with PBS, fixed in 3\% paraformaldehyde and stained with crystal violet $(0.1 \%$ in $20 \% \mathrm{EtOH})$. Cell migration was determined by counting the cells at the bottom of five fields. 


\section{Results.}

\section{Identification of RACK1 as a MAPK/ERK pathway scaffold.}

To determine whether RACK1 associates with components of the MAP/ERK Kinase pathway in living cells, we co-expressed RACK1 together with individual components of the ERK pathway and examined their association. RACK1 associated with Raf-1 (Fig. 27A), the ERKs (Fig. 27B) and MEKs (Fig. 27C), supporting the concept that RACK1 can serve a scaffolding function for the ERK pathway.

\section{RACK1 regulates adhesion-induced ERK activation}

RACK1 has been reported to interact with integrin $\beta$ subunits and with the protein kinase Src (Chang et al., 1998). Integrins and Src play important roles in ERK activation during cell adhesion (Chen et al., 1994; Pullikuth et al., 2005; Schlaepfer et al., 1997). We therefore hypothesized that RACK1 may also be important for ERK activation during adhesion. Silencing of RACK1 expression by small interfering RNA (siRNA) in REF52 fibroblasts suppressed ERK activation in cells plated on fibronectin (Fig.28A). RACK1 function was specific to adhesion-induced ERK activation, as RACK1 silencing did not inhibit ERK activation in response to EGF (Fig. 28A). These data are consistent with the hypothesis that scaffold RACK1 localizes MAPK/ERK pathway components for integrin mediated activation. Clearly, growth factor mediated activation of MAPK/ERK pathway must depend on a distinct scaffold from RACK 1. 


\section{RACK1 is required for active ERK localization to adhesions}

Next, we sought to explore the consequences of ERK and RACK1 signaling in response to adhesion. Since RACK1 depletion inhibited ERK activation mediated by integrin engagement to the extracellular matrix, we investigated whether RACK1 controlled the localization of active ERK to adhesions. Control or RACK1-depleted REF52 cells were plated on fibronectin-coated coverslips for $15 \mathrm{~min}$. in serum-free media, fixed, permeabilized under conditions that retain adhesions and immunostained with antibody recognizing diphosphorylated, active ERK and antibody recognizing the adhesion marker paxillin. Consistent with previous reports (Fincham et al., 2000; SlackDavis et al., 2003b) we found that control cells spreading on fibronectin formed peripheral adhesions and a fraction of the cellular pool of active ERK localized to these adhesions (Fig. 28B). RACK1 silencing did not inhibit cell spreading on fibronectin and assembly of adhesions. In fact, RACK1-depleted cells formed visibly more adhesions than control cells (discussed below). Importantly, RACK1 silencing resulted in the absence of active ERK in adhesions (Fig. 28B).

This finding suggests that RACK1 is essential for localization of active ERK to adhesions and that the existing cellular pool of active ERK can not be stably targeted to adhesions in the absence of RACK1. RACK1 silencing also reduced the level of active ERK in adhesions of adherent cells migrating into a wound (Fig. 29) further supporting the idea that active ERK is targeted to newly formed adhesions in a RACK1-dependent process. 


\section{RACK1 promotes adhesion disassembly and cell motility}

In REF52 cells adhering to fibronectin, RACK1 silencing resulted in a noticeable change in adhesion architecture, particularly with respect to adhesion distribution. The cells with silenced RACK1 contained more adhesions, and these adhesions became significantly longer in adherent cells (see Fig. 28B). These observations suggested that regulation of adhesion lifetime could be affected by attenuation of RACK1. Previously published observations suggested that ERK activity is necessary for proper adhesion turnover, particularly adhesion disassembly (Webb et al., 2004). Since ERK presumably needs to act at these adhesions, we hypothesized that RACK1 silencing would specifically alter disassembly of adhesions. To determine whether RACK1 affects the dynamic behavior of adhesions in real time we established a NIH3T3 cell line stably expressing GFP-paxillin and measured the dynamics of adhesions in a wound-healing assay. Adhesions containing GFP-paxillin were recorded using time-lapse total internal reflection fluorescence (TIRF) microscopy, because TIRF microscopy selectively excites fluorophores close to the solid surface (within $\sim 100 \mathrm{~nm}$ ), thus minimizing the background fluorescence of cytoplasmic GFP-paxillin. The wound-healing assay was chosen because cells migrating into the wound display a uniform direction of migration and the adhesions are very dynamic, rapidly appearing and disappearing at the front of the migrating cell. In a wounded NIH3T3 monolayer active ERK localized to dynamic adhesions at the leading edge of the cells, and RACK1 silencing almost completely abolished the appearance of active ERK at the adhesions in the cells migrating into the wound (Fig. 29).

Both control cells and cells with silenced RACK1 migrating into the wound were capable of forming new adhesions at the front of the cell. However, in RACK1-depleted 
cells adhesions at the front of the cell persisted for a longer time than adhesions in control cells (Fig. 30A). The kinetics of adhesion disassembly showed that in the control cells adhesions were disassembled in approximately $10 \mathrm{~min}$ (Fig.30B). By contrast, the disassembly of adhesions in cells with silenced RACK1 was greatly delayed and this defect in adhesion disassembly resulted in enhanced adhesion growth and elongation (Fig. 30A,B). Analysis of the assembly and disassembly rates of dynamic adhesions at the front of the cell showed that RACK1 silencing reduced the disassembly rate by more than $50 \%$ (Fig. 30B) but had no effect on the rate of adhesion assembly (Fig. 30C). These data suggest that RACK1 is a functional component of the ERK signaling pathway that regulates adhesion disassembly.

Since the disassembly of adhesions is an important step in the regulation of cell motility (Webb et al., 2004) we determined whether RACK1 regulates cell motility. In REF52 cells RACK1was depleted by siRNA, and cell migration toward fibronectin was measured in a Boyden chamber assay. Consistent with the reduced adhesion disassembly rate, we found that RACK1 silencing resulted in significantly reduced migration toward fibronectin (Fig. 30D). The reduced rate of adhesion disassembly was also reflected in an increase of adhesion length in cells migrating into a wound (see Fig. 30B). Quantification of the adhesion length showed that in cells plated on fibronectin in the presence of serum, RACK1 knockdown resulted in approximately a two fold increase in adhesion length (Fig. 31A). 


\section{FAK, RACK1 and ERK are components of a signaling pathway that regulates active ERK localization to adhesions and adhesion dynamics.}

RACK1 has been shown previously to associate with Src and regulate its activity (Chang et al., 1998). In addition, Src phosphorylates several adhesion proteins and regulates adhesion disassembly (Parsons, 2003). This raises the question whether the defects in adhesion dynamics seen in RACK1-knockdown cells are a consequence of impaired Src signaling. We thus determined whether pharmacological inhibition of ERK by the MEK inhibitor UO126 affects adhesion length. Similarly to RACK1 knockdown in RAT2 cells ERK inhibition resulted in a significant increase of adhesion length (Fig. 31B). In addition, we observed an increase in adhesion length in cells with silenced ERKs (data not shown). These data are consistent with the previous finding that ERK pharmacological inhibition reduces the rate of adhesion disassembly (Webb et al., 2004).

Our data suggested that integrin engagement is necessary for active ERK localization to adhesions. The activation of Focal Adhesion Kinase (FAK) is a central step in integrin signaling and it has been proposed that both FAK and ERK signaling are linked to adhesion disassembly. These findings indicated that FAK and RACK1 may be components of a signaling pathway that regulates adhesion dynamics through ERK. If FAK regulates adhesion disassembly through ERK it should also regulate presence of active ERK in adhesions. To test this we depleted the FAK protein level by siRNA and determined the localization of active ERK. FAK silencing efficiently decreased the total level of FAK protein (Fig. 32A). Importantly, in spreading cells grown in the presence of serum, FAK silencing completely abrogated the appearance of active ERK in adhesion structures (Fig. 32B). In addition, similarly to RACK1 knockdown we observed that FAK depletion by siRNA resulted in adhesion elongation at the front edge of cells migrating 
into the wound (data not shown). Our data are consistent with the model in which RACK 1 functions in targeting MAPK/ERK pathway to the integrin/FAK pathway for activation to control adhesion dynamics and cell migration.

\section{Discussion.}

In summary, the data presented here show that RACK1 acts as a scaffold for the ERK pathway core kinases and localizes these enzymes to the integrin/FAK adhesion signaling pathway and controls the distribution of active ERK to de novo formed adhesion structures, where it can act on substrates essential for adhesion disassembly and motility. Although RACK1 selectively facilitates ERK activation in response to cellular adhesion, it is dispensable for EGF-mediated ERK activation.

RACK1 associates with multiple components of the ERK pathway including ERK1/2, MEK1/2, and Raf. In addition, RACK1 has been reported to associate with components of the adhesion signaling pathway, including integrin $\beta$-subunits and the Src protein kinase (Chang et al., 1998; Liliental and Chang, 1998), and we show here that it functionally cooperates with FAK. Integrin engagement to ECM potently activates Src (Sandilands et al., 2004), and genetic evidence suggests that Src is an upstream component in integrin mediated ERK activation. In Src deficient cells ERK activation is inhibited in response to adhesion, but the Src family protein kinases are dispensable for ERK activation by the soluble growth factor PDGF (Klinghoffer et al., 1999) (Schlaepfer et al., 1997). Similarly to Src, depletion of RACK1 inhibits ERK activation in response to integrin engagement but not in response to EGF. Thus, the functional and binding characteristics of RACK1 are consistent with its functioning as a scaffold in integrin- 
mediated activation of the ERK pathway and suggest that RACK1 regulates ERK activation by its ability to localize ERK pathway components to adhesions.

Integrin engagement during adhesion not only is sufficient to induce ERK activation but also is required to target active ERK to adhesions. This is shown by the fact that integrin-independent cell adhesion to polylysine neither activates ERK nor targets active ERK to adhesions. Moreover, in cells spreading on fibronectin or in cells with a polarized migratory phenotype, active ERK intensively associates with the presumed sites of acute integrin engagement to extracellular matrix and de novo adhesion formation (Chen et al., 1994; Fincham et al., 2000). Taken together, these data suggest that in migrating cells integrin attachment to the substratum coordinately induces both new adhesion assembly and ERK activation that is spatially linked to these newly formed adhesions. We observed that RACK1 regulates ERK activation specifically in response to integrin engagement to extracellular matrix and that depletion of RACK1 results in the absence of active ERK in adhesions. Importantly, the bulk cellular pool of active ERK is not able to compensate for the absence of active ERK in adhesions when RACK1 is depleted. RACK1 has been reported to localize to adhesions. Our data show that RACK1 regulates adhesion disassembly. Thus, RACK1 may promote ERK pathway activation at the sites of integrin engagement and at the sites of de novo adhesion formation (areas of active protrusions). At adhesion sites ERK signaling facilitates adhesion disassembly consistent with the previous finding that ERK signaling regulates this process. (Webb et al., 2004)

Integrin engagement leads to FAK activation and active FAK localization to de novo formed adhesions. FAK is a central transducer of integrin signaling as it activates numerous pathways including ERK. Previous reports suggested that FAK plays an important role in regulation of adhesion dynamics. It has been shown that FAK null cells 
display an increase in adhesion size and number, probably as a consequence of reduced adhesion disassembly (Ilic et al., 1995; Webb et al., 2004). FAK regulates adhesion turnover at least partly through the ERK pathway because the reduced adhesion disassembly in FAK null cells can be rescued by expression of constitutively active MEK. These data suggest that the ERK pathway acts downstream of FAK to regulate adhesion disassembly.

Since we show that active ERK localization to adhesions is necessary for proper adhesion disassembly these data suggest that FAK may regulate activation of an ERK pool at newly formed adhesions. Indeed, in actively protruding cells we observed that FAK silencing resulted in the absence of active ERK in adhesions. In addition similarly to RACK1 knockdown and ERK pharmacological inhibition, adhesions in FAK silenced cells were significantly longer, suggesting that FAK silencing inhibited adhesion disassembly. Collectively, these data suggest that the tools for adhesion disassembly in protruding areas of the cell are generated coordinately in space and time with the assembly of adhesions, through the ability of RACK1 to bind integrins and the MAP kinase enzymes and to locate these signaling components proximal to the upstream activator FAK.

Expression of constitutively active MEK rescues adhesion disassembly in FAK null cells and, conversely, MEK inhibition decreases the adhesion disassembly rate (Webb et al., 2004). In addition, we observed that MEK inhibition, RACK1 and FAK knockdown result in a similar phenotype, i.e. increase in adhesion length in actively spreading cells. These data suggest that RACK1 regulates adhesion dynamics through the ERK pathway, specifically coupling the ERK pathway to the upstream activator (FAK), consistent with a RACK1 scaffolding function. 
The molecular mechanism(s) by which ERK regulates adhesion disassembly remains to be elucidated. One possibility is that active ERK promotes disassembly of adhesions via suppression of integrin affinity for ECM (Hughes et al., 1997). Myosin light chain kinase (MLCK) is a potential downstream target, as it has been shown to be phosphorylated by ERK and to regulate adhesion disassembly (Klemke et al., 1997). ERK may also activate proteases such as calpain to induce calpain-mediated proteolysis of adhesion components and thus regulate adhesion disassembly (Carragher et al., 2003; Franco et al., 2004; Glading et al., 2004). In addition, ERK phosphorylates paxillin on serine residue 83 which may modulate the association of specific adhesion molecules (Ishibe et al., 2004). In summary, we propose that RACK1 serves as a scaffold protein that organizes integrin- and FAK- induced ERK activation and localization to adhesions. In this way, a pool of active ERK is created that is spatially and temporally restricted to the site of integrin engagement and adhesion formation and is separated from bulk cellular ERK signaling, providing a specific channel for activation and regulation of the MAP Kinase pathway. This localized ERK activation, which is generated coordinately with integrin-induced adhesion assembly, represents a negative regulatory loop which promotes adhesion disassembly. 


\section{Figures}

Figure 27. RACK1 associates with components of ERK pathway.

(A) Co-immunoprecipitation of Raf-1 with RACK1. CCL39 cells were transfected with either control vector or FLAG-RACK1 and untagged Raf-1. RACK1 precipitation and immunodetection were performed as in Fig. 1A with the exception that Raf-1 antibody was used to detect Raf-1. (B) Co-immunoprecipitation of RACK1 with ERK1 and ERK2. CCL39 cells were transfected with control vector, FLAG-ERK1 or FLAG-ERK2 constructs and HA-RACK1. ERK precipitation and immunodetection were performed as

in Fig. 1A and RACK1 was detected with HA antibody. (C) Co-immunoprecipitation of RACK1 with MEK1 and MEK2. CCL39 cells were transfected with either control vector, FLAG-MEK1 or FLAG-MEK2 constructs and HA-RACK1. MEK precipitation and immunodetection were performed as in Fig. 1B. Upper band in MEK blot represents FLAG antibody heavy chain)

\section{Experiment performed by Tomas Vomastek}


Figure 27.

A.

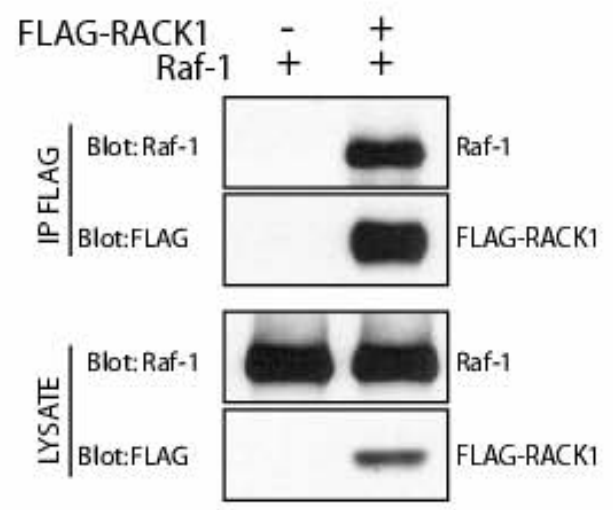

B.
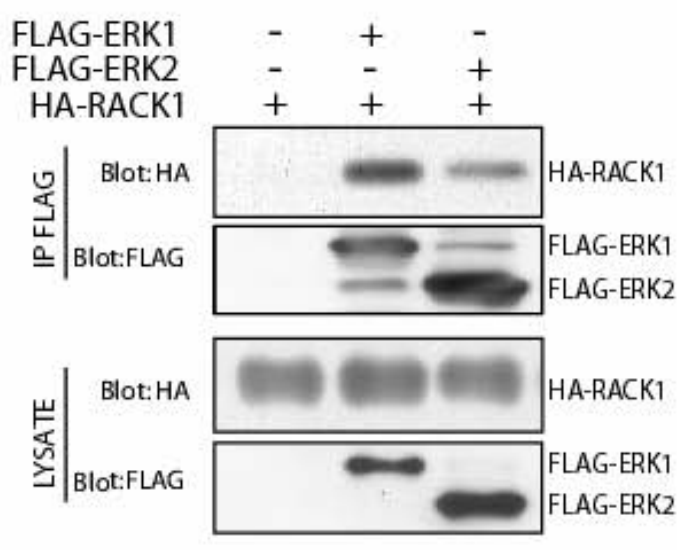

C.

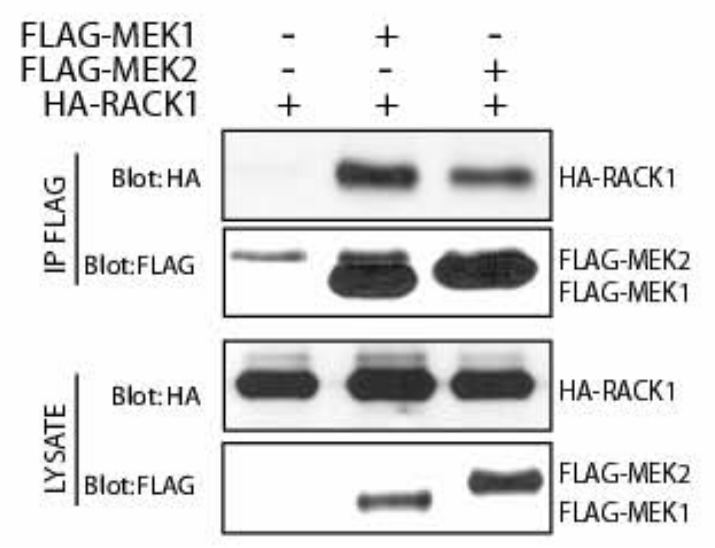


Figure 28. Suppression of RACK1 interferes with ERK activation in response to adhesion.

(A) Downregulation of endogenous RACK1 protein inhibits ERK activation in response to adhesion. REF52 cells were transfected with siRNA for 48 hours, suspended in serumfree medium for 90 minutes and replated on fibronectin coated dishes for 15 minutes. Cell lysates were probed with antibody recognizing doubly phosphorylated active form of ERK and with antibody directed against ERK2 and RACK1 to confirm equal loading of proteins and knockdown efficiency, respectively. Depletion of RACK1 protein by siRNA targeting different regions of RACK1 mRNA also inhibited ERK activation in response to adhesion to fibronectin confirming the siRNA specificity (data not shown). (B) RACK1 silencing inhibits active ERK localization to substrate adhesions in response to fibronectin. REF52 cells were transfected with siRNA for 48 hours, suspended and replated on fibronectin for 15 minutes. Cells were co-stained with antibodies recognizing active form of ERK (red) and paxillin (green). The inset shows a higher magnification of boxed area. Cell lysates were probed with antibody directed against RACK1and ERK2 to confirm knockdown efficiency and equal loading of proteins, respectively (right panel)

Experiment performed by Tomas Vomastek (A) and Marcin Iwanicki (B) 
Figure 28

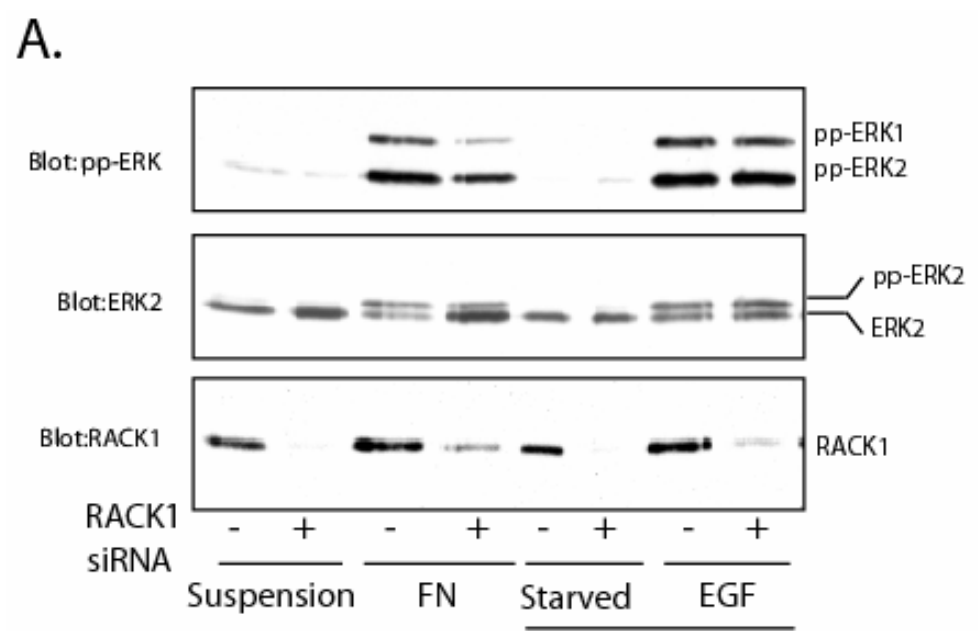

B.

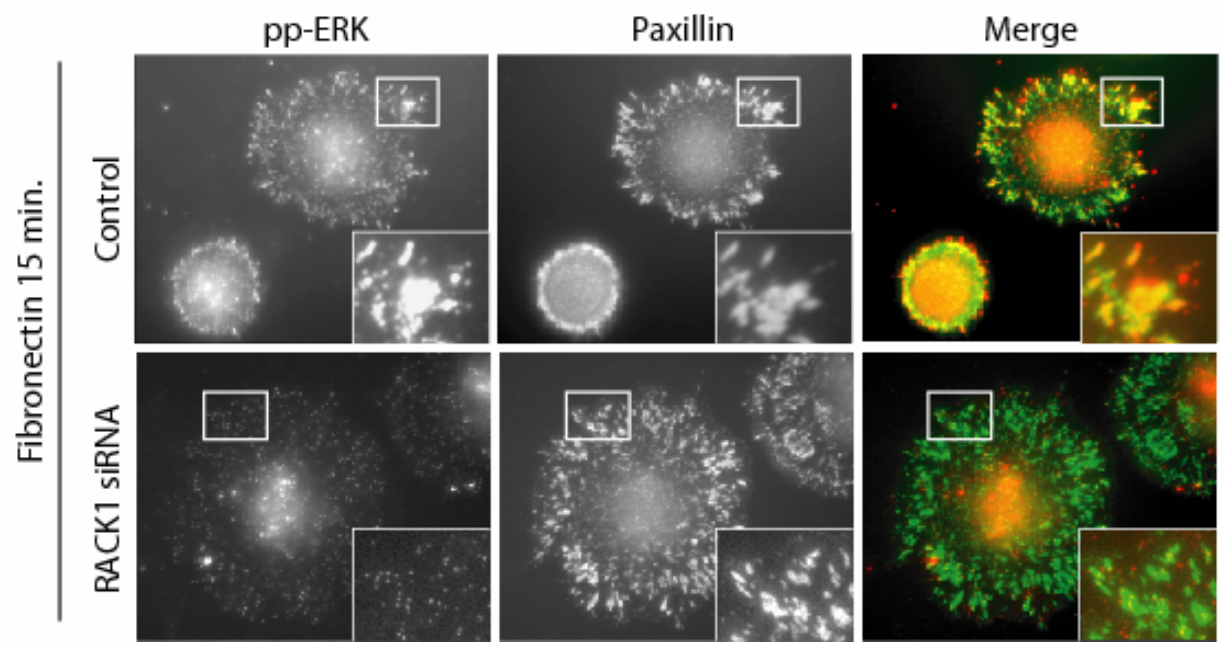


Figure 29. RACK 1 regulates the localization of active ERK to adhesions in wounded cell monolayers.

NIH3T3 cells were transfected with siRNA and 48 hours after the transfection wound was made in confluent monolayer of cells in the presence of $10 \%$ serum. 5 hours after the wound cells were co-stained with antibodies recognizing the active form of ERK (red) and paxillin (green). The inset shows a higher magnification of boxed area. Western blot shows levels of RACK 1 expression in this experiment. Experiment performed by Marcin Iwanicki. 
Figure 29
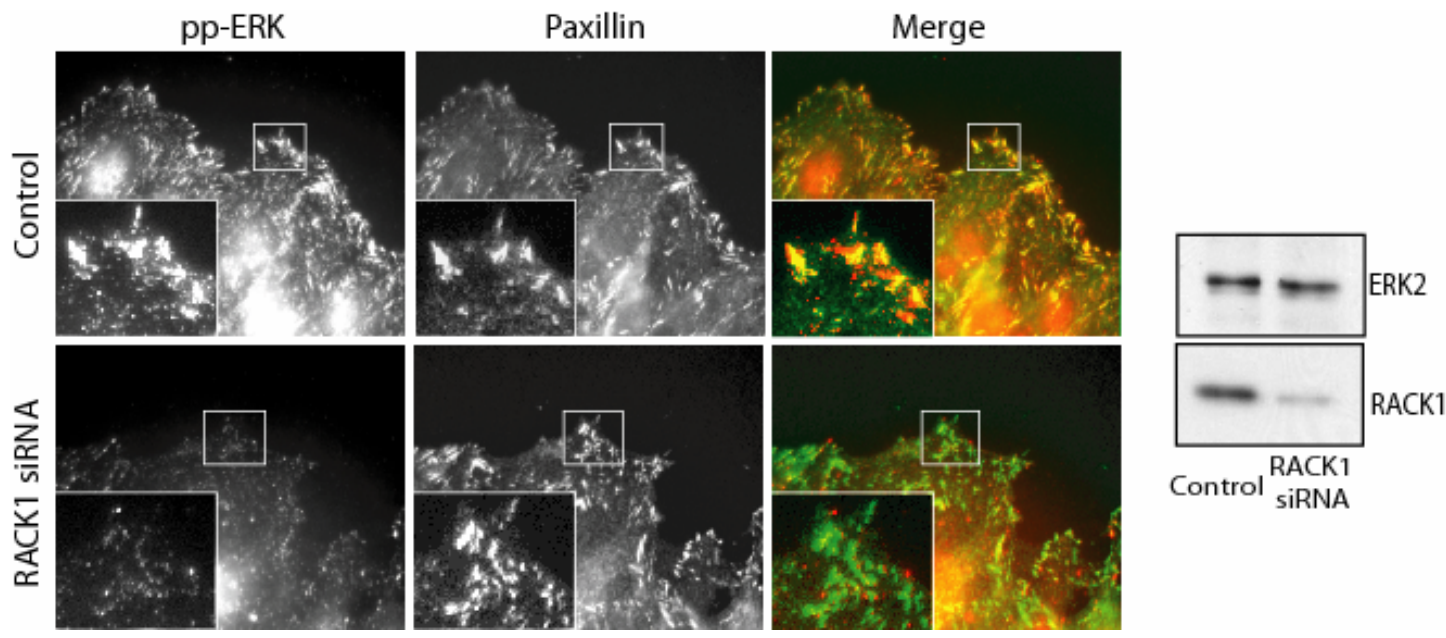
Figure 30. RACK1 regulates adhesion disassembly and cell motility.

(A) Series of images from time-lapse TIRF microscopy recording the dynamics of GFPpaxillin adhesions in control (upper panel) and RACK1 knockdown (lower panel) cells. Arrows indicate examples of dynamic adhesions at the protrusive region of the cell where both assembly and disassembly were seen. NIH3T3 cells stably expressing GFP-paxillin were transfected and wounded as described in Fig. 22. GFP-paxillin containing adhesions were imaged 5 hrs after wounding by time-lapse TIRF microscopy by collecting images every 3 min. (B) Rate of adhesions disassembly. Rate constants of adhesion disassembly were determined from values obtained from 37 adhesions in 4 cells (control cells) or 30 adhesions in 5 cells (RACK1 siRNA treated cells). Each point represents one adhesion analyzed. Only adhesions that displayed an initial increase in fluorescent intensity (i.e. adhesion assembly) were included in the analysis. (C) Rate of adhesion assembly. Rate constants of adhesion assembly were determined from values obtained from 16 adhesions in 4 cells (control cells) or 15 adhesions in 5 cells (RACK1 siRNA treated cells). (D) RACK1 depletion inhibits cell migration toward fibronectin. REF52 cells were transfected with siRNA for 48 hours, and suspended, and cell migration toward the fibronectin was measured in triplicates in a Boyden chamber assay. The data represent the average +/- standard deviation. Cell lysates were probed with antibody directed against RACK1and ERK2 to confirm knockdown efficiency and equal loading of proteins, respectively (right panel). Depletion of RACK1 protein by siRNA targeting different regions of RACK1 mRNA also inhibited cell migration toward fibronectin (data not shown). Experiment performed by Marcin Iwanicki (Rodriguez-Frade et al.) and Tomas Vomastek (D) 
Figure 30

A.
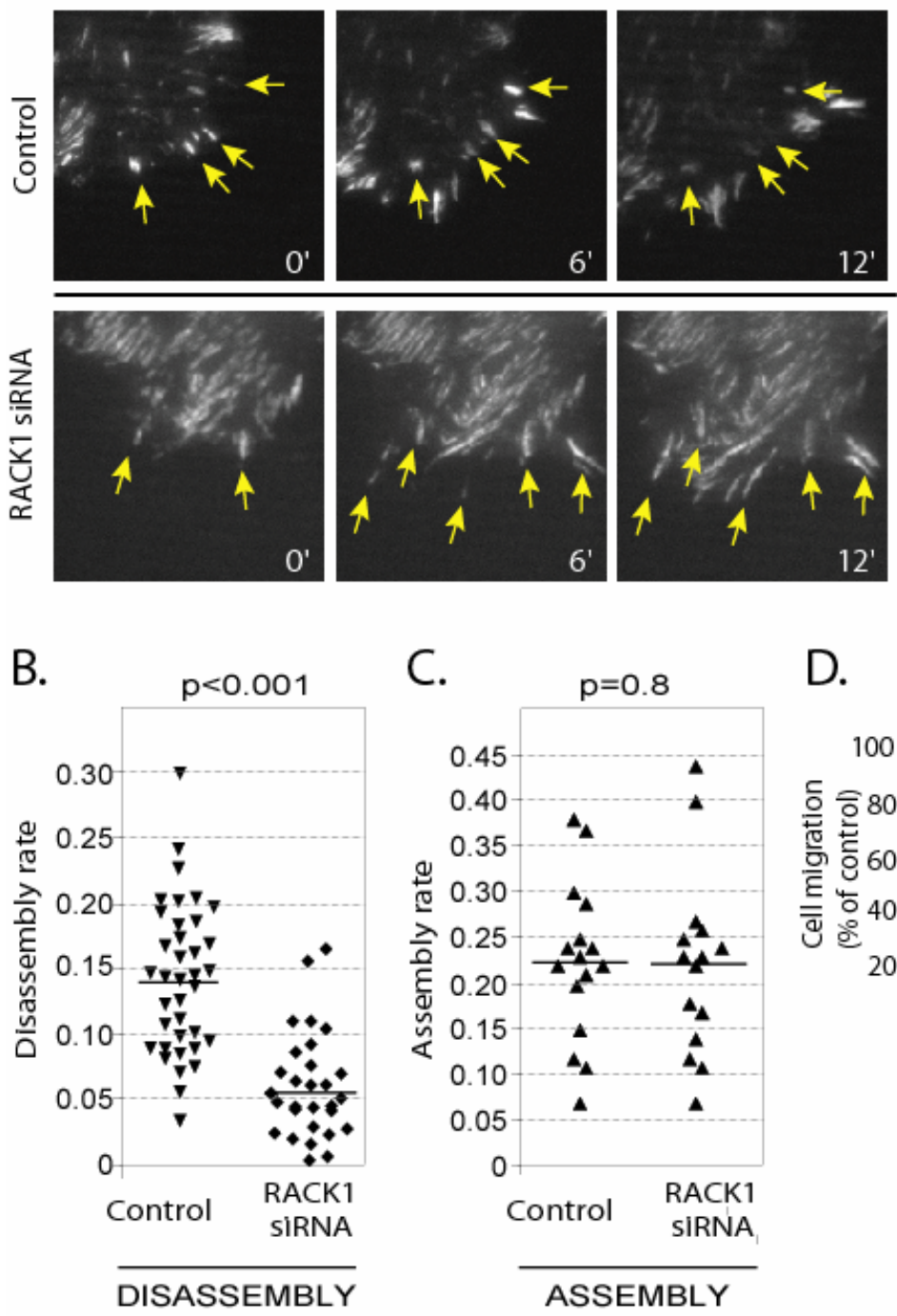

D.
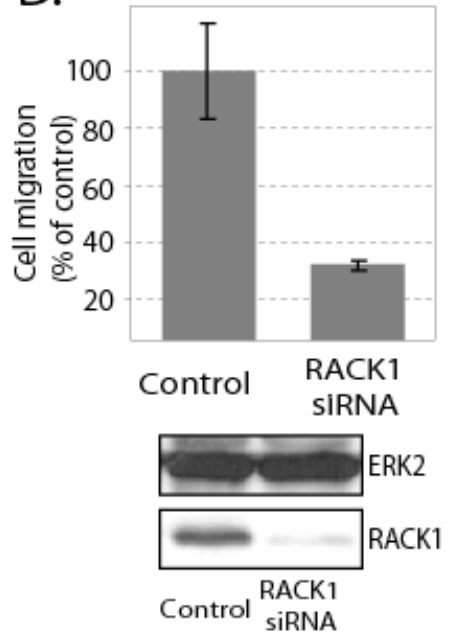
Figure 31 RACK1 and ERK regulate adhesion length.

(A) RACK1 silencing increases adhesion length. RAT2 cells were transfected with siRNA, and 48 hours after the transfection cells were plated on fibronection $(1 \mathrm{ug} / \mathrm{ml})$ coated coverslips in the presence of $10 \%$ serum. 4 hours after the plating cells were stained with antibody recognizing paxillin. Right panel shows the quantification of adhesion length in control and RACK1 siRNA transfected cells (detrmined from values obtained from $\sim 200$ adhesions in 10 cells). Only peripheral adhesions were included in the analysis. Depletion of RACK1 protein by siRNA targeting different regions of RACK1 mRNA also increased the length of the adhesions (data not shown). (B) MEK inhibition induces increase in adhesion length. RAT2 cells were plated on fibronectin $(1 \mu \mathrm{g} / \mathrm{ml})$ coated coverslips and treated with the MEK inhibitor UO126 $(10 \mathrm{mM})$ for 48 hrs. Cell staining and quantification of adhesion length were done as in A. Experiment performed by Marcin Iwanicki (A) and Tomas Vomastek (B). 
Figure 31

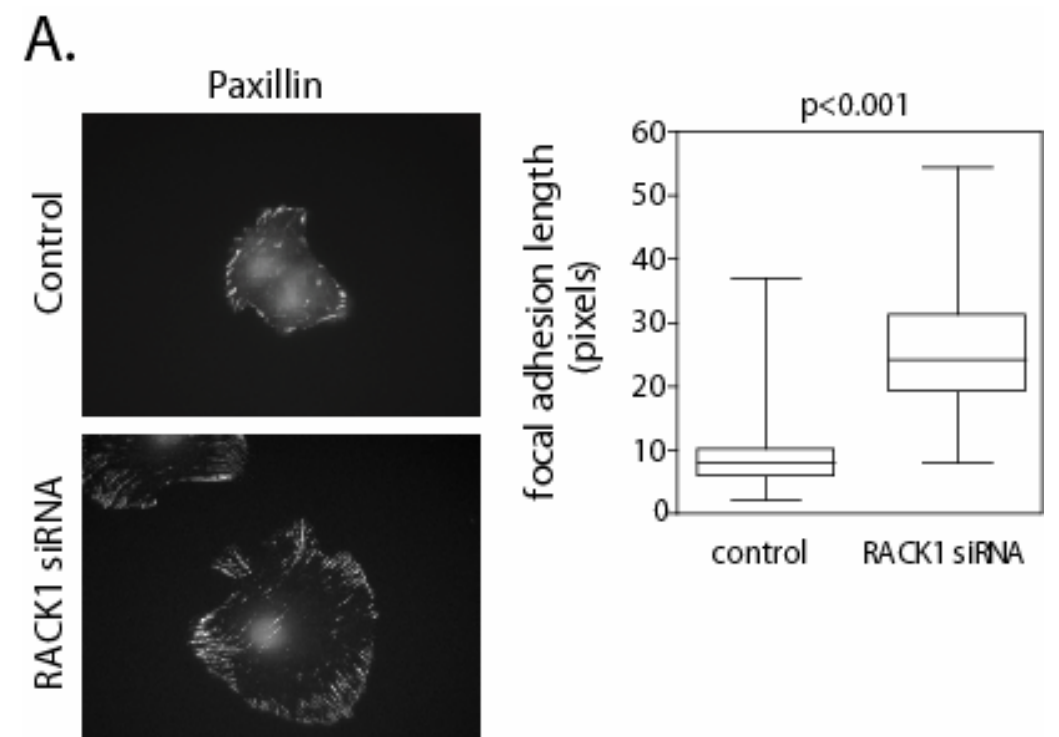

B.
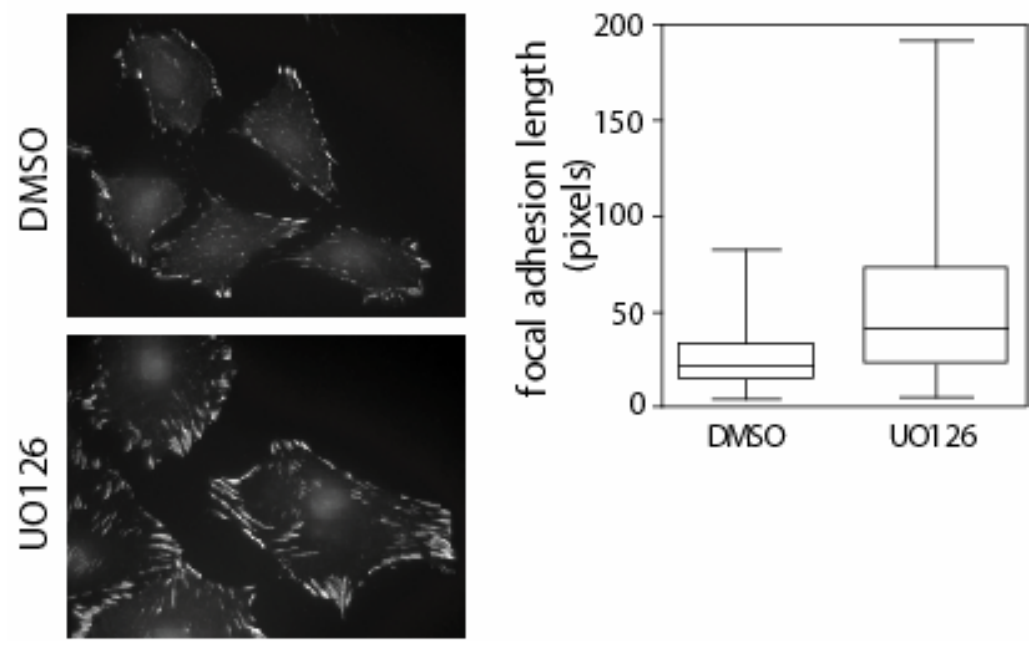


\section{Figure 32. FAK regulates active ERK localization to adhesions} and adhesion length .

(A) Efficiency of FAK knockdown. RAT2 cells were transfected with FAK siRNA for 48 hours, suspended in medium with $10 \%$ serum and replated on fibronectin-coated dishes for 4 hours. Cell lysates were probed with antibodies recognizing FAK, active FAK (FAKpY397), ERK and active ERK. (B) FAK silencing inhibits active ERK localization to adhesions. RAT2 cells were transfected with FAK siRNA for 48 hours, suspended in medium with $10 \%$ serum and replated on fibronectin coated coverslips. 4 hours after the plating cells were co-stained with antibodies recognizing the active form of ERK (red) and paxillin (green). Graph represents quantification of adhesion length in control and FAK siRNA treated cells.

\section{Experiment performed by Tomas Vomastek.}


Figure 32

A.

SiRNA 志

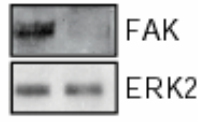

B.

Paxillin

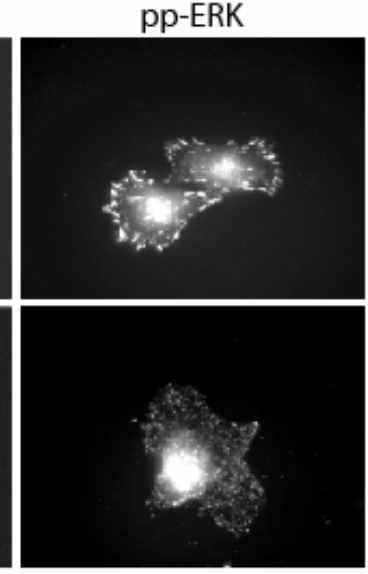

Merge
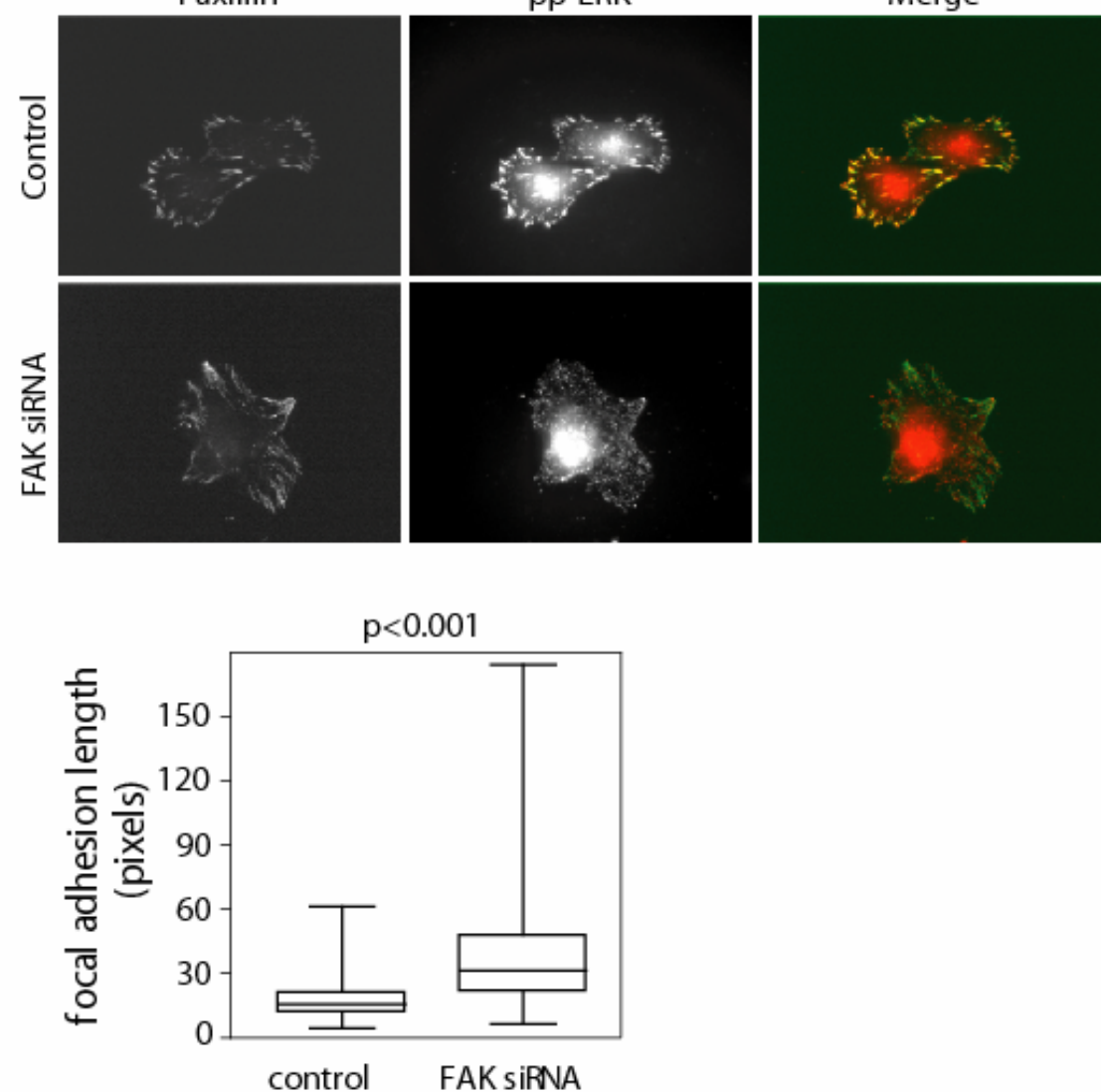


\section{CHAPTER 4}

\section{Summary and possible models}

Cell migration is a biological process required for development and adult tissue homeostasis. The mechanical events that characterize cell migration include polarized protrusion of the lamellipodium and distinct trailing edge retraction. Cells that migrate form and disassemble adhesion structures within the protruding lamellipodia and mature, move and break adhesions within the trailing edges. The goal of this research was to identify the signaling pathways that control dynamic behavior of adhesions found in the rear and the front of polarized fibroblasts.

In chapter 2 of this dissertation, we described experiments that showed that FAK, through the involvement of PDZ domain-containing guanine nucleotide exchange factor, couples Rho and ROCK II signaling at mature adhesions to control adhesion movement (treadmilling) and trailing edge retraction. In chapter 3 of this dissertation, we identified the requirements for the scaffold protein RACK 1 in localizing the ERK pathway components to integrin/FAK signaling to control adhesion disassembly in the protruding lamellipodia of fibroblasts.

In the first and second part of this discussion, I will summarize the data and present major conclusions drawn thus far. In the third part of the discussion, I will combine conclusions from part 1 and part 2 and present a model for signal-dependent spatial regulation of adhesion dynamics during cell migration. 


\section{Role of FAK in fibroblast trailing edge retraction and adhesion movement.}

First insights into the role of FAK in cell migration came from the studies using fibroblasts that were derived from embryos in which the FAK gene had been disrupted. FAK-null fibroblasts plated on fibronectin-coated dishes showed two-fold reduced migration as compared to control cells. In addition, in steady state growth conditions, FAK null fibroblasts had enlarged adhesions (Ilic et al., 1995). These data suggested that the events that regulate adhesion life cycle could be directly linked to the migratory process and FAK was part of this process. Later, two studies showed that FAK null fibroblasts did not properly regulate Rho activity in response to fibronectin adhesion (Ren et al., 2000) and LPA stimulation (Palazzo et al., 2004). These studies indicate that FAK participates in RhoA mediated regulation of adhesion and cytoskeleton dynamics. Recent studies showed that the FAK-Src complex phosphorylated certain Rho GEFs and promoted Rho activation (Chikumi et al., 2002; Zhai et al., 2003) further arguing for a role for FAK in Rho regualtion.

In chapter 2 we provide the first evidence that FAK signaling to a specific adhesion/actin associated Rho guanine nucleotide exchange factor regulates Rho GTPase-dependent dynamics of mature adhesions found in trailing edges of polarized fibroblasts. The siRNA mediated attenuation of FAK expression in fibroblastic cells resulted in stabilization of trailing edges and overall cellular elongation, suggesting defects in trailing edge retraction. Similar phenotypes were observed in serum starved fibroblasts, suggesting that trailing edge retraction is driven by a factor present in the serum. In serum-starved fibroblasts, activation of LPA receptors that are coupled to heterotrimeric G proteins but not activation of tyrosine kinase receptors (e.g PDGFR) resulted in rapid trailing edge retraction and restoration of normal cell morphology. These 
data indicate that trailing edge retraction was regulated by events downstream of heterotrimeric G proteins. LPA-induced trailing edge retraction was accompanied by the activation of FAK and rapid inward movement of GFP-paxillin- or GFP-FAK- containing adhesions found in the trailing edge of fibroblasts. Furthermore, siRNA-mediated attenuation of FAK expression in fibroblasts blocked LPA receptor-mediated trailing edge retraction and inward movement of GFP-paxillin-containing adhesions. These data indicate that FAK is a key component, regulating trailing edge retraction and adhesion movement in response to activated $\mathrm{G}$ proteins.

It is well established that activation of LPA receptors ( $G$ protein-coupled receptors) increases tyrosine phosphorylation of FAK (Luttrell et al., 1997) and induces Rho mediated cytoskeletal changes (Ridley and Hall, 1992). LPA-mediated activation of Rho is very well understood (Moolenaar et al., 2004). Binding of LPA to LPA receptors induces association of LPA receptors with heterotrimeric $\mathrm{G}$ proteins (G12, G13). The alpha subunit of the heterotrimer binds GTP and upon interaction with the RGS domaincontaining Rho GEF becomes activated. Following this interaction, Rho GEF binds via its DH domain to Rho GTPase and facilitates exchange of GDP to GTP (Fukuhara et al., 1999; Reuther et al., 2001; Togashi et al., 2000; Zheng et al., 1995a). As shown in Fig. 20, we find that FAK forms a complex and co-localizes with the RGS domain-containing Rho GEF (PDZ-RhoGEF). In addition, ectopic expression of PDZ-RhoGEF but not PDZRhoGEF lacking the DH domain or p115RhoGEF (a close family member that does not localize to adhesions) rescued trailing edge retraction in cells where FAK was attenuated. These data argue that this downstream effector of G12/G13 can rescue trailing edge retraction in the absence of FAK.

Consistent with these results, we observed that siRNA-mediated inhibition of PDZ-RhoGEF in fibroblasts resulted in the phenotype that was similar to that observed in 
FAK siRNA treated cells. PDZ-RhoGEF siRNA treated cells exhibited an elongated phenotype in the presence of $10 \%$ serum, indicating defects in trailing edge retraction. Indeed, LPA stimulation of adhesion dynamics or trailing edge retraction in PDZRhoGEF siRNA treated cells was significantly decreased. These observations are in agreement with the previous published results showing that the FAK/Src complex phosphorylates PDZ-RhoGEF and promotes Rho activation in response to G12/G13 agonists (Chikumi et al., 2002).

FAK has been shown to regulate Rho activity through promoting phosphorylation of a different RhoGEF, p190-RhoGEF (Zhai et al., 2003). In contrast to PDZ-RhoGEF, p190RhoGEF does not have an RGS domain and it has not been shown to be downstream effector of heterotrimeric G proteins (Rossman et al., 2005). However, similar to PDZ-RhoGEF, p190 RhoGEF localizes to adhesions and siRNA-mediated attenuation of p190 RhoGEF in fibroblasts induces cell elongation, suggesting a defect in trailing edge retraction (these data were published concurrently with our data) (Lim et al., 2008). Both observations are consistent with the model in which FAK-dependent localized activation of Rho induces adhesion inward movement that is concomitant with trailing edge retraction.

Fibroblast trailing edge retraction depends on actomyosin regulation (Riento and Ridley, 2003; Vicente-Manzanares et al., 2007). ROCK has been shown to directly regulate actomyosin contractility through modulation of myosin light chain phosphorylation (Yoneda et al., 2005). In our experiments, restoration of trailing edge retraction in FAK siRNA-treated cells depended on Rho-mediated activation of ROCK I/II but not the other Rho A effectors, mDia I /II. Consistent with these data, we showed that siRNA-mediated attenuation of ROCK II but not ROCK I resulted in a similar phenotype as FAK or PDZ-RhoGEF knock-down cells. ROCK II- but not ROCK I- 
depleted cells became elongated, with prominent mature adhesions in the trailing edge. LPA-induced trailing edge retraction and adhesion movement are dependent on ROCKII. Interestingly, cells in which expression level of ROCK I was decreased did not become elongated and had very small adhesions, a phenotype opposite to FAK, PDZ-RhoGEF and ROCK II knock-down cells. This observation is consistent with the requirement for ROCK I to maintain actomysoin contractility and adhesion stability.

These data indicate that ROCK I and ROCK II are not redundant and contribute differently to trailing edge retraction and adhesion dynamics in fibroblasts. Recently published studies support the differential roles of ROCK I and ROCK II in the regulation of actomyosin and adhesion (Samarin et al., 2007; Yoneda et al., 2005; Yoneda et al., 2007). In these studies, ROCK II attenuation did not alter myosin activation as evidenced by MLC phosphorylation, actomyosin or adhesion integrity, the events sensitive to ROCK I depletion. Our studies also showed that ROCK II, FAK or PDZ-RhoGEF knockdown cells did not exhibit alterations in LPA-induced MLC phosphorylation (data not shown). In preliminary experiments, shRNA-mediated attenuation of FAK, ROCK II and PDZ-RhoGEF, increased adhesion size, whereas attenuation of ROCK I decreased adhesion size, indicating that ROCK I regulates adhesion dynamics distinctly from FAK, PDZ-RhoGEF and ROCK II. One possibility is that ROCK I directly regulates via increased actomyosin contractility adhesion assembly and maturation. ROCK I signaling to the actomyosin may be triggered by the activation of the LPA receptor and downstream signals independently of FAK, PDZ-RhoGEF and ROCK II. FAK, PDZRhoGEF and ROCK II however, may play a role in promoting adhesion disassembly. Therefore, lack of adhesion movement (e.g., treadmilling) in the absence of FAK, PDZRhoGEF or ROCK II may reflect the failure to stimulate the turnover of components within the adhesion. Since adhesions remain under tension from the ROCK I pathway, 
addition of new molecules to the adhesions could take place and be independent of the FAK/PDZ-RhoGEF/ROCK II pathway. In support of this hypothesis, we have observed in preliminary experiments that adhesions increase in length in cells lacking the expression of FAK, PDZ-RhoGEF or ROCK II.

The mechanisms that regulate adhesion treadmilling are poorly understood. It is clear that adhesion treadmilling is contractility-dependent adhesion assembly at the one end of the adhesion and dissasembly at the other end of the adhesions. We speculate that the LPA/FAK-dependent RhoA and ROCK II activation contributes to adhesion disassembly and that LPA/RhoA/ROCK I activation increase actomyosin contractility and promotes adhesion assembly. Therefore, the ROCK I and the ROCK II pathways are both necessary for adhesion treadmilling.

\section{Role of RACK 1 in the regulation of FAK /ERK-dependent adhesion disassembly in the lamellipodia of migrating cells.}

A key event in directional migration is the formation of protrusive lamellipodia in the direction of cell migration. In the lamellipodia of polarized fibroblasts, adhesion dynamics are tightly controlled by temporal and spatial organization of signaling modules within adhesions. In Chapter 3 of this thesis, we presented evidence that the integrinlinked scaffold protein RACK 1 associates with core components of the MAP kinase pathway and targets this pathway to adhesions for activation.

A plethora of receptor agonists will activate the MAP kinase pathway (Kolch, 2005). The mechanism by which ERK is coupled to different receptors depends on scaffold or anchoring proteins (Kolch, 2005). These non-enzymatic molecules channel the ERK pathway to upstream activators. For instance, MAP Kinase Partner 1 (MP1) 
associates with MEK and ERK and present this pathway to the upstream activator Rac/cdc42-PAK pathway (Pullikuth et al., 2005). MP1, by virtue of its ability to interact with MEK 1 and PAK, regulates phosphorylation of serine residue 298 of MEK 1 and primes MEK for activation by Raf (Pullikuth et al., 2005).

Consistent with these results, we observed that another scaffold protein RACK 1 tethers components of the MAP kinase pathway (Raf, MEK and ERK) and presents this pathway to upstream activators. A role for PAK in the activation of this pathway is indicated by our observation that in the absence of RACK 1, integrin-dependent activation of Raf by PAK (data not shown) and subsequent ERK activation was decreased. These results lead us to propose that RACK 1 is a scaffold that links integrin engagement with PAK/Raf-dependent MAP kinase activation. RACK 1 binds MP1 a scaffold protein for MEK, ERK and PAK, thus, it is possible that in the absence of RACK 1, PAK is never recruited to the complex (Pullikuth et al., 2005) and this results in the failure to activate the ERK pathway (Figure 33).

Integrin engagement results in the formation of distinct adhesion structures. Activated ERK has been shown previously to be part of adhesions in cells spreading on fibronectin (Slack-Davis et al., 2003a). Consistent with these data, we observed that attenuation of RACK 1 expression by siRNA altered localization of active ERK in adhesions. In cells with actively protruding lamellipodia, RACK 1 was required for activated ERK localization to de novo formed adhesions. Our data are consistent with a model in which RACK 1 targets components of MAP kinase signaling to the integrin pathway.

One of the properties of MAP kinase signaling is the ability to regulate adhesion disassembly and cell migration (Webb et al., 2004). Cells treated with MEK inhibitor migrate poorly and have low rates of adhesion disassembly. Consistent with these data, 
we observed that siRNA attenuation of RACK1 expression impaired cell migration and adhesion disassembly in the protruding areas of the cell. A reduced rate of adhesion disassembly during wound-induced migration was also reflected in an increase of adhesion length, suggesting reduced turnover of these adhesions. Our data argue that coupling of the ERK pathway to newly assembled adhesions within lamellipodia provides a negative feedback loop that promotes adhesion disassembly.

The disassembly rates of adhesions in the lamellipodia depend on FAK signaling. Forced expression of activated MEK in FAK null cells partially rescues adhesion disassembly rates in cells with protruding lamellipodias (Webb et al., 2004). Furthermore, FAK has been shown to regulate integrin-dependent activation of MAPK during acute integrin engagement (Slack-Davis et al., 2003a). Consistent with these data, we observed that FAK was necessary for active ERK localization to adhesions in the presence of RACK 1. Furthermore, similar to RACK 1-depleted cells or MEK inhibitortreated cells, FAK siRNA treated cells showed elongated focal adhesions. These results agree with the model in which RACK1 organizes MAP kinase core components and recruits it to adhesions for the activation.

The biochemical mechanisms by which ERK contributes to regulation of adhesion disassembly remain to be elucidated. Studies focusing on identification of new substrates for ERK during acute integrin engagement are likely to shed some light. Nevertheless, ERK has been shown to phosphorylate substrates implicated in the regulation of adhesions (Klemke et al., 1997), (Ishibe et al., 2004) (Carragher et al., 2003; Franco et al., 2004) and to inhibit activation of integrins (Hughes et al., 1997), which most likely leads to weakening of adhesions and adhesion dissasembly. However, in all these examples, the requirement for ERK specific phosphorylation in the regulation of adhesion dynamics is yet to be determined. 


\section{FAK couples two distinct signaling pathways to spatially regulate adhesion dynamics.}

Protrusions at the front of the cell and trailing edge retraction are tightly coupled to the regulation of substrate adhesions found in the front and back of migrating fibroblasts (Vicente-Manzanares et al., 2007). In the protruding areas, adhesions are stationary but disassemble at relatively fast rates. On the other hand, during trailing edge retraction, adhesions move in the inward direction but disassemble slower than front adhesions (Palecek et al., 1998; Smilenov et al., 1999). The work presented in this thesis argues that the spatial dichotomy of adhesion behavior is regulated by two distinct signaling pathways with a common initiator. Based on our studies we suggest the following model.

During lamellipodial adhesion formation, integrin activation leads to adhesionlocalized activation of the ERK pathway. In this model, integrin-dependent activation of ERK relies on two events: First, the integrin-linked scaffold RACK1 recruits MAP kinase core components to the adhesion. Second, activation of FAK results in the downstream activation of MAP kinase components via one or more pathways (Figure 33). Therefore, FAK-dependent activation of MAP kinase at de novo formed adhesion sites primes these adhesions for disassembly. FAK-mediated activation of the ERK pathway may occur via different routes. First, phosphorylation of FAK by Src at tyrosine 925 may initiate the Grb2/Ras pathway leading to activation of the Raf kinase. Second, integrin-mediated activation of Rac may lead to the activation of PAK, promoting the phosphorylation of either Raf at serine 338 (King et al., 1998) and/or MEK at serine 298 (Slack-Davis et al., 2003b). Either pathway would lead to the subsequent activation of the ERK pathway. This model also predicts that failure to either activate FAK or localize RACK 1 would 
result in the attenuation of integrin-dependent activation of Raf or MEK respectively and decrease adhesion disassembly rates (Webb et al., 2004). Therefore, localized events that negatively regulate FAK activity or prevent the localization of RACK 1 would fail to positively regulate ERK activation at newly formed adhesions and allow the adhesions to persist for longer times.

More persistent (mature) adhesions are predominantly found in the trailing edge of polarized fibroblasts (Rid et al., 2005). In contrast to de novo formed stationary front adhesions, these adhesions appear to be elongated and mobile (Smilenov et al., 1999). Interestingly, activated ERK staining in these adhesions seems to be less prominent than in leading edge adhesions (our unpublished observations). Thus we argue the possibility that signals controlling mobility/treadmilling of the adhesions found in the trailing edge differ from the signals controlling leading edge adhesion disassembly.

To test the model presented in this thesis, there is a need for further experiments addressing the issue of contribution of the Rho pathway to the regulation of disassembly rates of leading edge adhesions. We do not know if PDZ-RhoGEF and/or ROCK II contribute to leading edge adhesion disassembly and localization of active ERK to these adhesions. We speculate that the localization of active ERK and rates of leading edge adhesion disassembly should not depend on PDZ-RhoGEF or ROCK II. However, one possible outcome is that the absence of PDZ-RhoGEF or ROCK II may not alter integrinmediated ERK activation or active ERK localization to adhesion structures but still inhibits leading edge adhesion disassembly. If this result is observed, it would indicate that there is an ERK-independent but Rho-dependent mechanism to regulate adhesion disassembly at the front, or that both pathways are necessary.

Our preliminary experiments indicate that pharmacological inhibition of MEK did not prevent LPA from stimulating adhesion movement in serum starved cells. These 
preliminary observations argue that activation of ERK may not contribute significantly to controlling mature adhesion mobility. RhoA activation has been shown to occur at the leading edge of migrating fibroblasts (Pertz et al., 2006). In addition, RhoA effector mDia2 has been implicated in the regulation of adhesion dynamics in the lamellipodia of epithelial cells (Gupton et al., 2007). These data indicated that the activation of the RhoA/mDia2 pathway in the lamellipodia couples to leading edge adhesion turn-over. It is possible that PDZ-RhoGEF activates the RhoA/mDia2 pathway in the lamellipodia, thus participating in the regulation of leading edge adhesion turnover.

The work presented in this thesis places Focal Adhesion Kinase as a central initiator of different signaling pathways that spatially regulate adhesion dynamics in polarized fibroblasts. FAK has been shown to be involved in the regulation of cell migration (Parsons et al., 2008). Interestingly cancer cell types that exhibit fibroblastic migration show increased activation of FAK (McLean et al., 2005). Therefore it is reasonable to think that interference with FAK signaling to the ERK or the Rho pathway could modulate migration of selected cancer cells.

Migration of fibrosarcoma cells (HT1080) in vivo is characterized by formation of front protrusions and retraction of the trailing edge, a phenotype very similar to fibroblast migration in tissue culture conditions (Peter Friedl, Cell migration in Invasion and Inflamation, Keystone 2008). Interestingly, HT1080 cells show increased phosphorylation of FAK and FAK activation-dependent matrigel invasion (Park et al., 2005). Hyper-phosphorylation of FAK in HT1080 cells correlates with sustained Rho activation (Mukai et al., 2002). Furthermore, HT1080 fibrosarcoma cells possess oncogenic mutant alleles of N-Ras (Gupta et al., 2001) that constitutively activate the MAP kinase pathway. Inhibition of the MAP kinase pathway with a MEK inhibitor stops matrigel invasion of HT1080 cell, suggesting the involvement of the ERK in migration of 
these cells (our observations). Thus, it is attractive to hypothesize that changes in FAKdependent regulation of ERK and Rho pathways at adhesion structures regulate the metastatic potential of HT1080 cells. The use of advanced imaging techniques combined with genetic approaches will be necessary to address the issues of the in vivo relevance of FAK signaling to MAP kinase and Rho in the regulation of cell migration. 


\section{Figures}

Figure 33. Proposed model of how FAK could spatially regulate adhesion dynamics in the lamellipodia and trailing edge of migrationg cells.

In the lamellipodia, integrin engagement with substrate induces recruitment of FAK to cytoplasmic portion of $\beta 1$ integrin subunits. FAK phosphorylates itself at tyrosine 397 and recruits Src to form a bi-partite complex. In turn Src phosphorylates FAK at tyrosine 925 which serves as a docking site for the adaptor protein Grb2. Grb2, by recruitment of SOS initiates Ras signaling to activate the ERK pathway. Alternatively, Src can phosphorylate Raf at tyrosine 340/341 and initiate Raf dependent activation of the ERK pathway. In addition to Src, MP1/PAK complex is activated by integrindependent Rac activation. Activated PAK phosphorylates Raf at serine 338 and MEK and serine 298 and initiates the ERK pathway. Independently of FAK, the MAP kinase scaffold RACK1 is recruited to integrins and recruits the ERK (Raf,MEK, ERK) pathway to FAK for activation. In turn active ERK via downstream unknown molecules initiates adhesion disassembly.

In the trailing edge PDZ-RhoGEF that is present on actin filaments links LPA receptor activation with adhesion dynamics. PDZ-RhoGEF, via its RGS domain, binds to the GTP-loaded $\alpha$ subunit of heterotrimeric G proteins and subsequently Rho A. The FAK/Src complex phosphorylates PDZ-RhoGEF and leads to PDZ-RhoGEF-mediated activation of Rho. Rho binds to ROCK II and activates it. ROCK II, via unknown mechanisms controls adhesion movement and trailing edge retraction. 
Figure 33.
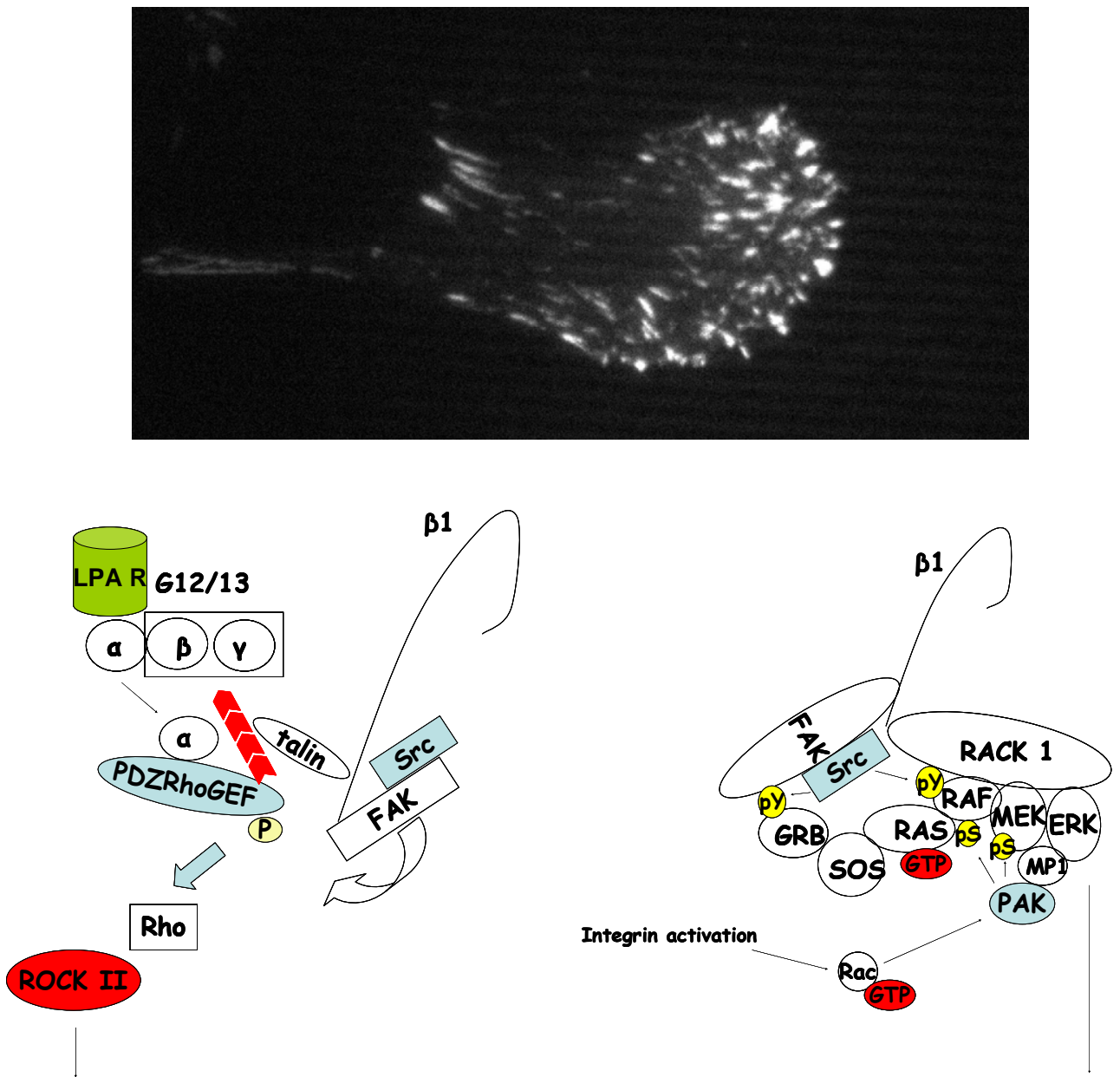

Adhesion movement

Adhesion disassembly 
References.

Abercrombie, M. and Dunn, G. A. (1975). Adhesions of fibroblasts to substratum during contact inhibition observed by interference reflection microscopy. Exp Cell Res 92, 57-62.

Adams, M. D. Celniker, S. E. Holt, R. A. Evans, C. A. Gocayne, J. D. Amanatides, P. G. Scherer, S. E. Li, P. W. Hoskins, R. A. Galle, R. F. et al. (2000). The Genome Sequence of Drosophila melanogaster 10.1126/science.287.5461.2185. Science, 2185-2195.

Agochiya, M., Brunton, V. G., Owens, D. W., Parkinson, E. K., Paraskeva, C., Keith, W. N. and Frame, M. C. (1999). Increased dosage and amplification of the focal adhesion kinase gene in human cancer cells. Oncogene 18, 5646-53.

Akagi, T., Murata, K., Shishido, T. and Hanafusa, H. (2002). v-Crk activates the phosphoinositide 3-kinase/AKT pathway by utilizing focal adhesion kinase and H-Ras. Mol Cell Biol 22, 7015-23.

Allen, C. D., Okada, T. and Cyster, J. G. (2007). Germinal-center organization and cellular dynamics. Immunity 27, 190-202.

Amano, M., Ito, M., Kimura, K., Fukata, Y., Chihara, K., Nakano, T., Matsuura, Y. and Kaibuchi, K. (1996a). Phosphorylation and activation of myosin by Rho-associated kinase (Rho-kinase). Journal of Biological Chemistry 271, 20246-9.

Amano, M., Ito, M., Kimura, K., Fukata, Y., Chihara, K., Nakano, T., Matsuura, Y. and Kaibuchi, K. (1996b). Phosphorylation and activation of myosin by Rho-associated kinase (Rho-kinase). J Biol Chem 271, 20246-9. 
Anderson, R., Copeland, T. K., Scholer, H., Heasman, J. and Wylie, C. (2000). The onset of germ cell migration in the mouse embryo. Mech Dev 91, 61-8. Andrianantoandro, E. and Pollard, T. D. (2006). Mechanism of actin filament turnover by severing and nucleation at different concentrations of ADF/cofilin. Mol Cell 24, 13-23.

Arias-Salgado, E. G., Lizano, S., Sarkar, S., Brugge, J. S., Ginsberg, M. H. and Shattil, S. J. (2003). Src kinase activation by direct interaction with the integrin beta cytoplasmic domain. Proc Natl Acad Sci U S A 100, 13298-302.

Astrof, S., Crowley, D. and Hynes, R. O. (2007). Multiple cardiovascular defects caused by the absence of alternatively spliced segments of fibronectin. Dev Biol 311, 11-24.

Aumailley, M., Bruckner-Tuderman, L., Carter, W. G., Deutzmann, R., Edgar, D., Ekblom, P., Engel, J., Engvall, E., Hohenester, E., Jones, J. C. et al. (2005). A simplified laminin nomenclature. Matrix Biol 24, 326-32.

Azuma, T., Witke, W., Stossel, T. P., Hartwig, J. H. and Kwiatkowski, D. J. (1998). Gelsolin is a downstream effector of rac for fibroblast motility. Embo $\mathrm{J} 17$, 1362-70.

Babcock, L. E. (1988). New Permian conulariids from Devon Island, Canada. Journal Of Paleontology 62, 615.

Balasubramanian, N., Scott, D. W., Castle, J. D., Casanova, J. E. and Schwartz, M. A. (2007). Arf6 and microtubules in adhesion-dependent trafficking of lipid rafts. Nat Cell Biol 9, 1381-91.

Banerjee, J. and Wedegaertner, P. B. (2004). Identification of a novel sequence in PDZ-RhoGEF that mediates interaction with the actin cytoskeleton. Mol Biol Cell 15, 1760-75. 
Barkalow, K., Witke, W., Kwiatkowski, D. J. and Hartwig, J. H. (1996). Coordinated regulation of platelet actin filament barbed ends by gelsolin and capping protein. J Cell Biol 134, 389-99.

Barr, A. J., Brass, L. F. and Manning, D. R. (1997). Reconstitution of receptors and GTP-binding regulatory proteins (G proteins) in Sf9 cells. A direct evaluation of selectivity in receptor.G protein coupling. J Biol Chem 272, 2223-9.

Bartolini, F., Moseley, J. B., Schmoranzer, J., Cassimeris, L., Goode, B. L. and Gundersen, G. G. (2008). The formin mDia2 stabilizes microtubules independently of its actin nucleation activity. J Cell Biol 181, 523-36.

Beggs, H. E., Schahin-Reed, D., Zang, K., Goebbels, S., Nave, K. A., Gorski, J., Jones, K. R., Sretavan, D. and Reichardt, L. F. (2003). FAK deficiency in cells contributing to the basal lamina results in cortical abnormalities resembling congenital muscular dystrophies. Neuron 40, 501-14.

Bian, D., Mahanivong, C., Yu, J., Frisch, S. M., Pan, Z. K., Ye, R. D. and Huang, S. (2006). The G12/13-RhoA signaling pathway contributes to efficient lysophosphatidic acid-stimulated cell migration. Oncogene 25, 2234-44.

Bishop, A. L. and Hall, A. (2000). Rho GTPases and their effector proteins. Biochem J 348 Pt 2, 241-55.

Boggon, T. J. and Eck, M. J. (2004). Structure and regulation of Src family kinases. Oncogene 23, 7918-27.

Borisy, G. G. and Svitkina, T. M. (2000). Actin machinery: pushing the envelope. Curr Opin Cell Biol 12, 104-12.

Bork, P., Downing, A. K., Kieffer, B. and Campbell, I. D. (1996). Structure and distribution of modules in extracellular proteins. $Q$ Rev Biophys 29, 119-67. 
Bourett, T. M., Czymmek, K. J. and Howard, R. J. (1998). An improved method for affinity probe localization in whole cells of filamentous fungi. Fungal Genetics \& Biology 24, 3-13.

Braren, R., Hu, H., Kim, Y. H., Beggs, H. E., Reichardt, L. F. and Wang, R. (2006). Endothelial FAK is essential for vascular network stability, cell survival, and lamellipodial formation. J Cell Biol 172, 151-62.

Bresnick, A. R. (1999). Molecular mechanisms of nonmuscle myosin-II regulation. Curr Opin Cell Biol 11, 26-33.

Brugge, J. S. and Erikson, R. L. (1977). Identification of a transformationspecific antigen induced by an avian sarcoma virus. Nature 269 , 346-8.

Brugge, J. S., Steinbaugh, P. J. and Erikson, R. L. (1978). Characterization of the avian sarcoma virus protein p60src. Virology 91, 130-40.

Brugnera, E., Haney, L., Grimsley, C., Lu, M., Walk, S. F., Tosello-Trampont, A. C., Macara, I. G., Madhani, H., Fink, G. R. and Ravichandran, K. S. (2002). Unconventional Rac-GEF activity is mediated through the Dock180-ELMO complex. Nat Cell Biol 4, 574-82.

Brunton, V. G., MacPherson, I. R. and Frame, M. C. (2004). Cell adhesion receptors, tyrosine kinases and actin modulators: a complex three-way circuitry. Biochim Biophys Acta 1692, 121-44.

Burke, R. D. (1999). Invertebrate integrins: structure, function, and evolution. Int Rev Cytol 191, 257-84.

Burnham, M. R., Harte, M. T., Richardson, A., Parsons, J. T. and Bouton, A. H. (1996). The identification of p130cas-binding proteins and their role in cellular transformation. Oncogene 12, 2467-72. 
Burridge, K., Fath, K., Kelly, T., Nuckolls, G. and Turner, C. (1988). Focal adhesions: transmembrane junctions between the extracellular matrix and the cytoskeleton. Annu Rev Cell Biol 4, 487-525.

Burridge, K., Molony, L. and Kelly, T. (1987). Adhesion plaques: sites of transmembrane interaction between the extracellular matrix and the actin cytoskeleton. J Cell Sci Suppl 8, 211-29.

Cai, X., Lietha, D., Ceccarelli, D. F., Karginov, A. V., Rajfur, Z., Jacobson, K., Hahn, K. M., Eck, M. J. and Schaller, M. D. (2007). Spatial and Temporal Regulation of FAK Activity in Living Cells

10.1128/MCB.01324-07. Mol. Cell. Biol., MCB.01324-07.

Cance, W. G., Harris, J. E., lacocca, M. V., Roche, E., Yang, X., Chang, J., Simkins, S. and Xu, L. (2000). Immunohistochemical analyses of focal adhesion kinase expression in benign and malignant human breast and colon tissues: correlation with preinvasive and invasive phenotypes. Clin Cancer Res 6, 2417-23.

Carragher, N. O., Westhoff, M. A., Fincham, V. J., Schaller, M. D. and Frame, M. C. (2003). A novel role for FAK as a protease-targeting adaptor protein: regulation by p42 ERK and Src. Curr Biol 13, 1442-50.

Carrell, N. A., Fitzgerald, L. A., Steiner, B., Erickson, H. P. and Phillips, D. R. (1985). Structure of human platelet membrane glycoproteins IIb and IIIa as determined by electron microscopy. J Biol Chem 260, 1743-9.

Cassimeris, L., Safer, D., Nachmias, V. T. and Zigmond, S. H. (1992). Thymosin beta 4 sequesters the majority of G-actin in resting human polymorphonuclear leukocytes. J Cell Biol 119, 1261-70.

Chang, B. Y., Conroy, K. B., Machleder, E. M. and Cartwright, C. A. (1998). RACK1, a receptor for activated C kinase and a homolog of the beta subunit of G 
proteins, inhibits activity of src tyrosine kinases and growth of NIH 3 T3 cells. Mol Cell Biol 18, 3245-56.

Chang, W., Gruber, D., Chari, S., Kitazawa, H., Hamazumi, Y., Hisanaga, S. and Bulinski, J. C. (2001). Phosphorylation of MAP4 affects microtubule properties and cell cycle progression. Journal of Cell Science 114, 2879-87.

Chen, H. C., Appeddu, P. A., Isoda, H. and Guan, J. L. (1996). Phosphorylation of tyrosine 397 in focal adhesion kinase is required for binding phosphatidylinositol 3-kinase. J Biol Chem 271, 26329-34.

Chen, H. C., Appeddu, P. A., Parsons, J. T., Hildebrand, J. D., Schaller, M. D. and Guan, J. L. (1995). Interaction of focal adhesion kinase with cytoskeletal protein talin. Journal of Biological Chemistry 270, 16995-9.

Chen, Q., Kinch, M. S., Lin, T. H., Burridge, K. and Juliano, R. L. (1994). Integrin-mediated cell adhesion activates mitogen-activated protein kinases. $J$ Biol Chem 269, 26602-5.

Chikumi, H., Barac, A., Behbahani, B., Gao, Y., Teramoto, H., Zheng, Y. and Gutkind, J. S. (2004). Homo- and hetero-oligomerization of PDZ-RhoGEF, LARG and p115RhoGEF by their C-terminal region regulates their in vivo Rho GEF activity and transforming potential. Oncogene 23, 233-40.

Chikumi, H., Fukuhara, S. and Gutkind, J. S. (2002). Regulation of G proteinlinked guanine nucleotide exchange factors for Rho, PDZ-RhoGEF, and LARG by tyrosine phosphorylation: evidence of a role for focal adhesion kinase. $J$ Biol Chem 277, 12463-73.

Chipperfield, M. P. and Pyle, J. A. (1988). 2-dimensional modelling of the antarctic lower stratosphere. Geophysical Research Letters 15, 875. 
Colognato, H. and Yurchenco, P. D. (2000). Form and function: the laminin family of heterotrimers. Dev Dyn 218, 213-34.

Conti, M. A. and Adelstein, R. S. (2008). Nonmuscle myosin II moves in new directions. J Cell Sci 121, 11-8.

Contos, J. J., Fukushima, N., Weiner, J. A., Kaushal, D. and Chun, J. (2000). Requirement for the IpA1 lysophosphatidic acid receptor gene in normal suckling behavior. Proc Natl Acad Sci U S A 97, 13384-9.

Cook, D. N. and Bottomly, K. (2007). Innate immune control of pulmonary dendritic cell trafficking. Proc Am Thorac Soc 4, 234-9.

Cordell, B., Weiss, S. R., Varmus, H. E. and Bishop, J. M. (1978). At least 104 nucleotides are transposed from the 5' terminus of the avian sarcoma virus genome to the $5^{\prime}$ termini of smaller viral mRNAs. Cell 15, 79-91.

Cukierman, E., Pankov, R., Stevens, D. R. and Yamada, K. M. (2001). Taking cell-matrix adhesions to the third dimension. Science 294, 1708-12.

Czuchra, A., Wu, X., Meyer, H., van Hengel, J., Schroeder, T., Geffers, R., Rottner, K. and Brakebusch, C. (2005). Cdc42 is not essential for filopodium formation, directed migration, cell polarization, and mitosis in fibroblastoid cells. Mol Biol Cell 16, 4473-84.

Davidson, L. A. and Keller, R. E. (1999). Neural tube closure in Xenopus laevis involves medial migration, directed protrusive activity, cell intercalation and convergent extension. Development 126, 4547-56.

del Pozo, M. A., Alderson, N. B., Kiosses, W. B., Chiang, H. H., Anderson, R. G. and Schwartz, M. A. (2004). Integrins regulate Rac targeting by internalization of membrane domains. Science 303, 839-42. 
del Pozo, M. A., Balasubramanian, N., Alderson, N. B., Kiosses, W. B., Grande-Garcia, A., Anderson, R. G. and Schwartz, M. A. (2005). Phospho-caveolin1 mediates integrin-regulated membrane domain internalization. Nat Cell Biol 7, 901-8.

Del Pozo, M. A., Kiosses, W. B., Alderson, N. B., Meller, N., Hahn, K. M. and Schwartz, M. A. (2002). Integrins regulate GTP-Rac localized effector interactions through dissociation of Rho-GDI. Nature Cell Biology 4, 232-9.

del Pozo, M. A., Schwartz, M. A., Hu, J., Kiosses, W. B., Altman, A. and Villalba, M. (2003). Guanine exchange-dependent and -independent effects of Vav1 on integrin-induced T cell spreading. J Immunol 170, 41-7.

Della Rocca, G. J., Maudsley, S., Daaka, Y., Lefkowitz, R. J. and Luttrell, L. M. (1999). Pleiotropic coupling of G protein-coupled receptors to the mitogenactivated protein kinase cascade. Role of focal adhesions and receptor tyrosine kinases. J Biol Chem 274, 13978-84.

Di Paolo, G. and De Camilli, P. (2006). Phosphoinositides in cell regulation and membrane dynamics. Nature 443, 651-7.

Dibb, N. J., Dilworth, S. M. and Mol, C. D. (2004). Switching on kinases: oncogenic activation of BRAF and the PDGFR family. Nat Rev Cancer 4, 718-27.

Digman, M. A., Brown, C. M., Horwitz, A. R., Mantulin, W. W. and Gratton, E. (2008). Paxillin dynamics measured during adhesion assembly and disassembly by correlation spectroscopy. Biophys $\mathrm{J} 94,2819-31$.

Drabek, K., van Ham, M., Stepanova, T., Draegestein, K., van Horssen, R., Sayas, C. L., Akhmanova, A., Ten Hagen, T., Smits, R., Fodde, R. et al. (2006). Role of CLASP2 in microtubule stabilization and the regulation of persistent motility. Curr Biol 16, 2259-64. 
Drenckhahn, D. and Pollard, T. D. (1986). Elongation of actin filaments is a diffusion-limited reaction at the barbed end and is accelerated by inert macromolecules. J Biol Chem 261, 12754-8.

Du, X., Gu, M., Weisel, J. W., Nagaswami, C., Bennett, J. S., Bowditch, R. and Ginsberg, M. H. (1993). Long range propagation of conformational changes in integrin alpha IIb beta 3. J Biol Chem 268, 23087-92.

El Fahime, E., Torrente, Y., Caron, N. J., Bresolin, M. D. and Tremblay, J. P. (2000). In vivo migration of transplanted myoblasts requires matrix metalloproteinase activity. Exp Cell Res 258, 279-87.

Essayem, S., Kovacic-Milivojevic, B., Baumbusch, C., McDonagh, S., Dolganov, G., Howerton, K., Larocque, N., Mauro, T., Ramirez, A., Ramos, D. M. et al. (2006). Hair cycle and wound healing in mice with a keratinocyte-restricted deletion of FAK. Oncogene 25, 1081-9.

Euteneuer, U. and Schliwa, M. (1984). Persistent, directional motility of cells and cytoplasmic fragments in the absence of microtubules. Nature 310, 58-61.

Ezratty, E. J., Partridge, M. A. and Gundersen, G. G. (2005). Microtubuleinduced focal adhesion disassembly is mediated by dynamin and focal adhesion kinase. Nat Cell Biol 7, 581-90.

Farina, K. L., Wyckoff, J. B., Rivera, J., Lee, H., Segall, J. E., Condeelis, J. S. and Jones, J. G. (1998). Cell motility of tumor cells visualized in living intact primary tumors using green fluorescent protein. Cancer Res 58, 2528-32.

Fassler, R., Pfaff, M., Murphy, J., Noegel, A. A., Johansson, S., Timpl, R. and Albrecht, R. (1995). Lack of beta 1 integrin gene in embryonic stem cells affects morphology, adhesion, and migration but not integration into the inner cell mass of blastocysts. J Cell Biol 128, 979-88. 
Faull, R. J. and Ginsberg, M. H. (1996). Inside-out signaling through integrins. J Am Soc Nephrol 7, 1091-7.

Fincham, V. J., James, M., Frame, M. C. and Winder, S. J. (2000). Active ERK/MAP kinase is targeted to newly forming cell-matrix adhesions by integrin engagement and v-Src. EMBO J 19, 2911-23.

Francis, S. A., Shen, X., Young, J. B., Kaul, P. and Lerner, D. J. (2006). Rho GEF Lsc is required for normal polarization, migration, and adhesion of formylpeptide-stimulated neutrophils. Blood 107, 1627-35.

Franco, S. J., Rodgers, M. A., Perrin, B. J., Han, J., Bennin, D. A., Critchley, D. R. and Huttenlocher, A. (2004). Calpain-mediated proteolysis of talin regulates adhesion dynamics. Nat Cell Biol 6, 977-83.

Friedl, P., Maaser, K., Klein, C. E., Niggemann, B., Krohne, G. and Zanker, K. S. (1997). Migration of highly aggressive MV3 melanoma cells in 3-dimensional collagen lattices results in local matrix reorganization and shedding of alpha2 and beta1 integrins and CD44. Cancer Res 57, 2061-70.

Friedl, P., Noble, P. B., Walton, P. A., Laird, D. W., Chauvin, P. J., Tabah, R. J., Black, M. and Zanker, K. S. (1995). Migration of coordinated cell clusters in mesenchymal and epithelial cancer explants in vitro. Cancer Res 55, 4557-60.

Friedl, P. and Wolf, K. (2003). Tumour-cell invasion and migration: diversity and escape mechanisms. Nat Rev Cancer 3, 362-74.

Fuchs, E. (2007). Scratching the surface of skin development. Nature 445, 834-42.

Fukuhara, S., Murga, C., Zohar, M., Igishi, T. and Gutkind, J. S. (1999). A novel PDZ domain containing guanine nucleotide exchange factor links heterotrimeric G proteins to Rho. J Biol Chem 274, 5868-79. 
Gabarra-Niecko, V., Schaller, M. D. and Dunty, J. M. (2003). FAK regulates biological processes important for the pathogenesis of cancer. Cancer Metastasis Rev 22, 359-74.

Gailit, J. and Ruoslahti, E. (1988). Regulation of the fibronectin receptor affinity by divalent cations. J Biol Chem 263, 12927-32.

Garcia-Alvarez, B., de Pereda, J. M., Calderwood, D. A., Ulmer, T. S., Critchley, D., Campbell, I. D., Ginsberg, M. H. and Liddington, R. C. (2003). Structural determinants of integrin recognition by talin. Mol Cell 11, 49-58.

Gardner, H., Kreidberg, J., Koteliansky, V. and Jaenisch, R. (1996). Deletion of integrin alpha 1 by homologous recombination permits normal murine development but gives rise to a specific deficit in cell adhesion. Dev Biol 175, 30113.

George, E., Georges-Labouesse, E., Patel-King, R., Rayburn, H. and Hynes, R. (1993). Defects in mesoderm, neural tube and vascular development in mouse embryos lacking fibronectin. Development 119, 1079-1091.

Georges-Labouesse, E., Messaddeq, N., Yehia, G., Cadalbert, L., Dierich, A. and Le Meur, M. (1996). Absence of integrin alpha 6 leads to epidermolysis bullosa and neonatal death in mice. Nat Genet 13, 370-3.

Ghosh, M., Song, X., Mouneimne, G., Sidani, M., Lawrence, D. S. and Condeelis, J. S. (2004). Cofilin promotes actin polymerization and defines the direction of cell motility. Science 304, 743-6.

Girault, J. A., Labesse, G., Mornon, J. P. and Callebaut, I. (1999). The Ntermini of FAK and JAKs contain divergent band 4.1 domains. Trends Biochem Sci 24, 54-7. 
Glading, A., Bodnar, R. J., Reynolds, I. J., Shiraha, H., Satish, L., Potter, D. A., Blair, H. C. and Wells, A. (2004). Epidermal growth factor activates m-calpain (calpain II), at least in part, by extracellular signal-regulated kinase-mediated phosphorylation. Mol Cell Biol 24, 2499-512.

Gohla, A., Harhammer, R. and Schultz, G. (1998). The G-protein G13 but not G12 mediates signaling from lysophosphatidic acid receptor via epidermal growth factor receptor to Rho. J Biol Chem 273, 4653-9.

Goldschmidt-Clermont, P. J., Furman, M. I., Wachsstock, D., Safer, D., Nachmias, V. T. and Pollard, T. D. (1992). The control of actin nucleotide exchange by thymosin beta 4 and profilin. A potential regulatory mechanism for actin polymerization in cells. Mol Biol Cell 3, 1015-24.

Goldschmidt-Clermont, P. J., Machesky, L. M., Doberstein, S. K. and Pollard, T. D. (1991). Mechanism of the interaction of human platelet profilin with actin. J Cell Biol 113, 1081-9.

Golubovskaya, V. M., Finch, R., Kweh, F., Massoll, N. A., CampbellThompson, M., Wallace, M. R. and Cance, W. G. (2007). p53 regulates FAK expression in human tumor cells. Mol Carcinog.

Gotwals, P., Fessler, L., Wehrli, M. and Hynes, R. (1994). Drosophila PS1 Integrin is a Laminin Receptor and Differs in Ligand Specificity from PS2 10.1073/pnas.91.24.11447. PNAS 91, 11447-11451.

Grande-Garcia, A., Echarri, A., de Rooij, J., Alderson, N. B., WatermanStorer, C. M., Valdivielso, J. M. and del Pozo, M. A. (2007). Caveolin-1 regulates cell polarization and directional migration through Src kinase and Rho GTPases. J Cell Biol 177, 683-94. 
Guan, J. L., Trevithick, J. E. and Hynes, R. O. (1991). Fibronectin/integrin interaction induces tyrosine phosphorylation of a 120-kDa protein. Cell Regul 2, 951-64.

Gundersen, G. G., Kreitzer, G., Cook, T. and Liao, G. (1998). Microtubules as determinants of cellular polarity. Biological Bulletin 194, 358-60.

Gupta, S., Stuffrein, S., Plattner, R., Tencati, M., Gray, C., Whang, Y. E. and Stanbridge, E. J. (2001). Role of phosphoinositide 3-kinase in the aggressive tumor growth of HT1080 human fibrosarcoma cells. Mol Cell Biol 21, 5846-56.

Gupton, S. L., Eisenmann, K., Alberts, A. S. and Waterman-Storer, C. M. (2007). mDia2 regulates actin and focal adhesion dynamics and organization in the lamella for efficient epithelial cell migration. J Cell Sci 120, 3475-87.

Hakim, Z. S., DiMichele, L. A., Doherty, J. T., Homeister, J. W., Beggs, H. E., Reichardt, L. F., Schwartz, R. J., Brackhan, J., Smithies, O., Mack, C. P. et al. (2007). Conditional deletion of focal adhesion kinase leads to defects in ventricular septation and outflow tract alignment. Mol Cell Biol 27, 5352-64.

Han, D. C. and Guan, J. L. (1999). Association of focal adhesion kinase with Grb7 and its role in cell migration. J Biol Chem 274, 24425-30.

Hanks, S. K., Calalb, M. B., Harper, M. C. and Patel, S. K. (1992). Focal adhesion protein-tyrosine kinase phosphorylated in response to cell attachment to fibronectin. Proc Natl Acad Sci U S A 89, 8487-91.

Hannigan, G. E., Leung-Hagesteijn, C., Fitz-Gibbon, L., Coppolino, M. G., Radeva, G., Filmus, J., Bell, J. C. and Dedhar, S. (1996). Regulation of cell adhesion and anchorage-dependent growth by a new beta 1-integrin-linked protein kinase. Nature 379, 91-6. 
Harte, M. T., Hildebrand, J. D., Burnham, M. R., Bouton, A. H. and Parsons, J. T. (1996). p130Cas, a substrate associated with v-Src and v-Crk, localizes to focal adhesions and binds to focal adhesion kinase. J Biol Chem 271, 13649-55.

Heath, J. P. and Dunn, G. A. (1978). Cell to substratum contacts of chick fibroblasts and their relation to the microfilament system. A correlated interference-reflexion and high-voltage electron-microscope study. J Cell Sci 29, 197-212.

Hegerfeldt, Y., Tusch, M., Brocker, E. B. and FriedI, P. (2002). Collective cell movement in primary melanoma explants: plasticity of cell-cell interaction, beta1integrin function, and migration strategies. Cancer Res 62, 2125-30.

Hildebrand, J. D., Schaller, M. D. and Parsons, J. T. (1993). Identification of sequences required for the efficient localization of the focal adhesion kinase, pp125FAK, to cellular focal adhesions. Journal of Cell Biology 123, 993-1005.

Hildebrand, J. D., Taylor, J. M. and Parsons, J. T. (1996). An SH3 domaincontaining GTPase-activating protein for Rho and Cdc42 associates with focal adhesion kinase. Molecular \& Cellular Biology 16, 3169-78.

Hogg, N., Clive Landis, R., Bates, P. A., Stanley, P. and Randi, A. M. (1994). The sticking point: How integrins bind to their ligands. Trends Cell Biol 4, 379-82.

Hohenester, E. and Engel, J. (2002). Domain structure and organisation in extracellular matrix proteins. Matrix Biol 21, 115-28.

Hood, J. D., Frausto, R., Kiosses, W. B., Schwartz, M. A. and Cheresh, D. A. (2003). Differential alphav integrin-mediated Ras-ERK signaling during two pathways of angiogenesis. J Cell Biol 162, 933-43.

Horne, W. A., Abdel-Ghany, M., Racker, E., Weiland, G. A., Oswald, R. E. and Cerione, R. A. (1988). Functional reconstitution of skeletal muscle Ca2+ 
channels: separation of regulatory and channel components. Proc Natl Acad Sci U $S$ A 85, 3718-22.

Horwitz, A., Duggan, K., Buck, C., Beckerle, M. C. and Burridge, K. (1986). Interaction of plasma membrane fibronectin receptor with talin--a transmembrane linkage. Nature 320, 531-3.

Horwitz, R. and Webb, D. (2003). Cell migration. Curr Biol 13, R756-9.

Hotulainen, P. and Lappalainen, P. (2006). Stress fibers are generated by two distinct actin assembly mechanisms in motile cells. J Cell Biol 173, 383-94.

Howard, J. and Hyman, A. A. (2003). Dynamics and mechanics of the microtubule plus end. Nature 422, 753-8.

Hsia, D. A., Mitra, S. K., Hauck, C. R., Streblow, D. N., Nelson, J. A., Ilic, D., Huang, S., Li, E., Nemerow, G. R., Leng, J. et al. (2003). Differential regulation of cell motility and invasion by FAK. Journal of Cell Biology 160, 753-67.

Hughes, P. E., Diaz-Gonzalez, F., Leong, L., Wu, C., McDonald, J. A., Shattil, S. J. and Ginsberg, M. H. (1996). Breaking the integrin hinge. A defined structural constraint regulates integrin signaling. J Biol Chem 271, 6571-4.

Hughes, P. E., Renshaw, M. W., Pfaff, M., Forsyth, J., Keivens, V. M., Schwartz, M. A. and Ginsberg, M. H. (1997). Suppression of integrin activation: a novel function of a Ras/Raf-initiated MAP kinase pathway. Cell 88, 521-30.

Huttenlocher, A., Palecek, S. P., Lu, Q., Zhang, W., Mellgren, R. L., Lauffenburger, D. A., Ginsberg, M. H. and Horwitz, A. F. (1997). Regulation of cell migration by the calcium-dependent protease calpain. J Biol Chem 272, 32719-22.

Hynes, R. O. (1992). Integrins: versatility, modulation, and signaling in cell adhesion. Cell 69, 11-25. 
Hynes, R. O. (1996). Targeted mutations in cell adhesion genes: what have we learned from them? Dev Biol 180, 402-12.

Hynes, R. O. and Yamada, K. M. (1982). Fibronectins: multifunctional modular glycoproteins. J Cell Biol 95, 369-77.

Hynes, R. O. and Zhao, Q. (2000). The Evolution of Cell Adhesion 10.1083/jcb.150.2.F89. J. Cell Biol. 150, 89F-96.

lijima, M., Huang, Y. E. and Devreotes, P. (2002). Temporal and spatial regulation of chemotaxis. Dev Cell 3, 469-78.

Ilic, D., Furuta, Y., Kanazawa, S., Takeda, N., Sobue, K., Nakatsuji, N., Nomura, S., Fujimoto, J., Okada, M. and Yamamoto, T. (1995). Reduced cell motility and enhanced focal adhesion contact formation in cells from FAKdeficient mice. Nature 377, 539-44.

Ilic, D., Kovacic, B., Johkura, K., Schlaepfer, D. D., Tomasevic, N., Han, Q., Kim, J. B., Howerton, K., Baumbusch, C., Ogiwara, N. et al. (2004). FAK promotes organization of fibronectin matrix and fibrillar adhesions. J Cell Sci 117, 177-87.

Ingber, D. E., Prusty, D., Frangioni, J. V., Cragoe, E. J., Jr., Lechene, C. and Schwartz, M. A. (1990). Control of intracellular pH and growth by fibronectin in capillary endothelial cells. J Cell Biol 110, 1803-11.

Ishibe, S., Joly, D., Liu, Z. X. and Cantley, L. G. (2004). Paxillin serves as an ERK-regulated scaffold for coordinating FAK and Rac activation in epithelial morphogenesis. Mol Cell 16, 257-67.

Ito, M., Nakano, T., Erdodi, F. and Hartshorne, D. J. (2004). Myosin phosphatase: structure, regulation and function. Mol Cell Biochem 259, 197-209. 
Itoh, K., Yoshioka, K., Akedo, H., Uehata, M., Ishizaki, T. and Narumiya, S. (1999). An essential part for Rho-associated kinase in the transcellular invasion of tumor cells. Nat Med 5, 221-5.

Jacques, T. S., Relvas, J. B., Nishimura, S., Pytela, R., Edwards, G. M., Streuli, C. H. and ffrench-Constant, C. (1998). Neural precursor cell chain migration and division are regulated through different beta1 integrins. Development 125, 3167-77.

Jalink, K., Eichholtz, T., Postma, F. R., van Corven, E. J. and Moolenaar, W. H. (1993). Lysophosphatidic acid induces neuronal shape changes via a novel, receptor-mediated signaling pathway: similarity to thrombin action. Cell Growth Differ 4, 247-55.

Jenkins, A. L., Nannizzi-Alaimo, L., Silver, D., Sellers, J. R., Ginsberg, M. H., Law, D. A. and Phillips, D. R. (1998). Tyrosine phosphorylation of the beta3 cytoplasmic domain mediates integrin-cytoskeletal interactions. J Biol Chem 273, 13878-85.

Johnson, L. N., Noble, M. E. and Owen, D. J. (1996). Active and inactive protein kinases: structural basis for regulation. Cell 85, 149-58.

Johnston, C. A. and Siderovski, D. P. (2007). Receptor-mediated activation of heterotrimeric G-proteins: current structural insights. Mol Pharmacol 72, 21930.

Kaiser, D. A., Vinson, V. K., Murphy, D. B. and Pollard, T. D. (1999). Profilin is predominantly associated with monomeric actin in Acanthamoeba. J Cell Sci 112 ( Pt 21), 3779-90. 
Kaverina, I., Krylyshkina, O. and Small, J. V. (1999). Microtubule targeting of substrate contacts promotes their relaxation and dissociation. Journal of Cell Biology 146, 1033-44.

Keller, R. (2002). Shaping the Vertebrate Body Plan by Polarized Embryonic Cell Movements

10.1126/science.1079478. Science 298, 1950-1954.

Keller, R. (2005). Cell migration during gastrulation. Curr Opin Cell Biol 17, 533-41.

Keller, R. (2006). Mechanisms of elongation in embryogenesis. Development 133, 2291-302.

Keller, R., Davidson, L., Edlund, A., Elul, T., Ezin, M., Shook, D. and Skoglund, P. (2000). Mechanisms of convergence and extension by cell intercalation. Philos Trans R Soc Lond B Biol Sci 355, 897-922.

Kim, J. B., Leucht, P., Luppen, C. A., Park, Y. J., Beggs, H. E., Damsky, C. H. and Helms, J. A. (2007). Reconciling the roles of FAK in osteoblast differentiation, osteoclast remodeling, and bone regeneration. Bone 41, 39-51.

Kimura, K., Ito, M., Amano, M., Chihara, K., Fukata, Y., Nakafuku, M., Yamamori, B., Feng, J., Nakano, T., Okawa, K. et al. (1996). Regulation of myosin phosphatase by Rho and Rho-associated kinase (Rho-kinase). Science 273, 245-8.

King, A. J., Sun, H., Diaz, B., Barnard, D., Miao, W., Bagrodia, S. and Marshall, M. S. (1998). The protein kinase Pak3 positively regulates Raf-1 activity through phosphorylation of serine 338. Nature 396, 180-3.

Kiosses, W. B., Shattil, S. J., Pampori, N. and Schwartz, M. A. (2001). Rac recruits high-affinity integrin alphavbeta3 to lamellipodia in endothelial cell migration. Nature Cell Biology 3, 316-20. 
Kirchhofer, D., Gailit, J., Ruoslahti, E., Grzesiak, J. and Pierschbacher, M. D. (1990). Cation-dependent changes in the binding specificity of the platelet receptor GPIIb/IIla. J Biol Chem 265, 18525-30.

Klemke, R. L., Cai, S., Giannini, A. L., Gallagher, P. J., de Lanerolle, P. and Cheresh, D. A. (1997). Regulation of cell motility by mitogen-activated protein kinase. J Cell Biol 137, 481-92.

Klinghoffer, R. A., Sachsenmaier, C., Cooper, J. A. and Soriano, P. (1999). Src family kinases are required for integrin but not PDGFR signal transduction. Embo J 18, 2459-71.

Kodama, A., Karakesisoglou, I., Wong, E., Vaezi, A. and Fuchs, E. (2003). ACF7: an essential integrator of microtubule dynamics. Cell 115, 343-54.

Kolch, W. (2005). Coordinating ERK/MAPK signalling through scaffolds and inhibitors. Nat Rev Mol Cell Biol 6, 827-37.

Kolch, W., Heidecker, G., Kochs, G., Hummel, R., Vahidi, H., Mischak, H., Finkenzeller, G., Marme, D. and Rapp, U. R. (1993). Protein kinase C alpha activates RAF-1 by direct phosphorylation. Nature 364, 249-52.

Kranenburg, O., Poland, M., van Horck, F. P., Drechsel, D., Hall, A. and Moolenaar, W. H. (1999). Activation of RhoA by lysophosphatidic acid and Galpha12/13 subunits in neuronal cells: induction of neurite retraction. Mol Biol Cell 10, 1851-7.

Kraus, A. C., Ferber, I., Bachmann, S. O., Specht, H., Wimmel, A., Gross, M. W., Schlegel, J., Suske, G. and Schuermann, M. (2002). In vitro chemo- and radioresistance in small cell lung cancer correlates with cell adhesion and constitutive activation of AKT and MAP kinase pathways. Oncogene 21, 8683-95. 
Krause, M., Dent, E. W., Bear, J. E., Loureiro, J. J. and Gertler, F. B. (2003). Ena/VASP proteins: regulators of the actin cytoskeleton and cell migration. Annu Rev Cell Dev Biol 19, 541-64.

Kraynov, V. S., Chamberlain, C., Bokoch, G. M., Schwartz, M. A., Slabaugh, S. and Hahn, K. M. (2000). Localized Rac activation dynamics visualized in living cells. Science $290,333-7$.

Kreidberg, J. A., Donovan, M. J., Goldstein, S. L., Rennke, H., Shepherd, K., Jones, R. C. and Jaenisch, R. (1996). Alpha 3 beta 1 integrin has a crucial role in kidney and lung organogenesis. Development 122, 3537-47.

Kwiatkowski, A. V., Rubinson, D. A., Dent, E. W., Edward van Veen, J., Leslie, J. D., Zhang, J., Mebane, L. M., Philippar, U., Pinheiro, E. M., Burds, A. A. et al. (2007). Ena/VASP Is Required for neuritogenesis in the developing cortex. Neuron 56, 441-55.

Kwiatkowski, D. J. (1999). Functions of gelsolin: motility, signaling, apoptosis, cancer. Curr Opin Cell Biol 11, 103-8.

Laird, D. J., von Andrian, U. H. and Wagers, A. J. (2008). Stem cell trafficking in tissue development, growth, and disease. Cell 132, 612-30.

Law, D. A., Nannizzi-Alaimo, L. and Phillips, D. R. (1996). Outside-in integrin signal transduction. Alpha IIb beta 3-(GP IIb IIIa) tyrosine phosphorylation induced by platelet aggregation. J Biol Chem 271, 10811-5.

Lebrand, C., Dent, E. W., Strasser, G. A., Lanier, L. M., Krause, M., Svitkina, T. M., Borisy, G. G. and Gertler, F. B. (2004). Critical role of Ena/VASP proteins for filopodia formation in neurons and in function downstream of netrin-1. Neuron 42, 37-49. 
Leung, T., Manser, E., Tan, L. and Lim, L. (1995). A novel serine/threonine kinase binding the Ras-related RhoA GTPase which translocates the kinase to peripheral membranes. J Biol Chem 270, 29051-4.

Liddington, R. C. and Ginsberg, M. H. (2002). Integrin activation takes shape. J Cell Biol 158, 833-9.

Lietha, D., Cai, X., Ceccarelli, D. F., Li, Y., Schaller, M. D. and Eck, M. J. (2007). Structural basis for the autoinhibition of focal adhesion kinase. Cell 129, 1177-87.

Liliental, J. and Chang, D. D. (1998). Rack1, a receptor for activated protein kinase C, interacts with integrin beta subunit. J Biol Chem 273, 2379-83.

Lim, Y., Lim, S. T., Tomar, A., Gardel, M., Bernard-Trifilo, J. A., Chen, X. L., Uryu, S. A., Canete-Soler, R., Zhai, J., Lin, H. et al. (2008). PyK2 and FAK connections to p190Rho guanine nucleotide exchange factor regulate RhoA activity, focal adhesion formation, and cell motility. J Cell Biol 180, 187-203.

Lin, S. X., Gundersen, G. G. and Maxfield, F. R. (2002). Export from pericentriolar endocytic recycling compartment to cell surface depends on stable, detyrosinated (glu) microtubules and kinesin. Molecular Biology of the Cell 13, 96109.

Liu, S., Calderwood, D. and Ginsberg, M. (2000a). Integrin cytoplasmic domain-binding proteins. J. Cell Sci. 113, 3563-3571.

Liu, S., Calderwood, D. A. and Ginsberg, M. H. (2000b). Integrin cytoplasmic domain-binding proteins. J Cell Sci 113 ( Pt 20), 3563-71.

Liu, Y., Loijens, J. C., Martin, K. H., Karginov, A. V. and Parsons, J. T. (2002). The association of ASAP1, an ADP ribosylation factor-GTPase activating 
protein, with focal adhesion kinase contributes to the process of focal adhesion assembly. Mol Biol Cell 13, 2147-56.

Luo, B. H., Carman, C. V. and Springer, T. A. (2007). Structural basis of integrin regulation and signaling. Annu Rev Immunol 25, 619-47.

Luttrell, L. M., Daaka, Y., Della Rocca, G. J. and Lefkowitz, R. J. (1997). G protein-coupled receptors mediate two functionally distinct pathways of tyrosine phosphorylation in rat $1 \mathrm{a}$ fibroblasts. Shc phosphorylation and receptor endocytosis correlate with activation of Erk kinases. J Biol Chem 272, 31648-56.

Luttrell, L. M., Daaka, Y. and Lefkowitz, R. J. (1999). Regulation of tyrosine kinase cascades by G-protein-coupled receptors. Curr Opin Cell Biol 11, 177-83.

Machesky, L. M., Atkinson, S. J., Ampe, C., Vandekerckhove, J. and Pollard, T. D. (1994). Purification of a cortical complex containing two unconventional actins from Acanthamoeba by affinity chromatography on profilin-agarose. $J$ Cell Biol 127, 107-15.

Majumdar, M., Seasholtz, T. M., Buckmaster, C., Toksoz, D. and Brown, J. H. (1999). A rho exchange factor mediates thrombin and Galpha(12)-induced cytoskeletal responses. J Biol Chem 274, 26815-21.

Martin, G. S. (2004). The road to Src. Oncogene 23, 7910-7.

Mayer, U., Saher, G., Fassler, R., Bornemann, A., Echtermeyer, F., von der Mark, H., Miosge, N., Poschl, E. and von der Mark, K. (1997). Absence of integrin alpha 7 causes a novel form of muscular dystrophy. Nat Genet 17, 318-23.

McLean, G. W., Carragher, N. O., Avizienyte, E., Evans, J., Brunton, V. G. and Frame, M. C. (2005). The role of focal-adhesion kinase in cancer - a new therapeutic opportunity. Nat Rev Cancer 5, 505-15. 
Meller, N., Irani-Tehrani, M., Kiosses, W. B., Del Pozo, M. A. and Schwartz, M. A. (2002). Zizimin1, a novel Cdc42 activator, reveals a new GEF domain for Rho proteins. Nature Cell Biology 4, 639-47.

Miki, H., Sasaki, T., Takai, Y. and Takenawa, T. (1998). Induction of filopodium formation by a WASP-related actin-depolymerizing protein N-WASP. Nature 391, 93-6.

Mills, G. B. and Moolenaar, W. H. (2003). The emerging role of lysophosphatidic acid in cancer. Nat Rev Cancer 3, 582-91.

Mitchison, T. and Kirschner, M. (1984). Dynamic instability of microtubule growth. Nature 312, 237-42.

Moll, R., Mitze, M., Frixen, U. H. and Birchmeier, W. (1993). Differential loss of E-cadherin expression in infiltrating ductal and lobular breast carcinomas. $\mathbf{A m}$ J Pathol 143, 1731-42.

Moolenaar, W. H., van Meeteren, L. A. and Giepmans, B. N. (2004). The ins and outs of lysophosphatidic acid signaling. Bioessays 26, 870-81.

Mor, A. and Philips, M. R. (2006). Compartmentalized Ras/MAPK signaling. Annu Rev Immunol 24, 771-800.

Morla, A. and Ruoslahti, E. (1992). A fibronectin self-assembly site involved in fibronectin matrix assembly: reconstruction in a synthetic peptide. $J$ Cell Biol 118, 421-9.

Morrison, D. K. and Davis, R. J. (2003). Regulation of MAP kinase signaling modules by scaffold proteins in mammals. Annu Rev Cell Dev Biol 19, 91-118.

Mukai, M., Togawa, A., Imamura, F., Iwasaki, T., Ayaki, M., Mammoto, T., Nakamura, H., Tatsuta, M. and Inoue, M. (2002). Sustained tyrosine- 
phosphorylation of FAK through Rho-dependent adhesion to fibronectin is essential for cancer cell migration. Anticancer Res 22, 3175-84.

Mullins, R. D., Heuser, J. A. and Pollard, T. D. (1998). The interaction of Arp2/3 complex with actin: nucleation, high affinity pointed end capping, and formation of branching networks of filaments. Proc Natl Acad Sci U S A 95, 6181-6.

Mundy, D. I., Machleidt, T., Ying, Y. S., Anderson, R. G. and Bloom, G. S. (2002). Dual control of caveolar membrane traffic by microtubules and the actin cytoskeleton. J Cell Sci 115, 4327-39.

Nabeshima, K., Inoue, T., Shimao, Y., Okada, Y., Itoh, Y., Seiki, M. and Koono, M. (2000). Front-cell-specific expression of membrane-type 1 matrix metalloproteinase and gelatinase A during cohort migration of colon carcinoma cells induced by hepatocyte growth factor/scatter factor. Cancer Res 60, 3364-9.

Nakagawa, O., Fujisawa, K., Ishizaki, T., Saito, Y., Nakao, K. and Narumiya, S. (1996). ROCK-I and ROCK-II, two isoforms of Rho-associated coiled-coil forming protein serine/threonine kinase in mice. FEBS Lett 392, 189-93.

Neptune, E. R. and Bourne, H. R. (1997). Receptors induce chemotaxis by releasing the betagamma subunit of Gi, not by activating Gq or Gs. Proc Natl Acad Sci U S A 94, 14489-94.

Nolte, M., Pepinsky, R. B., Venyaminov, S., Koteliansky, V., Gotwals, P. J. and Karpusas, M. (1999). Crystal structure of the alpha1beta1 integrin I-domain: insights into integrin I-domain function. FEBS Lett 452, 379-85.

Offermanns, S., Laugwitz, K. L., Spicher, K. and Schultz, G. (1994). G proteins of the G12 family are activated via thromboxane A2 and thrombin receptors in human platelets. Proc Natl Acad Sci U S A 91, 504-8. 
Otey, C. A., Pavalko, F. M. and Burridge, K. (1990). An interaction between alpha-actinin and the beta 1 integrin subunit in vitro. J Cell Biol 111, 721-9.

Owen, K. A., Pixley, F. J., Thomas, K. S., Vicente-Manzanares, M., Ray, B. J., Horwitz, A. F., Parsons, J. T., Beggs, H. E., Stanley, E. R. and Bouton, A. H. (2007). Regulation of lamellipodial persistence, adhesion turnover, and motility in macrophages by focal adhesion kinase. J Cell Biol 179, 1275-87.

Owens, L. V., Xu, L., Craven, R. J., Dent, G. A., Weiner, T. M., Kornberg, L., Liu, E. T. and Cance, W. G. (1995). Overexpression of the focal adhesion kinase (p125FAK) in invasive human tumors. Cancer Res 55, 2752-5.

Palazzo, A. F., Cook, T. A., Alberts, A. S. and Gundersen, G. G. (2001). mDia mediates Rho-regulated formation and orientation of stable microtubules. Nature Cell Biology 3, 723-9.

Palazzo, A. F., Eng, C. H., Schlaepfer, D. D., Marcantonio, E. E. and Gundersen, G. G. (2004). Localized stabilization of microtubules by integrin- and FAK-facilitated Rho signaling. Science 303, 836-9.

Palecek, S. P., Huttenlocher, A., Horwitz, A. F. and Lauffenburger, D. A. (1998). Physical and biochemical regulation of integrin release during rear detachment of migrating cells. J Cell Sci 111 ( Pt 7), 929-40.

Palecek, S. P., Schmidt, C. E., Lauffenburger, D. A. and Horwitz, A. F. (1996). Integrin dynamics on the tail region of migrating fibroblasts. J Cell Sci 109 ( Pt 5), 941-52.

Pankov, R. and Yamada, K. M. (2002). Fibronectin at a glance. J Cell Sci 115, 3861-3. 
Park, H., Han, I., Kwon, H. J. and Oh, E. S. (2005). Focal adhesion kinase regulates syndecan-2-mediated tumorigenic activity of HT1080 fibrosarcoma cells. Cancer Res 65, 9899-905.

Parsons, J. T. (2003). Focal adhesion kinase: the first ten years. J Cell Sci $116,1409-1416$.

Parsons, J. T., Schaller, M. D., Hildebrand, J., Leu, T. H., Richardson, A. and Otey, C. (1994). Focal adhesion kinase: structure and signalling. Journal of Cell Science - Supplement 18, 109-13.

Parsons, J. T., Slack-Davis, J., Tilghman, R. and Roberts, W. G. (2008). Focal adhesion kinase: targeting adhesion signaling pathways for therapeutic intervention. Clin Cancer Res 14, 627-32.

Parsons, S. J. and Parsons, J. T. (2004). Src family kinases, key regulators of signal transduction. Oncogene 23, 7906-9.

Pavalko, F. M., Otey, C. A. and Burridge, K. (1989). Identification of a filamin isoform enriched at the ends of stress fibers in chicken embryo fibroblasts. $J$ Cell Sci 94 ( Pt 1), 109-18.

Pearson, G., Robinson, F., Beers Gibson, T., Xu, B. E., Karandikar, M., Berman, K. and Cobb, M. H. (2001). Mitogen-activated protein (MAP) kinase pathways: regulation and physiological functions. Endocr Rev 22, 153-83.

Pellegrin, S. and Mellor, H. (2005). The Rho family GTPase Rif induces filopodia through mDia2. Curr Biol 15, 129-33.

Peng, X., Kraus, M. S., Wei, H., Shen, T. L., Pariaut, R., Alcaraz, A., Ji, G., Cheng, L., Yang, Q., Kotlikoff, M. I. et al. (2006). Inactivation of focal adhesion kinase in cardiomyocytes promotes eccentric cardiac hypertrophy and fibrosis in mice. J Clin Invest 116, 217-27. 
Pertz, O., Hodgson, L., Klemke, R. L. and Hahn, K. M. (2006). Spatiotemporal dynamics of RhoA activity in migrating cells. Nature 440, 1069-72.

Pitts, W. C., Rojas, V. A., Gaffey, M. J., Rouse, R. V., Esteban, J., Frierson, H. F., Kempson, R. L. and Weiss, L. M. (1991). Carcinomas with metaplasia and sarcomas of the breast. Am J Clin Pathol 95, 623-32.

Playford, M. P. and Schaller, M. D. (2004). The interplay between Src and integrins in normal and tumor biology. Oncogene 23, 7928-46.

Pollard, T. D. and Beltzner, C. C. (2002). Structure and function of the Arp2/3 complex. Curr Opin Struct Biol 12, 768-74.

Pollard, T. D. and Borisy, G. G. (2003). Cellular motility driven by assembly and disassembly of actin filaments. Cell 112, 453-65.

Potts, J. R. and Campbell, I. D. (1996). Structure and function of fibronectin modules. Matrix Biol 15, 313-20; discussion 321.

Pullikuth, A., McKinnon, E., Schaeffer, H. J. and Catling, A. D. (2005). The MEK1 scaffolding protein MP1 regulates cell spreading by integrating PAK1 and Rho signals. Mol Cell Biol 25, 5119-33.

Raftopoulou, M. and Hall, A. (2004). Cell migration: Rho GTPases lead the way. Dev Biol 265, 23-32.

Regen, C. M. and Horwitz, A. F. (1992). Dynamics of beta 1 integrinmediated adhesive contacts in motile fibroblasts. J Cell Biol 119, 1347-59.

Ren, X. D., Kiosses, W. B., Sieg, D. J., Otey, C. A., Schlaepfer, D. D. and Schwartz, M. A. (2000). Focal adhesion kinase suppresses Rho activity to promote focal adhesion turnover. Journal of Cell Science 113, 3673-8. 
Renshaw, M. W., Toksoz, D. and Schwartz, M. A. (1996). Involvement of the small GTPase rho in integrin-mediated activation of mitogen-activated protein kinase. Journal of Biological Chemistry 271, 21691-4.

Reuther, G. W., Lambert, Q. T., Booden, M. A., Wennerberg, K., Becknell, B., Marcucci, G., Sondek, J., Caligiuri, M. A. and Der, C. J. (2001). Leukemiaassociated Rho guanine nucleotide exchange factor, a Dbl family protein found mutated in leukemia, causes transformation by activation of RhoA. J Biol Chem 276, 27145-51.

Rid, R., Schiefermeier, N., Grigoriev, I., Small, J. V. and Kaverina, I. (2005). The last but not the least: the origin and significance of trailing adhesions in fibroblastic cells. Cell Motil Cytoskeleton 61, 161-71.

Ridley, A. J. (2001). Rho family proteins: coordinating cell responses. Trends Cell Biol 11, 471-7.

Ridley, A. J. and Hall, A. (1992). The small GTP-binding protein rho regulates the assembly of focal adhesions and actin stress fibers in response to growth factors. Cell 70, 389-99.

Ridley, A. J., Schwartz, M. A., Burridge, K., Firtel, R. A., Ginsberg, M. H., Borisy, G., Parsons, J. T. and Horwitz, A. R. (2003). Cell migration: integrating signals from front to back. Science $302,1704-9$.

Riento, K. and Ridley, A. J. (2003). Rocks: multifunctional kinases in cell behaviour. Nat Rev Mol Cell Biol 4, 446-56.

Rintoul, R. C. and Sethi, T. (2001). The role of extracellular matrix in smallcell lung cancer. Lancet Oncol 2, 437-42.

Rodriguez-Frade, J. M., Vila-Coro, A. J., Martin, A., Nieto, M., SanchezMadrid, F., Proudfoot, A. E., Wells, T. N., Martinez, A. C. and Mellado, M. (1999). 
Similarities and differences in RANTES- and (AOP)-RANTES-triggered signals: implications for chemotaxis. J Cell Biol 144, 755-65.

Rossman, K. L., Der, C. J. and Sondek, J. (2005). GEF means go: turning on RHO GTPases with guanine nucleotide-exchange factors. Nat Rev Mol Cell Biol 6, 167-80.

Rozengurt, E. (2007). Mitogenic signaling pathways induced by G proteincoupled receptors. J Cell Physiol 213, 589-602.

Rubinfeld, H. and Seger, R. (2005). The ERK cascade: a prototype of MAPK signaling. Mol Biotechnol 31, 151-74.

Sah, V. P., Seasholtz, T. M., Sagi, S. A. and Brown, J. H. (2000). The role of Rho in G protein-coupled receptor signal transduction. Annu Rev Pharmacol Toxicol 40, 459-89.

Samarin, S. N., Ivanov, A. I., Flatau, G., Parkos, C. A. and Nusrat, A. (2007). Rho/ROCK-II Signaling Mediates Disassembly of Epithelial Apical Junctions. Mol Biol Cell.

Sandilands, E., Cans, C., Fincham, V. J., Brunton, V. G., Mellor, H., Prendergast, G. C., Norman, J. C., Superti-Furga, G. and Frame, M. C. (2004). RhoB and actin polymerization coordinate Src activation with endosome-mediated delivery to the membrane. Dev Cell 7, 855-69.

Schaeffer, H. J. and Weber, M. J. (1999). Mitogen-activated protein kinases: specific messages from ubiquitous messengers. Mol Cell Biol 19, 2435-44.

Schafer, D. A., Welch, M. D., Machesky, L. M., Bridgman, P. C., Meyer, S. M. and Cooper, J. A. (1998). Visualization and molecular analysis of actin assembly in living cells. J Cell Biol 143, 1919-30. 
Schaller, M. D., Borgman, C. A., Cobb, B. S., Vines, R. R., Reynolds, A. B. and Parsons, J. T. (1992). pp125FAK a structurally distinctive protein-tyrosine kinase associated with focal adhesions. Proceedings of the National Academy of Sciences of the United States of America 89, 5192-6.

Schaller, M. D., Bouton, A. H., Flynn, D. C. and Parsons, J. T. (1993). Identification and characterization of novel substrates for protein tyrosine kinases. Progress in Nucleic Acid Research \& Molecular Biology 44, 205-27.

Schaller, M. D., Hildebrand, J. D., Shannon, J. D., Fox, J. W., Vines, R. R. and Parsons, J. T. (1994). Autophosphorylation of the focal adhesion kinase, pp125FAK, directs SH2-dependent binding of pp60src. Molecular \& Cellular Biology 14, 1680-8.

Schaller, M. D., Otey, C. A., Hildebrand, J. D. and Parsons, J. T. (1995). Focal adhesion kinase and paxillin bind to peptides mimicking beta integrin cytoplasmic domains. Journal of Cell Biology 130, 1181-7.

Schaller, M. D. and Parsons, J. T. (1995). pp125FAK-dependent tyrosine phosphorylation of paxillin creates a high-affinity binding site for Crk. Molecular \& Cellular Biology 15, 2635-45.

Schindler, T., Sicheri, F., Pico, A., Gazit, A., Levitzki, A. and Kuriyan, J. (1999). Crystal structure of Hck in complex with a Src family-selective tyrosine kinase inhibitor. Mol Cell 3, 639-48.

Schlaepfer, D. D., Broome, M. A. and Hunter, T. (1997). Fibronectinstimulated signaling from a focal adhesion kinase-c-Src complex: involvement of the Grb2, p130cas, and Nck adaptor proteins. Mol Cell Biol 17, 1702-13.

Schlaepfer, D. D., Hauck, C. R. and Sieg, D. J. (1999). Signaling through focal adhesion kinase. Progress in Biophysics \& Molecular Biology 71, 435-78. 
Schlaepfer, D. D., Jones, K. C. and Hunter, T. (1998). Multiple Grb2mediated integrin-stimulated signaling pathways to ERK2/mitogen-activated protein kinase: summation of both c-Src- and focal adhesion kinase-initiated tyrosine phosphorylation events. Mol Cell Biol 18, 2571-85.

Schober, M., Raghavan, S., Nikolova, M., Polak, L., Pasolli, H. A., Beggs, H. E., Reichardt, L. F. and Fuchs, E. (2007). Focal adhesion kinase modulates tension signaling to control actin and focal adhesion dynamics. J Cell Biol 176, 667-80.

Schwartz, M. (2004). Rho signalling at a glance. J Cell Sci 117, 5457-8.

Schwartz, M. A. (1997). Integrins, oncogenes, and anchorage independence. Journal of Cell Biology 139, 575-8.

Schwartz, M. A. (2001). Integrin signaling revisited. Trends in Cell Biology 11, 466-70.

Schwartz, M. A. and Ginsberg, M. H. (2002). Networks and crosstalk: integrin signalling spreads. Nature Cell Biology 4, E65-8.

Schwartz, M. A. and Ingber, D. E. (1994). Integrating with integrins. Molecular Biology of the Cell 5, 389-93.

Schwartz, M. A. and Lechene, C. (1992). Adhesion is required for protein kinase $\mathrm{C}$-dependent activation of the $\mathrm{Na}+/ \mathrm{H}+$ antiporter by platelet-derived growth factor. Proc Natl Acad Sci U S A 89, 6138-41.

Schwartz, M. A., Lechene, C. and Ingber, D. E. (1991). Insoluble fibronectin activates the $\mathrm{Na} / \mathrm{H}$ antiporter by clustering and immobilizing integrin alpha 5 beta 1, independent of cell shape. Proceedings of the National Academy of Sciences of the United States of America 88, 7849-53. 
Schwarzbauer, J. E., Tamkun, J. W., Lemischka, I. R. and Hynes, R. O. (1983). Three different fibronectin mRNAs arise by alternative splicing within the coding region. Cell $35,421-31$.

Sefton, B. M., Hunter, T., Beemon, K. and Eckhart, W. (1980). Evidence that the phosphorylation of tyrosine is essential for cellular transformation by Rous sarcoma virus. Cell 20, 807-16.

Seufferlein, T. and Rozengurt, E. (1994). Lysophosphatidic acid stimulates tyrosine phosphorylation of focal adhesion kinase, paxillin, and p130. Signaling pathways and cross-talk with platelet-derived growth factor. J Biol Chem 269, 9345-51.

Shattil, S. J. and Brugge, J. S. (1991). Protein tyrosine phosphorylation and the adhesive functions of platelets. Curr Opin Cell Biol 3, 869-79.

Sidani, M., Wessels, D., Mouneimne, G., Ghosh, M., Goswami, S., Sarmiento, C., Wang, W., Kuhl, S., El-Sibai, M., Backer, J. M. et al. (2007). Cofilin determines the migration behavior and turning frequency of metastatic cancer cells. J Cell Biol 179, 777-91.

Sieg, D. J., Hauck, C. R., Ilic, D., Klingbeil, C. K., Schaefer, E., Damsky, C. H. and Schlaepfer, D. D. (2000). FAK integrates growth-factor and integrin signals to promote cell migration. Nature Cell Biology 2, 249-56.

Slack-Davis, J. K., Eblen, S. T., Zecevic, M., Boerner, S. A., Tarcsafalvi, A., Diaz, H. B., Marshall, M. S., Weber, M. J., Parsons, J. T. and Catling, A. D. (2003a). PAK1 phosphorylation of MEK1 regulates fibronectin-stimulated MAPK activation. J Cell Biol 162, 281-91.

Slack-Davis, J. K., Eblen, S. T., Zecevic, M., Boerner, S. A., Tarcsafalvi, A., Diaz, H. B., Marshall, M. S., Weber, M. J., Parsons, J. T. and Catling, A. D. (2003b). 
PAK1 phosphorylation of MEK1 regulates fibronectin-stimulated MAPK activation. Journal of Cell Biology 162, 281-91.

Smilenov, L. B., Mikhailov, A., Pelham, R. J., Marcantonio, E. E. and Gundersen, G. G. (1999). Focal adhesion motility revealed in stationary fibroblasts. Science 286, 1172-4.

Sood, A. K., Coffin, J. E., Schneider, G. B., Fletcher, M. S., DeYoung, B. R., Gruman, L. M., Gershenson, D. M., Schaller, M. D. and Hendrix, M. J. (2004). Biological significance of focal adhesion kinase in ovarian cancer: role in migration and invasion. Am J Pathol 165, 1087-95.

Sood, A. K., Seftor, E. A., Fletcher, M. S., Gardner, L. M., Heidger, P. M., Buller, R. E., Seftor, R. E. and Hendrix, M. J. (2001). Molecular determinants of ovarian cancer plasticity. Am J Pathol 158, 1279-88.

Srinivasan, S., Wang, F., Glavas, S., Ott, A., Hofmann, F., Aktories, K., Kalman, D. and Bourne, H. R. (2003). Rac and Cdc42 play distinct roles in regulating $\mathrm{PI}(3,4,5) \mathrm{P} 3$ and polarity during neutrophil chemotaxis. J Cell Biol 160, 375-85.

Svitkina, T. M. and Borisy, G. G. (1999). Arp2/3 complex and actin depolymerizing factor/cofilin in dendritic organization and treadmilling of actin filament array in lamellipodia. J Cell Biol 145, 1009-26.

Thiery, J. P. (2002). Epithelial-mesenchymal transitions in tumour progression. Nat Rev Cancer 2, 442-54.

Tilghman, R. W., Slack-Davis, J. K., Sergina, N., Martin, K. H., Iwanicki, M., Hershey, E. D., Beggs, H. E., Reichardt, L. F. and Parsons, J. T. (2005). Focal adhesion kinase is required for the spatial organization of the leading edge in migrating cells. J Cell Sci 118, 2613-23. 
Togashi, H., Nagata, K., Takagishi, M., Saitoh, N. and Inagaki, M. (2000). Functions of a rho-specific guanine nucleotide exchange factor in neurite retraction. Possible role of a proline-rich motif of KIAA0380 in localization. $J$ Biol Chem 275, 29570-8.

Tosello-Trampont, A.-C., Nakada-Tsukui, K. and Ravichandran, K. S. (2003). Engulfment of Apoptotic Cells Is Negatively Regulated by Rho-mediated Signaling 10.1074/jbc.M306079200. J. Biol. Chem. 278, 49911-49919.

Totsukawa, G., Wu, Y., Sasaki, Y., Hartshorne, D. J., Yamakita, Y., Yamashiro, S. and Matsumura, F. (2004). Distinct roles of MLCK and ROCK in the regulation of membrane protrusions and focal adhesion dynamics during cell migration of fibroblasts. J Cell Biol 164, 427-39.

Toutant, M., Costa, A., Studler, J. M., Kadare, G., Carnaud, M. and Girault, J. A. (2002). Alternative splicing controls the mechanisms of FAK autophosphorylation. Mol Cell Biol 22, 7731-43.

Trogan, E., Feig, J. E., Dogan, S., Rothblat, G. H., Angeli, V., Tacke, F., Randolph, G. J. and Fisher, E. A. (2006). Gene expression changes in foam cells and the role of chemokine receptor CCR7 during atherosclerosis regression in ApoE-deficient mice. Proc Natl Acad Sci U S A 103, 3781-6.

Ullrich, A. and Schlessinger, J. (1990). Signal transduction by receptors with tyrosine kinase activity. Cell 61, 203-12.

van der Neut, R., Krimpenfort, P., Calafat, J., Niessen, C. M. and Sonnenberg, A. (1996). Epithelial detachment due to absence of hemidesmosomes in integrin beta 4 null mice. Nat Genet 13, 366-9.

Verkhovsky, A. B., Svitkina, T. M. and Borisy, G. G. (1999). Self-polarization and directional motility of cytoplasm. Curr Biol 9, 11-20. 
Verschueren, H., De Baetselier, P. and Bereiter-Hahn, J. (1991). Dynamic morphology of metastatic mouse T-lymphoma cells invading through monolayers of 10T1/2 cells. Cell Motil Cytoskeleton 20, 203-14.

Vicente-Manzanares, M., Webb, D. J. and Horwitz, A. R. (2005). Cell migration at a glance. J Cell Sci 118, 4917-9.

Vicente-Manzanares, M., Zareno, J., Whitmore, L., Choi, C. K. and Horwitz, A. F. (2007). Regulation of protrusion, adhesion dynamics, and polarity by myosins IIA and IIB in migrating cells. J Cell Biol 176, 573-80.

Wallar, B. J., Deward, A. D., Resau, J. H. and Alberts, A. S. (2007). RhoB and the mammalian Diaphanous-related formin mDia2 in endosome trafficking. Exp Cell Res 313, 560-71.

Wallingford, J. B., Rowning, B. A., Vogeli, K. M., Rothbacher, U., Fraser, S. E. and Harland, R. M. (2000). Dishevelled controls cell polarity during Xenopus gastrulation. Nature 405, 81-5.

Wang, F., Herzmark, P., Weiner, O. D., Srinivasan, S., Servant, G. and Bourne, H. R. (2002). Lipid products of $\mathrm{PI}(3) \mathrm{Ks}$ maintain persistent cell polarity and directed motility in neutrophils. Nat Cell Biol 4, 513-8.

Wang, Q., Liu, M., Kozasa, T., Rothestein, J. D., Sternweis, P. C. and Neubig, R. R. (2004). Ribozyme- and siRNA-mediated suppression of RGScontaining RhoGEF proteins. Methods Enzymol 389, 244-65.

Watterson, K. R., Lanning, D. A., Diegelmann, R. F. and Spiegel, S. (2007). Regulation of fibroblast functions by lysophospholipid mediators: potential roles in wound healing. Wound Repair Regen 15, 607-16. 
Webb, D. J., Donais, K., Whitmore, L. A., Thomas, S. M., Turner, C. E., Parsons, J. T. and Horwitz, A. F. (2004). FAK-Src signalling through paxillin, ERK and MLCK regulates adhesion disassembly. Nat Cell Biol 6, 154-61.

Webb, D. J., Parsons, J. T. and Horwitz, A. F. (2002). Adhesion assembly, disassembly and turnover in migrating cells -- over and over and over again. Nature Cell Biology 4, E97-100.

Webb, D. J., Zhang, H. and Horwitz, A. F. (2005). Cell migration: an overview. Methods Mol Biol 294, 3-11.

Weiner, T. M., Liu, E. T., Craven, R. J. and Cance, W. G. (1993). Expression of focal adhesion kinase gene and invasive cancer. Lancet 342, 1024-5.

Welch, M. D. and Mullins, R. D. (2002). Cellular control of actin nucleation. Annu Rev Cell Dev Biol 18, 247-88.

Wells, C. M., Walmsley, M., Ooi, S., Tybulewicz, V. and Ridley, A. J. (2004). Rac1-deficient macrophages exhibit defects in cell spreading and membrane ruffling but not migration. J Cell Sci 117, 1259-68.

Wierzbicka-Patynowski, I. and Schwarzbauer, J. E. (2003). The ins and outs of fibronectin matrix assembly. J Cell Sci 116, 3269-76.

Wilson, P. and Keller, R. (1991). Cell rearrangement during gastrulation of Xenopus: direct observation of cultured explants. Development 112, 289-300.

Wolf, K. and Friedl, P. (2006). Molecular mechanisms of cancer cell invasion and plasticity. Br J Dermatol 154 Suppl 1, 11-5.

Wolf, K., Mazo, I., Leung, H., Engelke, K., von Andrian, U. H., Deryugina, E. I., Strongin, A. Y., Brocker, E. B. and Friedl, P. (2003). Compensation mechanism in tumor cell migration: mesenchymal-amoeboid transition after blocking of pericellular proteolysis. J Cell Biol 160, 267-77. 
Wong, K., Van Keymeulen, A. and Bourne, H. R. (2007). PDZRhoGEF and myosin II localize RhoA activity to the back of polarizing neutrophil-like cells. J Cell Biol 179, 1141-8.

Wood, S., Jr. (1958). Pathogenesis of metastasis formation observed in vivo in the rabbit ear chamber. AMA Arch Pathol 66, 550-68.

Xiong, W. and Parsons, J. T. (1997). Induction of apoptosis after expression of PYK2, a tyrosine kinase structurally related to focal adhesion kinase. J Cell Biol $139,529-39$.

Xu, J., Wang, F., Van Keymeulen, A., Herzmark, P., Straight, A., Kelly, K., Takuwa, Y., Sugimoto, N., Mitchison, T. and Bourne, H. R. (2003). Divergent signals and cytoskeletal assemblies regulate self-organizing polarity in neutrophils. Cell 114, 201-14.

Yamada, T., Ohoka, Y., Kogo, M. and Inagaki, S. (2005). Physical and functional interactions of the lysophosphatidic acid receptors with PDZ domaincontaining Rho guanine nucleotide exchange factors (RhoGEFs). J Biol Chem 280, 19358-63.

Yang, J. T., Rayburn, H. and Hynes, R. O. (1993). Embryonic mesodermal defects in alpha 5 integrin-deficient mice. Development 119, 1093-105.

Yang, J. T., Rayburn, H. and Hynes, R. O. (1995). Cell adhesion events mediated by alpha 4 integrins are essential in placental and cardiac development. Development 121, 549-60.

Yeo, M. G., Partridge, M. A., Ezratty, E. J., Shen, Q., Gundersen, G. G. and Marcantonio, E. E. (2006). Src SH2 arginine 175 is required for cell motility: specific focal adhesion kinase targeting and focal adhesion assembly function. Mol Cell Biol 26, 4399-409. 
Yoneda, A., Multhaupt, H. A. and Couchman, J. R. (2005). The Rho kinases I and II regulate different aspects of myosin II activity. J Cell Biol 170, 443-53.

Yoneda, A., Ushakov, D., Multhaupt, H. A. and Couchman, J. R. (2007). Fibronectin matrix assembly requires distinct contributions from Rho kinases I and -II. Mol Biol Cell 18, 66-75.

Yron, I., Deckert, M., Reff, M. E., Munshi, A., Schwartz, M. A. and Altman, A. (1999). Integrin-dependent tyrosine phosphorylation and growth regulation by Vav. Cell Adhes Commun 7, 1-11.

Zaidel-Bar, R., Ballestrem, C., Kam, Z. and Geiger, B. (2003). Early molecular events in the assembly of matrix adhesions at the leading edge of migrating cells. J Cell Sci 116, 4605-13.

Zavortink, M., Bunch, T. A. and Brower, D. L. (1993). Functional properties of alternatively spliced forms of the Drosophila PS2 integrin alpha subunit. Cell Adhes Commun 1, 251-64.

Zhai, J., Lin, H., Nie, Z., Wu, J., Canete-Soler, R., Schlaepfer, W. W. and Schlaepfer, D. D. (2003). Direct interaction of focal adhesion kinase with p190RhoGEF. J Biol Chem 278, 24865-73.

Zheng, Y., Hart, M. J. and Cerione, R. A. (1995a). Guanine nucleotide exchange catalyzed by dbl oncogene product. Methods Enzymol 256, 77-84.

Zheng, Y., Olson, M. F., Hall, A., Cerione, R. A. and Toksoz, D. (1995b). Direct involvement of the small GTP-binding protein Rho in lbc oncogene function. J Biol Chem 270, 9031-4.

Zucker, S., Cao, J. and Chen, W. T. (2000). Critical appraisal of the use of matrix metalloproteinase inhibitors in cancer treatment. Oncogene 19, 6642-50. 
